\title{
ACNP 60th Annual Meeting: Author Index
}

\author{
Neuropsychopharmacology (2021) 46:519-553; https://doi.org/10.1038/s41386-021-01239-4
}

Last Name, First Name

Aaronson, Scott T.

Abbasi, Fahim

Abbott, Christopher

Abdelmesih, Brenda

Abdoli, Parmida

Abdul-Waalee, Halimah

Abelson, James

Abend, Rany

Abi-Dargham, Anissa

Abraham, Antony

Abram, Samantha

Abu Suhaiban, Hiba ................................................................................P130

Aceto, Giuseppe ......................................................................................... P308

Acevedo, Elia .................................................................................................P272

Acharya, Nimish ........................................................................................P530

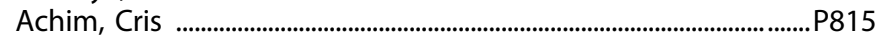

Acion, Laura ..............................................................................................P184

Adams, Faith ............................................................................................P164

Adams, Gordon ........................................................................................ P4

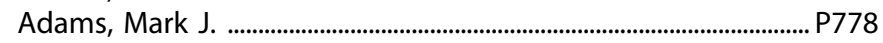

Adank, Danielle .......................................................................................P629

Adcock, R. Alison ..................................................................................... P122

Addington, Jean ..................................................................................... P545

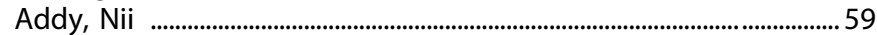

Adebimpe, Azeez ...................................................................... P148, P214

Adeojo, Lilian .........................................................................................P273

Adermark, Louise ............................................................................................. P36

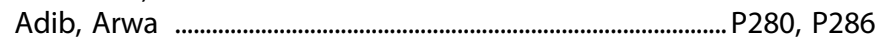

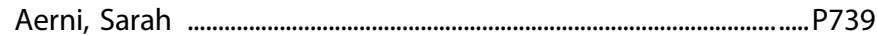

Agarwal, Khushbu ............................................................................P680

Agarwal, Sri Mahavir ......................................................P453, P509, P559

Aggarwal, Nakul .......................................................................................... P85

Aggensteiner, Pascal ............................................................................... P447

Aghera, Veronica ..............................................................P397, P398, P399

Ago, Yukio ...................................................................................................P553

Agrawal, Arpana ........................................................................................ P184

Agrawal, Pankaj .................................................................................... P512

Ahmari, Susanne ...............................................................50, P438, P446

Ahmed, Ryan ........................................................................................P368

Ahmed, Sanjana ................................................................................................... P342

Ahn, Hyeon Min ............................................................................. 251, P357

Ahn, Woo-Young ….................................................................................... P132

Aizenstein, Howard ..................................................... P11, P32, P74, P825

Aizley, Harlyn ............................................................................................P326

Ajam Oughli, Hanadi .......................................................................... P231

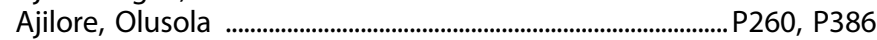

Akbarian, Schahram …………........................................461, P744, P808

Akdilek, Naz ..............................................................................................P635

Aki, Tsuchiyagaito ................................................................................... P145

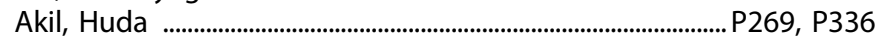

Akula, Nirmala .......................................................................................... P123
Al Jurdi, Rayan .......................................................................................... P328

Alam, M. Ashad ............................................................................................ P164

Alan, Davis ..................................................................................................P353

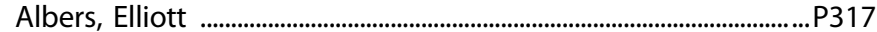

Albert, Kimberly ................................................................................................... P368

Albert, Umberto ...................................................................................... P440

Albertella, Lucy …….............................................................................. 1.4

Albott, Cristina ….................................................................................... P247

Al-Chalabi, Nzaar ........................................................................................P479

Alegria, Margarita ....................................................................................................... 12

Alexander, Desi ............................................................................................. P664

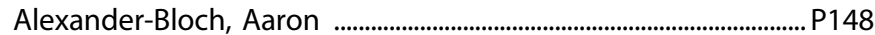

Aleyasin, Hossein ....................................................................................... P657

Alganem, Khaled ..............................................................P322, P550, P559

Alghorazi, Rizk ................................................................................................. P39

Alia-Klein, Nelly .............................................................................P625, P752

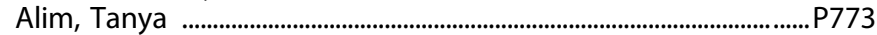

Alkelai, Anna .......................................................................................................P566

Allen, John ....................................................................................................P563

Allen, Madeleine ..............................................................................P106

Allen, Stephen ........................................................................................... P769

Allerup, Peter ..................................................................................................P524

Allocca, Giancarlo .................................................................................... P613

Alloo, Kumayl ..............................................................................................P265

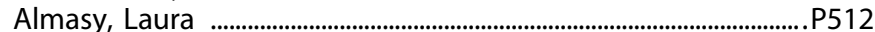

Almeida, Ana .............................................................................................P739

Almeida, Jorge ........................................................................................... P332

Almeida, Osvaldo ............................................................................................ P17

Almira, Erika ...............................................................................................P285

Alnafisah, Rawan ...................................................................................P322

Alonso Caraballo, Yanaira ............................................................................. P62

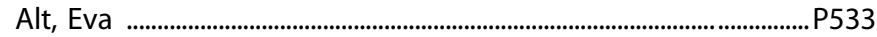

Altemus, Megan ................................................................................................. P40

Alvarado-Torres, John ..............................................................................P656

Alvarez, Emmanuel ...............................................................................P738

Alvarez, Kelvin ................................................................................................. P68

Alvino, Filomena ......................................................................................... P570

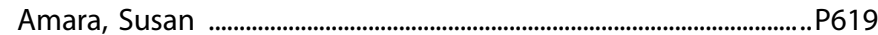

Amaral, David ............................................................................... P165, P543

Amat, Jose ........................................................................................................P266

Ambrosen, Karen ........................................................................................ P542

Ameis, Stephanie H. ................................................................................ P418

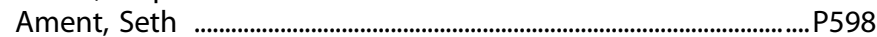

Amico, Kristen .................................................................................................. P2 263

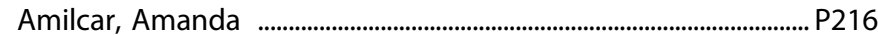

An, Xiaoxian .....................................................................................................P327

Anacker, Christoph ..................................................................... 24, P346

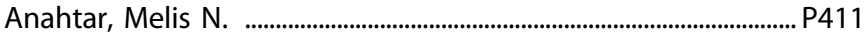

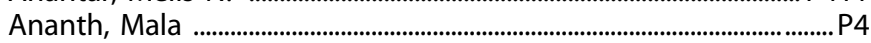

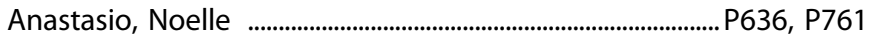

Andari, Elissar ........................................................................................P112

Andersen, Helle ..................................................................................... P542 
Anderson, Alicia

P773

Anderson, Ashley

Anderson, Ellen

Anderson, Ethan

P245, P246

P133

P618

P212

P751

P494

Anderson, Tanner

P32, P74

Andreescu, Carmen

... P47

Andrews, Olivia ................................................................................ P536

Andryskova, Lenka ............................................................................. P139

Ang, Gavrila

P29

Angarita-Africano, Gustavo

P592, P668

Angelis, Ariana

Angstadt, Mike

P717

Ansorge, Mark

P801

P292

P307

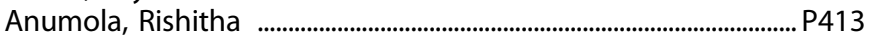

Aponte, Yeka ...............................................................................................30.2

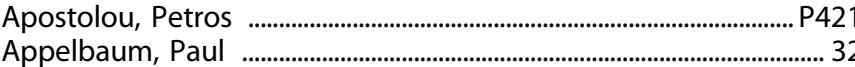

Apseloff, Glen ........................................................................................P315

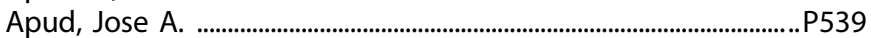

Aquino-Miranda, Guillermo ..............................................................P745

Arango, Victoria

P259, P264

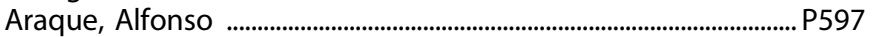

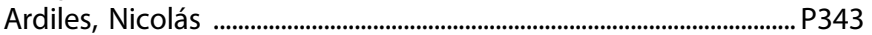

Arey, Rachel ....................................................................................................... P15

Arguello, Amy A. ................................................................................... P768

Argyelan, Miklos ……………………………........P374, P469, P506, P810

Arias, Albert ..........................................................................................................P630

Arjavalingam, Anusuya .........................................................................P401

Armenta-Resendiz, Monserrat ........................................................P712

Arnold, Miranda ........................................................................................ P263

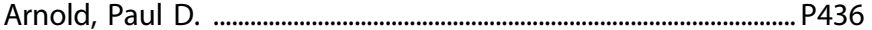

Arnold, Steven E. ............................................................................... P4 11

Arnsten, Amy ....................................................................................................... 6.4

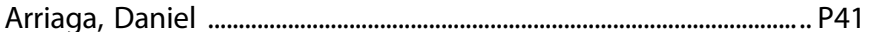

Arruda-Carvalho, Maithe ...................................................................P166

Arruda-Neto, Eurico ............................................................................P424

Arslan, Ahmed ....................................................................................... P735

Artin, Hewa ............................................................................................ P83

Arulpragasam, Amanda ………………………………………………..... P310

Asano, Satoshi ........................................................................................... P553

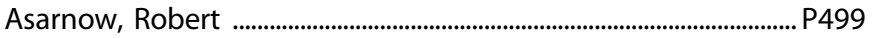

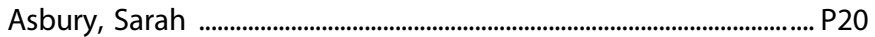

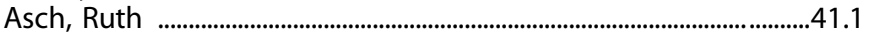

Asgariroozbehani, Roshanak ...........................................P509, P559

Asgharnejad, Mahnaz ................................................P462, P514, P560

Ash, Ryan ...............................................................................................P811

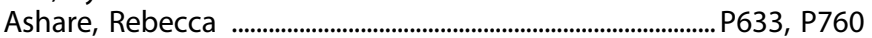

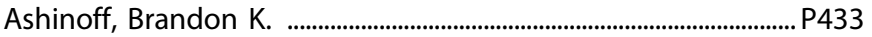

Ashraf, Aseelah ...................................................................................... P57

Ashworth, Judy ........................................................................... 2225, P315

Askins, Laura ……………………….......................................................P8

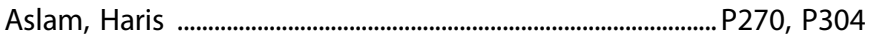

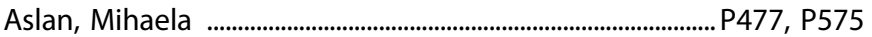

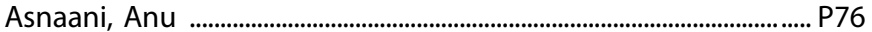

Assali, Ahlem ...........................................................................................P712

Aston-Jones, Gary 20, ........................................................................P659

Athreya, Arjun ............................................................................................. P349

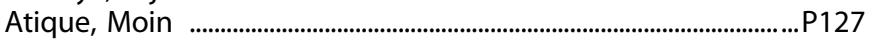

Atkinson, Elizabeth .............................................................................P736

Atkinson, Sarah ……………………………………………………………... P28

Atlas, Lauren .................................................................................................. P43

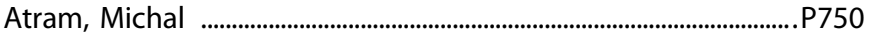

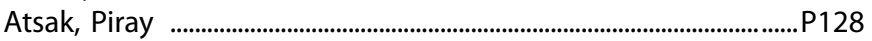

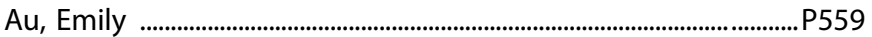

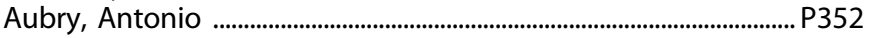

Auerbach, Randy ............................................................................... P325

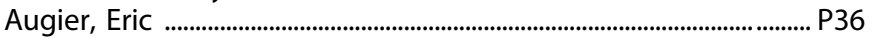

Augier, Gaëlle ........................................................................................... P36

Augimeri, Leena .......................................................................................... P156

Augustin, Shana ......................................................................................... P413

Aupperle, Robin ..........................................................51, P145, P287

Autry, Anita ............................................................................................P212

Auvil, Daley ...............................................................................................P549

Avelar, Alicia ....................................................................................................P677

Averbeck, Bruno .................................................................................... P403

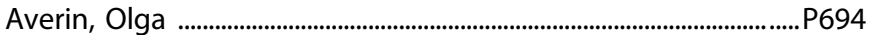

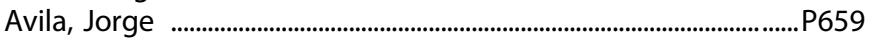

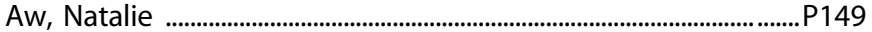

Awad, Angelina ………………………………………………………... P467

Axelowitz, Jared .............................................................P678, P680, P700

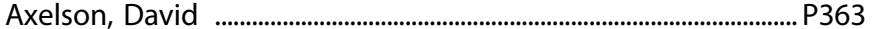

Ayappa, Indu ..............................................................................................P409

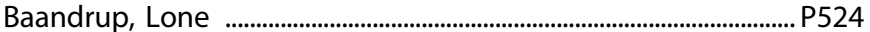

Babadi, Baktash ……………………...................................................... P816

Babb, James ................................................................................................. P19

Bachi, Keren ..................................................................P674, P742, P807

Bachman, Peter M. ................................................................................. P545

Back, Sudie .................................................................................................17.1

Backenroth, Daniel .................................................................................. P540

Baez-Nieto, David ................................................................................... P472

Bagley, Jared ....................................................................... P199, P714

Bagot, Rosemary ........................................................................... P295, P347

Bahji, Anees ..............................................................................................P382

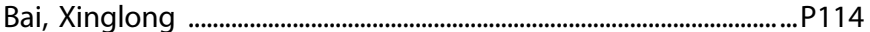

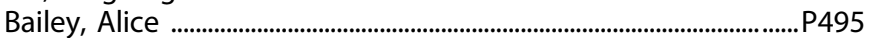

Bailey, Lauren .......................................................................................P199

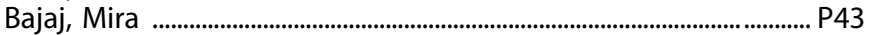

Bajo, Michal .............................................................................................P681

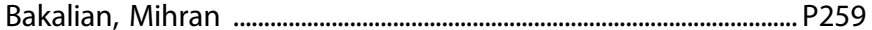

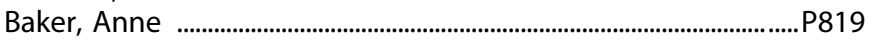

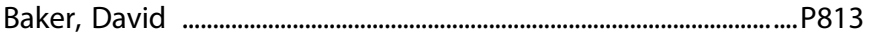

Baker, Dewleen ................................................................................................ P83

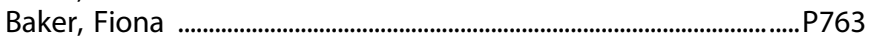

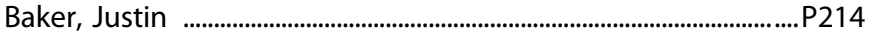

Baker, Matthew ......................................................................................... P754

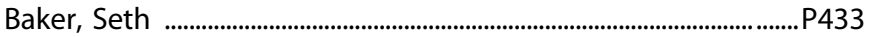

Bal, Aneesh ........................................................................................................P267

Balana, Bartosz ....................................................................................... P330

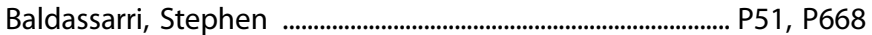

Balderston, Nicholas ......................................................................... P323, P392

Baldi, Pierre .................................................................................................... P3

Bale, Tracy ................................................................... P136

Ballard, Elizabeth ........................................................................................... P402

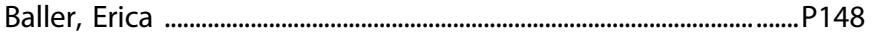

Balsam, Peter .........................................................128, P207, P446, P534

Balu, Darrick ....................................................................................................P47

Banasr, Mounira ............................................................................................. P344

Banavar, Nidhi ............................................................................................... P58

Bangasser, Debra ................................................. 10.2, P294, P684, P737

Banihashemi, Layla ........................................................................................ P825

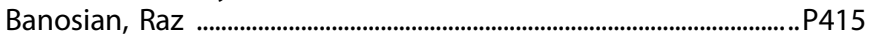

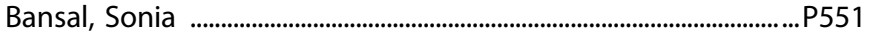

Baptiste, Ian ..............................................................................................P505

Barak, Lawrence .................................................................................... P596

Barak, Segev ................................................................................................P205

Baram, Tallie Z. ....................................................... 54.1, P114, P387, P665

Baratta, Michael ............................................................................P266

Barba-Villalobos, Griselda ................................................................................. P127

Barbee, Britton ........................................................................................ P663

Barber, Anita ………………………………………......... P455, P506

Barbier, Estelle ........................................................................... P36, P711 
Barbieri, Riccardo ................................................................................................ P326

Barbon, Alessandro

Barbu, Miruna

.P778

Barch, Deanna ....

Barchiesi, Riccardo

P28, P135, P168

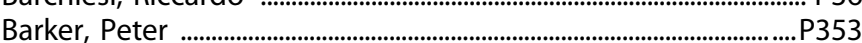

Barko, Kelly ............................................................................................P360

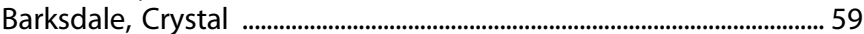

Barnett, lan .........................................................................................................P214

Barnhill, Olivia .................................................................................................. P244

Barr, Peter .......................................................................................... P575, P703

Barredo, Jennifer ................................................................................. P812

Barrett, Frederick .................................................................... P353, P784

Barrientos, Ruth ...................................................................................... P16

Barry, Alida ..........................................................................................P518

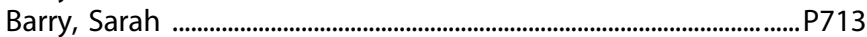

Bartlett, Andrew ...................................................................... P278, P379

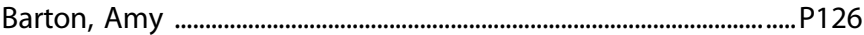

Bartova, Lucie ............................................................................................P240

Barzilay, Ran ..........................................................................................P131

Basavanhalli, Samhita ............................................................................ P89

Basgoze, Zeynep .......................................................................................... P817

Baskerville, Wave-Ananda ..................................................................P685

Bassett, Danielle .......................................................................................... P214

Bastos, Georgia ........................................................................................ P464

Batah, Sabrina ............................................................................................... P424

Bates, Emily .................................................................................................P704

Batistuzzo, Marcelo .............................................................................P444

Batra, Kanha ........................................................................ P27, P267, P672

Bauer, Clemens ......................................................................................... P467

Bauer, James .........................................................................................P529

Bauer, Lance .....................................................................................................P184

Bauman, Melissa ................................................................... P165, P543

Baumann, Michael ...................................................................................P732

Baumeister, Sarah ................................................................................. P447

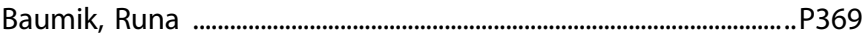

Bautista, Malia ........................................................................................... P623

Bavley, Charlotte ..............................................................P294, P684, P737

Bayat, Anita ....................................................................................P108

Bazán, Paulo .............................................................................................P444

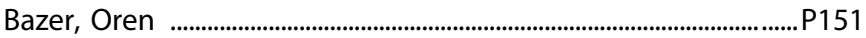

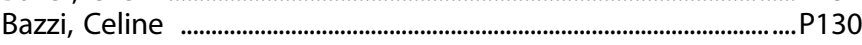

Bean, Erin ..................................................................................................... P89

Bearden, Carrie E ........................................................P499, P545, P570

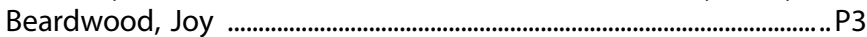

Beaulieu, Jean-Martin ..............................................................................P217

Beaumont, Kristin ............................................................................... P194, P601

Beausoleil, Nancy ..................................................................................P206

Bebko, Genna _....................................................................... P270, P304

Becerra, Patricia ................................................................................... P123

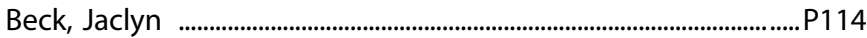

Becker, Howard ..........................................................................P618, P698

Becker, Jill .................................................................................10, 10.3

Becker, Kendra .................................................................................. P186

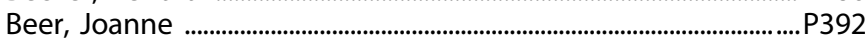

Beermann, Adam .................................................................................. P475

Beggs, Alan ...............................................................................................P512

Begni, Veronica ..........................................................................P316, P320

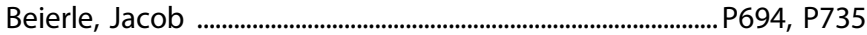

Beierlein, Jennifer .......................................................................................... P429

Bekinschtein, Pedro ................................................................................. P802

Belelli, Delia .......................................................................................................P114

Belfort, Gabriel ....................................................................................... P2 235

Belger, Aysenil ............................................................................................ P545

Belilos, Andrew .............................................................................................. P52

Bell, Richard ........................................................................................P774

Belleau, Emily ...................................................................................................P389

Bellesheim, Katie ........................................................................................... P1 26
Belmonte da Silveira, Bárbara Luiza ..............................................P675

Belov, Vasily ..............................................................................................P500

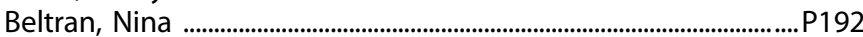

Beltz, Adriene M. ................................................................................ P140

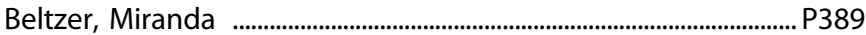

Ben, Siemsen ............................................................................................P641

Bender, Brooke ........................................................................................... P643

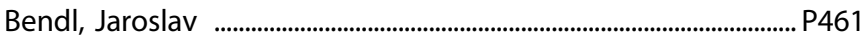

Benn, Emma ....................................................................................................... 12

Benoit, Laura ................................................................................................P537

Benson, Deanna ........................................................................................... P2 265

Bentley, Sean ............................................................................................ P83

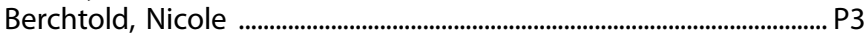

Berenson, Charles ................................................................................. P334

Berg, Hannah ......................................................................................P716

Berger, Shelley ................................................................................................. P664

Bergink, Veerle ............................................................................................. P178

Bergman, Jack ...................................................................................P653

Bergman-Levy, Tal ................................................................................... P544

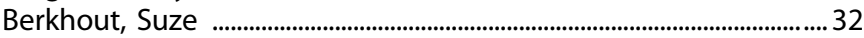

Berlow, Yosef ..........................................................................................P250

Berman, Karen .......................................... P107, P147, P450, P478, P539

Berman, Steven ..............................................................................................

Bermingham, Mairead .................................................................................... P778

Bernat, Edward ........................................................................................ P669

Berner, Laura ......................................................................................................14.

Bernhardt, Elisabeth ............................................................................ P406

Bernstein, Hans-Gert ............................................................................ P577

Berretta, Sabina ................................................................................... P93, P236

Berretta, Sarah .................................................................................................. P496

Berrettini, Wade ………………………………………………………….... P339

Berry, Jack ................................................................................................. P50

Berry, Jarett .................................................................................................

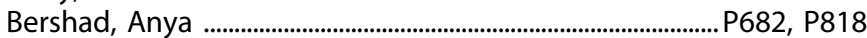

Berta, Rachel ........................................................................................... P32

Bertelsen, Kirk .........................................................................................P311

Bertocci, Michele ..............................................................270, P284, P304

Bertz, Jeremiah .........................................................................................P728

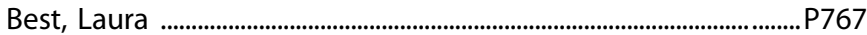

Betzhold, Sara .....................................................................................P640

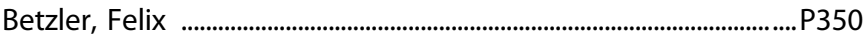

Beunders, Alexandra ................................................................................... P17

Beversdorf, David ................................................................................... P126

Beyeler, Anna ...............................................................................................P267

Bhakta, Savita .....................................................................................P562

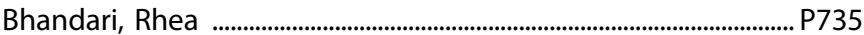

Bhanu, Natarajan ................................................................................................ P4

Bharadwaj, Rahul ................................................................................... P93

Bhatt, Shivani .......................................................................... P58, P783

Bhavna, Solanki ......................................................................................... P28

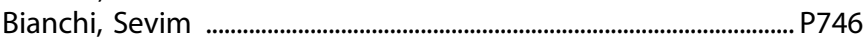

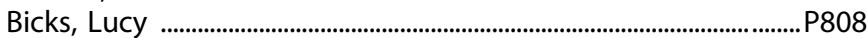

Bidwell, L. Cinnamon .............................................................................P616

Bierlein-De La Rosa, Gabrielle ........................................................P161

Biernacka, Joanna ........................................................................... P349, P381

Biesecker, Catherine ................................................................P699, P709

Biesecker, Erin ........................................................................................... P679

Bigdeli, Tim

P226, P477, P573, P575

Biggerstaff, Natalie ..............................................................................P161

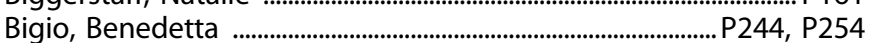

Bilbo, Staci ....................................................................... 129, P345, P416

Bilder, Robert .................................................................................................P695

Bilotti, Michelle ........................................................................................... P764

Birmaher, Boris ….........................................................2284, P304, P363

Birnbaum, Michael ......................................................................... P455, P506

Birnbaum, Rebecca ............................................................................. P521

Birnie, Matthew ………………………………………………….... P387, P665

Bishop, James ..........................................................................................P176 
Bishop, Jeffrey

Bishop, Ruth

P333

Bismark, Andrew

Bittencourt, Augusto Martins Lucas

Bizon, Jennifer

Bjork, James

Black, Samantha

Blackford, Jennifer

Blackman, Rachael

Blaes, Shelby

Blake, Kyomi

Blankenship, Kayla

Blass, Benjamin E

Blay, Christopher

Blaze, Jennifer

Blazer, Annie

Blennow, Kaj .

Blessing, Esther

Blier, Pierre

Bliss-Moreau, Eliza

Blough, Bruce

Blumberg, Hilary

Blumberger, Daniel M.

Boateng, Comfort

Bobadilla, Ana-Clara

Boberg, Julia

Bobo, William ...

Bocarsly, Miriam

Bodinayake, Kasuni

Bodurka, Jerzy

Boehme, Rebecca

Bogachuk, Alina

Bøgehave, Hjalte

Boggs, Anna

Bohnsack, John Peyton

Bohon, Cara

Boileau, Isabelle

Boivin, Michel

Bojesen, Kirsten Borup

Bolanos-Guzman, Carlos

Bolden, Khalima

Boldizsar, Noelia

Boldizsar, Noey

Boldrini, Maura

Bolen, Joe

Boley, Angela ...

Bolomope, Janet

Bolshakov, Vadim

Bolton, Jessica

Bolton, Thomas

Bomyea, Jessica

Bonagura, Darla

Bonanno, Giambattista

Bonar, Erin

Bonar, Lisa

Bonaventura, Jordi

Bonaventure, Pascal

Bondy, Emma

Bongiovanni, Angela

Bonifacino, Tiziana

Bonilla, Hector

Bonner, Daniel

Book, Sarah

Boomhower, Brent

Bora, Esha

Bordt, Evan

Borg, Breanna

Borio, Matilde
P83

P675

P410, P640

P693

P689

P104, P169, P486

P450

P640

P265

P712

P770

P555

P744

P568

P306

P409

P367, P376

58

P192

P17, P364

P232, P314, P418, P497

P590

P628, P772

P445

..P349

P191

P642

P145

P771

... P90

P542

P173

P747

P193

P425, P767

P153

P526, P542

P249, P301

P555

P547

P590

P259, P264

P415

P489

P773

P195, P621

P114, P345, P665

P389

P88

P738

.. P87

P748

P304

$\mathrm{P} 175, \mathrm{P} 233$

P330, P423, P617

P781

P684

P87

P253

P415

P639

P677, P701

P745

P416

P111

P267
Borkar, Chandrashekhar P89

Bortz, David

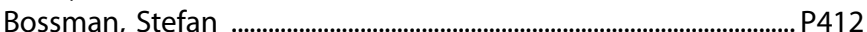

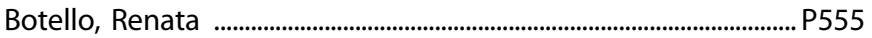

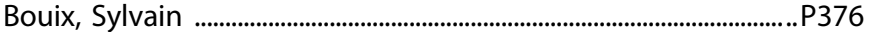

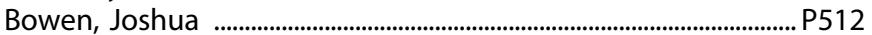

Bowie, Christopher .................................................................................... P314

Bowman, Melodi A. ..............................................................................P671

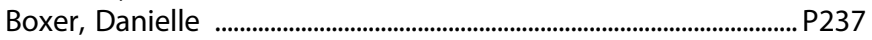

Boyle, Sara ....................................................................................................... P73

Boyt, Kristen .....................................................................................................P683

Bradberry, Charles W. .......................................................................... P729

Bradford, Daniel ……………………………………………........... P31

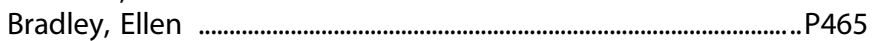

Brady, Kathleen . . . ................... 7

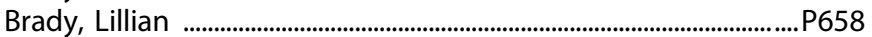

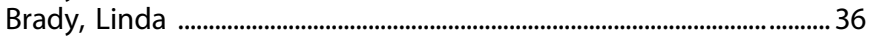

Brady, Rebecca ……………………….................................................. P168

Brady, Roscoe ............................................................................................P4

Braff, David ...................................................................................P477, P573

Braide, Biobele ....................................................................................................P98

Brain, Cecilia ..................................................................................................P490

Branda, John ......................................................................................P411

Brandão, Marcus .................................................................................... P444

Brandeis, Daniel ...................................................................................... P447

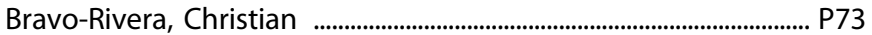

Brawer, Jacob .............................................................................................P253

Brazdil, Milan ..............................................................................................P139

Breier, Alan ......................................................................................................P565

Breithaupt, Lauren .................................................................................. P186

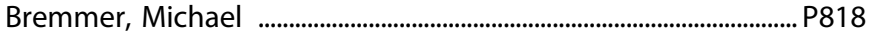

Brendgen, Mara ................................................................................................. P153

Brenhouse, Heather ...............................................................................P157

Brennan, Brian ....................................................................................................... P183

Brennan, Molly ........................................................................................... P677

Brenner, Helena .................................................................................. P307

Bretton, Zachary ………………………………………………………......... P128

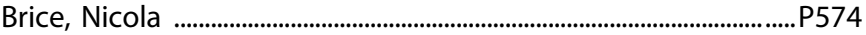

Brickman, Adam ....................................................................................49

Bridi, Morgan .................................................................................................... 136

Briggs, Farren .................................................................................................... P17

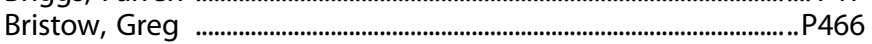

Britton, Jennifer ................................................................................... P172

Brock, Ashley ............................................................................................P713

Brockmeier, Austin ................................................................................... P474

Brodsky, Jeffery ............................................................................................. P536

Brody, Arthur .........................................................................................................P815

Broeders, Tommy .................................................................................... P218

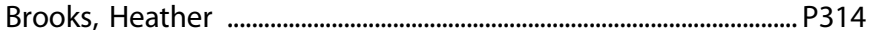

Brosseau, Pat .............................................................................................P282

Brotman, Melissa ............................................ 47, P37, P214, P403, P799

Brouillard, Alexandra ........................................................................................ P81

Brown, Caleb ..............................................................................................P221

Brown, Catherine ............................................................................P826

Brown, Colville .......................................................2222, P223, P224, P271

Brown, Eric ........................................................................................P579

Brown, Jadeda ........................................................................................ P338

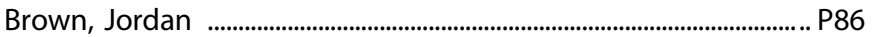

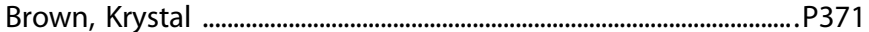

Brown, Larry ...............................................................................................P206

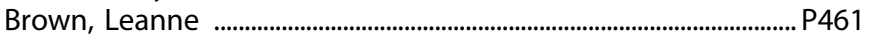

Brown, Lily .................................................................................................................... P76

Brown, Lisa ....................................................................................................P334

Brown, Oneysha .................................................................................... P230

Brown, Sandra .............................................................................................. P763

Brown, Sarah ..............................................................................................P618

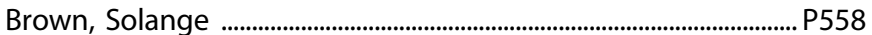

Brown, Stephanie ................................................................................... P471 
Browne, Caleb

P301, P744

Browne, Caroline

P366

Browning, Brittney

P726

Brownstein, Catherine A

P512

Brucar, Leyla

P595

Bruce, Heather

P456, P586

Bruchas, Michael

P337, P600

Brumback, Ty

P763

Bruno, Davide

.P306

Bruno, Jennifer

P105, P393

Brunoni, Andre P803

Brunst, Kelly

44.2

Brusman, Liza

P809

Bryan, Angela

P616

Bryant, Camron

P694, P735

Buban, Katelyn

P420, P432

Buccini, Michelle

P683

Buchanan, Robert W.

P469, P487

Buchhalter, August

P315

Bucholz, Kathleen

P184, P204

Buck, Benjamin

P465

Buck, Silas

P427

Buck, Tristram

P466

Bugarski-Kirola, Dragana

P490, P518

Bulik, Cynthia

P445

Burch, Ronald

P311

Burdette, Evan

P172

Burdick, Katherine

P228, P298

Burgdorf, Jeffrey

P311

Burgos-Robles, Anthony ........................................................................... P41

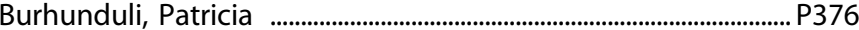

Burk, Diana ...................................................................................................P403

Burkauskas, Julius .............................................................................. P434

Burkett, James ....................................................................................... P142

burstein, Ethan ...................................................................................... P480

Busler, Jessica ………………………..........................P251, P385, P820

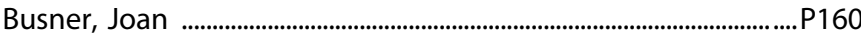

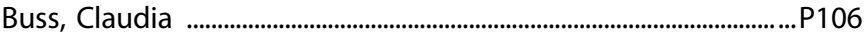

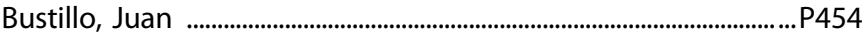

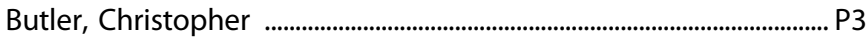

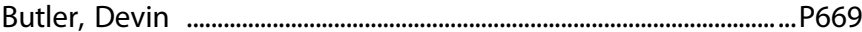

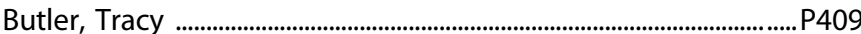

Butman, John ................................................................................ P699, P709

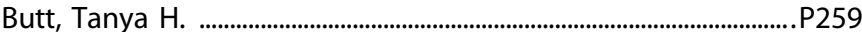

Butts Pauly, Kim ................................................................................... P811

Buxbaum, Joseph ....................................................................................... P445

Byrnes, Julie ................................................................................................P674

Byron, Brooke ........................................................................................P476

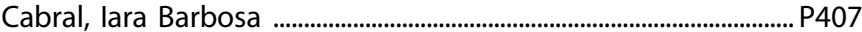

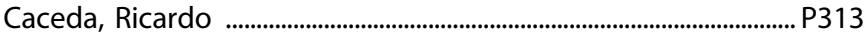

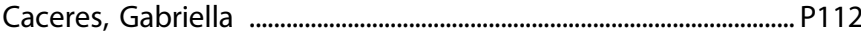

Cadenhead, Kristin S ...........................................................................P545

Cahalan, Michael .................................................................................. P114

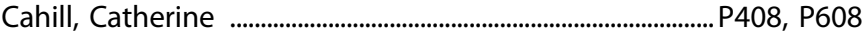

Cahill, Kelly ........................................................................................................P532

Cai, Denise .................................................................................. P84, P744

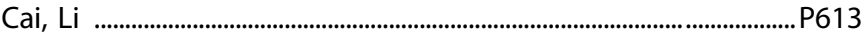

Cai, Min ........................................................................................................P177

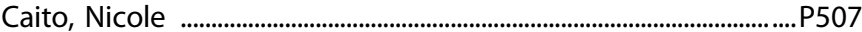

Calabro, Finnegan ...................................................................................... P568

Calakos, Katina ......................................................................................... P668

Calakos, Nicole …………………………………………………………......P609

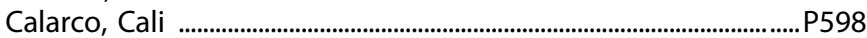

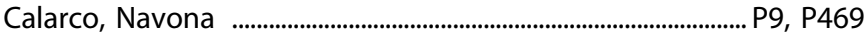

Calarge, Chadi ................................................................................................... P127

Calhoon, Gwendolyn ...............................................................................P267

Caligiuri, Stephanie ...................................... P194

Calipari, Erin

P177, P417, P629, P655, P658, P740, P743
Calkins, Monica

P214, P571

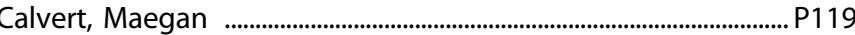

Camchong, Jazmin ................................................................................P605

Cameron, Judy ............................................................................................ P121

Cameron, Lindsay ....................................................................................... 15

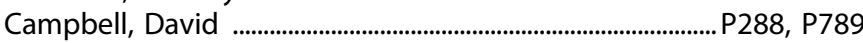

Campos, Alline ....................................................................................... P424

Camprodon, Joan ............................................................................ P280

Campus, Paolo ........................................................................................ P626

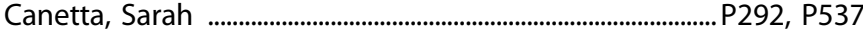

Cannon, Tyrone .............................................................................P545, P695

Cantos, Adriana ...................................................................................... P305

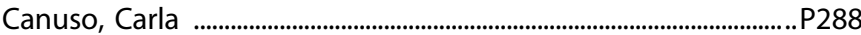

Cappi, Carolina ............................................................................................ P146

Caradonna, Salvatore .......................................................................... P269

Caravaggio, Fernando ............................................................................P579

Carbajal, Jessica ........................................................................................... P313

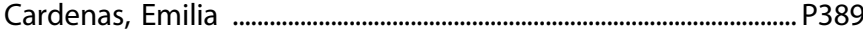

Cardinal, Rudolf ........................................................................................... P800

Cardinale, Elise ..................................................................................................P37

Cardona, Astrid ..................................................................................... P249

Carhart-Harris, Robin ............................................................................... P800

Carillo, Angelica ...................................................................................... P278

Carlezon, William ............................................................... P35, P278, P379

Carlson, Erika ........................................................................................P731

Carlson, Marina ................................................................................................. P171

Carlson, Marlene ...........................................................................P525, P574

Carlton, Mark ..................................................................................................P574

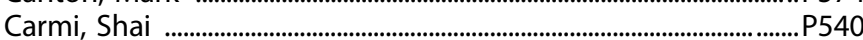

Carmody, Thomas ....................................................................................... P349

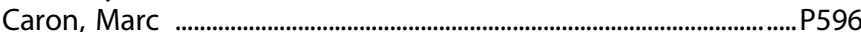

Carpenter, Marco ............................................................................................. P756

Carpenter, William ..................................................................... 19, 32, 37

Carr, Meagan ..........................................................................................P804

Carraco, Megan ....................................................................P678, P680, P700

Carrette, Lieselot ..........................................................................P677, P701

Carrington, Holly .................................................................................... P186

Carrion, Ricardo E .................................................................................... P545

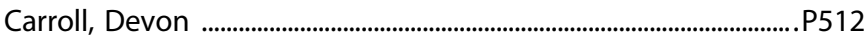

Carroll, Kathleen ....................................................................................... P749

Carson, Richard ..................................................... P58, P210, P592, P783

Carta, Kayla ..................................................................................... P312, P393

Carter, Cameron ...................................................P165, P457, P543, P555

Carter, Sierra ............................................................................................27.3

Casello, Sanne ......................................................................................... P779

Cassano, Paolo ………………………...................................... P280, P286

Cassidy, Clifford ...........................................................................................P9, P10

Casteeen, Emily ........................................................................................... P31

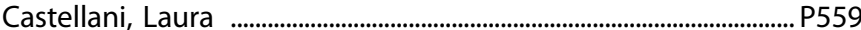

Castellanos Ryan, Natalie .................................................................. P153

Castillo, Monica Feliz ................................................................. P29, P305

Castillo, Pablo E. ........................................................................... P293

Castro, Daniel .................................................................................. 42.3, P600

Catallini, Joseph L. ...........................................................................P539

Caterina, Oneto ……………………………………………………….... P334

Cathomas, Flurin ...............................................P230, P301, P342, P352

Cattaneo, Annamaria ............................................................P316, P320

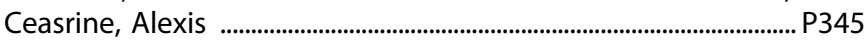

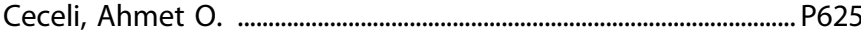

Cecil, Kim .............................................................................................P399

Centanni, Samuel ........................................................................... P86, P601

Cerceo Page, Stephanie .......................................................................... P539

Cernasov, Paul ......................................................................................P388, P789

Cervera Juanes, Rita ....................................................................... P794

Cerwensky, Allison ...................................................................................... P739

Cetin-Karayumak, Suheyla .......................................................P487, P565

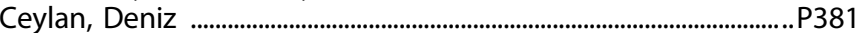

Cha, Emily ................................................................................................P295 
Cha, Jiook

P7, P132, P299

Chahine, Britton

Chamberlain, Samuel

Chamoun, Mira

Chan, Grace

$1,1.2, \mathrm{P} 434, \mathrm{P} 443$

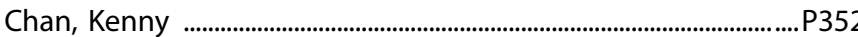

Chan, Nathan ..................................................................................P579

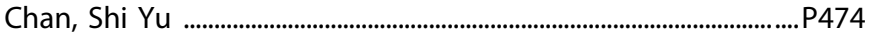

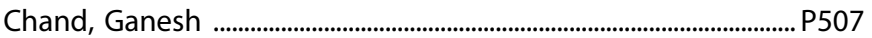

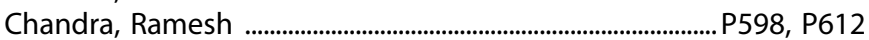

Chang, Bernard ........................................................................................... P99

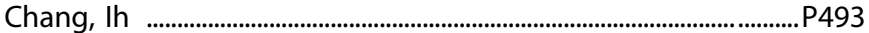

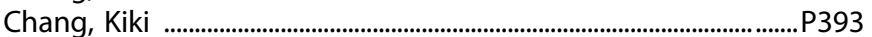

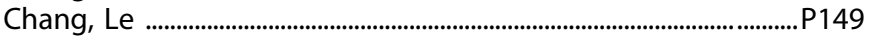

Chang, Lee ........................................................................................................P328

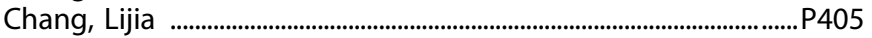

Chang, Victoria ........................................................................................... P602

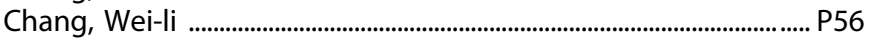

Chanthongdee, Kanat ......................................................................... P36

Chao, Linda ...................................................................................................... P42

Chapman, Angela ................................................................................. P562

Charlet, Katrin .........................................................................................P778

Charney, Alexander ...................................................................................... P62

Charney, Dennis ........................................................................................... P68

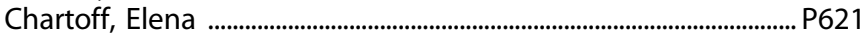

Chase, Henry

P270, P304

Chatzinakos, Christos

P91, P93, P290

Chávez, Andrés E.

Chavkin, Charles

P343

P779

Chawla, Sumedha

Cheetham, Claire

P678, P680, P700

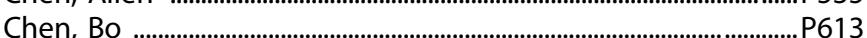

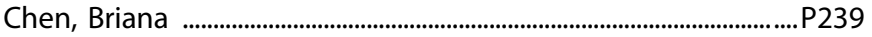

Chen, Cathy ……….................................................................................P324

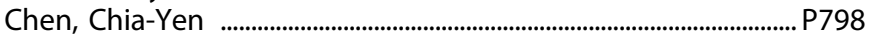

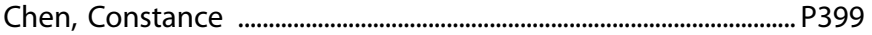

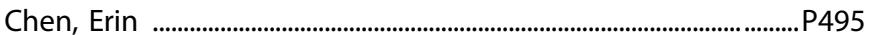

Chen, Eunice ..................................................................................... 116

Chen, Haiying ............................................................................................P308

Chen, Hannah S. ............................................................................................ P27

Chen, Huei-Ying ........................................................................................ P539

Chen, Jianhua ….................................................................................P396

Chen, Jingyun ............................................................................................. P19

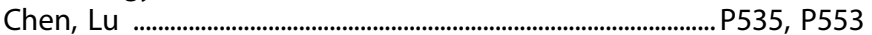

Chen, Peijun ................................................................................................... P17

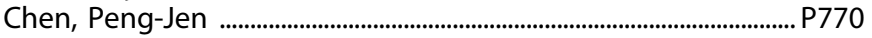

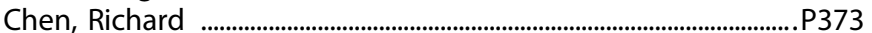

Chen, Shuo .............................................................................. P569, P814

Chen, Siwei ................................................................................................

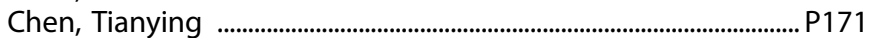

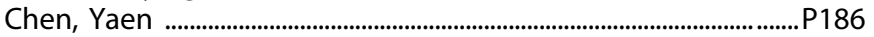

Chen, Yen-Chu ...................................................................................P623

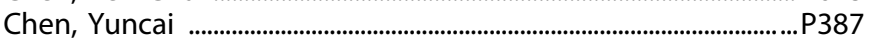

Cheng, Eileen ........................................................................................P535

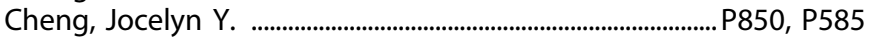

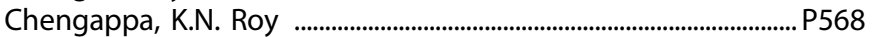

Cherif, Aboubacar .............................................................................................. P198

Chesler, Elissa J ....................................................................................... P199

Cheung, Victoria ......................................................................................... P10

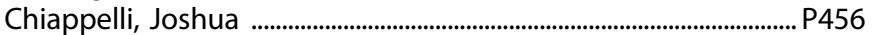

Chidomere, Chinenye ............................................................................... P745

Childress, Anna Rose . . . . . . . . . . . . . . . 7

Chin, Eunice ...……………………………………………………...P396

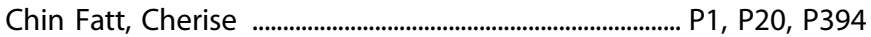

Chin-Fatt, Cherise .................................................................... P241

Chioma, Vivian ........................................................................................... P6 628
Chisholm, Alexandra ............................................................................ P742

Chitre, Apurva ........................................................................................ P705

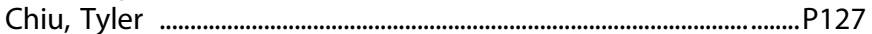

Cho, Sunghye ...............................................................................................P496

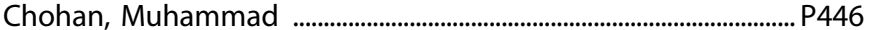

Choi, Doo-Sup .......................................................................... P662, P754

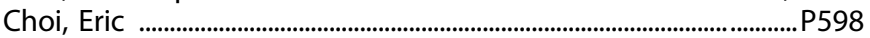

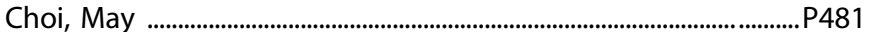

Choi, Sujean ............................................................................................P813

Cholewa, John .......................................................................................... P506

Choo, Tse Hwei ................................................................................................ P525

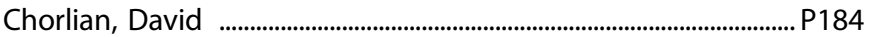

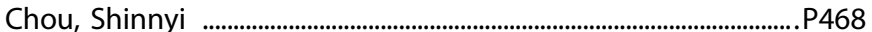

Christensen, Brooke ................................................................................. P629

Christian, Robert J. ....................................................................................... P768

Christina, Barr .....................................................................................................P691

Christine, Lee ..............................................................................................P334

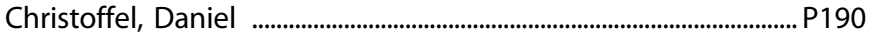

Christopher, Laughlin .................................................................................... P695

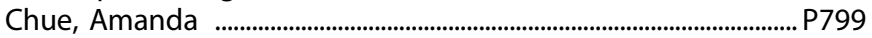

Chun, Thomas …................................................................................. P639

Cihakova, Daniela .................................................................................... P569

Cirilo, Anthony ……………………….....................................................P29

Cisneros, Irma ...........................................................................................P4

Cissé, Yasmine-marie .............................................................................28.2

Clancy, Julie ................................................................................... P280, P286

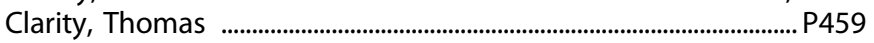

Clark, Kelly .......................................................................................................P495

Clark, Nicholas ............................................................................................... P596

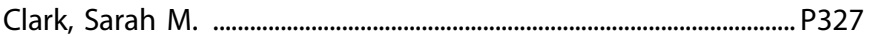

Clark, Uraina .........................................................................................P430

Clarke, Aaron ...............................................................................................P434

Clarke, Toni-Kim ............................................................................... P778

Clauss, Nikki ..................................................................... P69, P671

Clayton, Anita ..................................................................P222, P223, P224

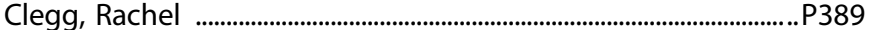

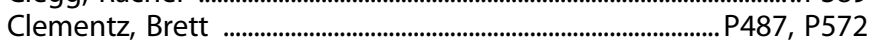

Clinical Consortium, INTRuST ................................................................. P88

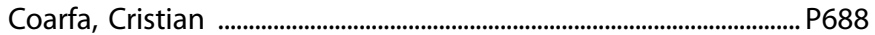

Coates, Kennedy ....................................................................................... P294

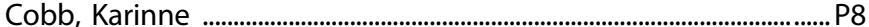

Cochran, David ......................................................................................... P163

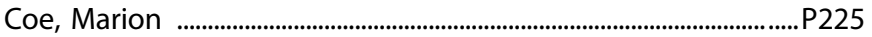

Coelho Milani, Ana Carolina ....................................................................P149

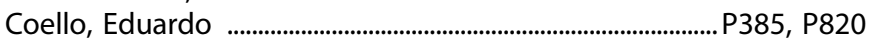

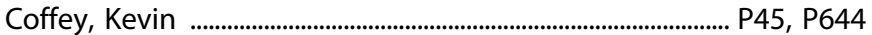

Coffman, Brian ………………………………………………………….... P5 51

Cogan, Elizabeth ....................................................................................... P371

Cohen, Justine ..................................................................................... P326

Cohen, Talia .....................................................................................................P505

Cohen, Trevor ..........................................................................................P465

Cohen, Zsofia ...........................................................................................P145

Cohen-Gilbert, Julia ...................................................................................P169

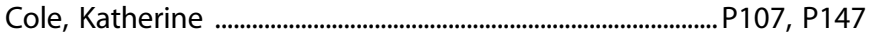

Cole, Rebecca H. ............................................................................................... P735

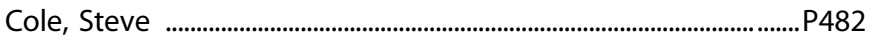

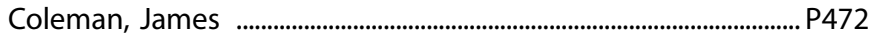

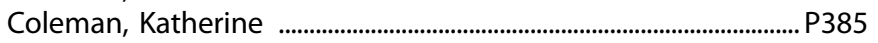

Coleman, Michael ................................................................. P565

Coley, Austin ...............................................................................................P267

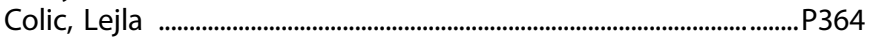

Collado-Torres, Leonardo ........................................................................P276

Collens, Sarah I. ......................................................................................... P4 41

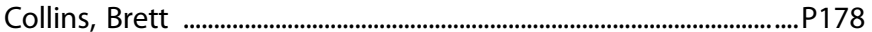

Collins, Gregory ...................................................................................................P776

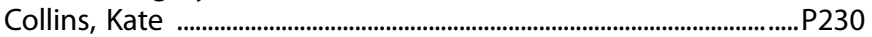

Collins, Katherine ............................................................... P68, P280, P286

Collins, Kelsey ................................................................................................P436 
Colucci, Laura

P453

Conelea, Christine

P150, P421

Cong, Yan

P496

Congdon, Eliza

P695

Conlisk, Dana

P677, P701

Conn, P. Jeffrey

P599, P655

Consortium, GEMRIC ...........................................................................P810

Consortium, International Brain pH Project ....................................P508

Constable, Todd .....................................................................................P291

Constantino, Nicholas .....................................................................P621

Contreras, Katherine ....................................................................................... P627

Cooke, Megan ........................................................................................ P606

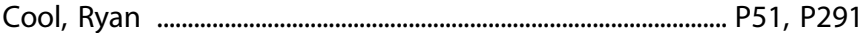

Cooley, Cameryn .................................................................................. P210

Coombs, Brandon J ...............................................................................P302

Cooper, Alexis .....................................................................................................P794

Cooper, Ashley ………………………………………..........................P169

Cooper, Jessica ...................................................................................... P310

Coppola, Andrea ............................................................................................ P36

Corcoran, Cheryl ......................................................................................... P98

Corder, Gregory ......................................................................................P339

Cordon-Cardo, Carlos ............................................................................P178

Corkrum, Michelle ............................................................................... P597

Cornblatt, Barbara A ................................................................................ P545

Corona, Winston ......................................................................................P123

Correa-Fernandez, Virmarie ................................................................P790

Correll, Christoph ...................................................32, P288, P373, P493

Cosgrove, Kelly ………………………........P58, P592, P668, P766, P783

Cosmo, Camila ................................................................................................ P384

Cossio, María José ................................................................................... P343

Costa, Ana Paula ........................................................................ P255, P275

Costa, Elson Alves ................................................................................... P407

Costa, Karla C.M. .................................................................................... P424

Costa, Renzo .....................................................................................................P297

Costa, Vincent ............................................................................................... P794

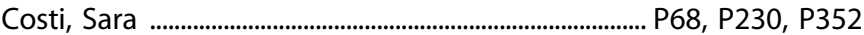

Côté, Sylvana ...................................................................................................P153

Cotel, Marie-Caroline .................................................................................P463

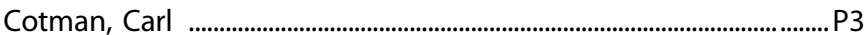

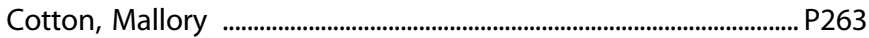

Cottone, Pietro .............................................................................................. P649

Courtet, Philippe ....................................................................................... P296

Covarrubias, Obdulia ......................................................................... P412

Covault, Jonathan ......................................................................................... P630

Covington III, Herbert .............................................................................. P635

Cowan, Christopher ...................................... 29, 29.1, P618, P712, P713

Cox, Sylvia ......................................................................................................P153

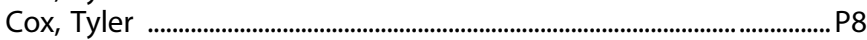

Craddock, Cameron ..............................................................................P793

Creed, Meaghan ......................................................................................... P182

Creutzberg, Kerstin Camile .....................................................P134, P320

Crippa, Jose A. .......................................................................................... P424

Crist, Richard ................................................................................ P339, P753

Croarkin, Paul .......................................................... 9, P144, P349, P418

Crook, Caitlin ...............................................................................................P677

Crow, Andrew .......................................................................................P495

Crow, Tim ..................................................................................................P487

Crowley, James .............................................................................................. P445

Crowley, Nicole ........................................................................................... P666

Crowley, T. Blaine .......................................................................................... P495

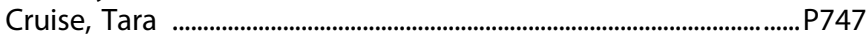

Cruz-Martin, Alberto .......................................................................................P331

Cruz-Sanchez, Arely ..................................................................................... P166

Cuarenta, Amelia .........................................................................................P737

Cubells, Joseph ................................................................................................. P1 12

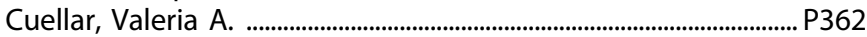

Cuellar-Barboza, Alfredo ............................................................................ P302

Cui, Xiaohong .................................................................................................P723
Cui, Zaixu

P148, P214

Cullen, Kathryn ....................................................................................... P817

Cullum, C. Munro ..................................................................................... P406

Culver, Paul

... 667

Cunningham, Kathryn

P412, P563, P636, P761

Cushnie, Adriana ....................................................................................P277

Cusimano, Michael ..................................................................................... P425

Custódio, Carlos Henrique Xavier ......................................................P407

Cutler, Andrew ............................................................................................ P271

Cvetkovska, Vedrana ................................................................................. P295

Czarapata, Jasmin .......................................................................................... P478

Czesak, Francesca .................................................................................. P626

D'Ambrosio, Shannon ............................................................................ P681

D'Angelo, Eugene ................................................................................. P512

D'Ascenzo, Marcello .................................................................................... P308

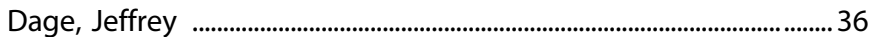

Dagher, Alain ............................................................................................P153

Dai, Feiyang .............................................................................................P404

Dai, Yulin .....................................................................................................P35

Dallanoce, Clelia ....................................................................................... P642

Daly, Rebecca ..............................................................................................P482

Damaj, Imad ..............................................................................................P627

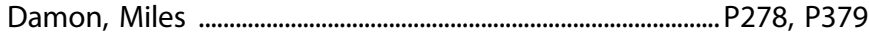

Dan, Shu .............................................................................................. P96

Daniel, David .................................................................................... P160, P554

Daniels, Emily ............................................................................................P569

Daniels, Teresa ............................................................................................P44

Daniels (Ret), COL Colin ......................................................................... P777

Dardenne, Laurent .......................................................................................... P424

Darrow, David ................................................................................................P324

Darwish, Mona …………………………………………….........P490, P518

Das, Priyanka ............................................................................................P195

Daskalakis, Nikolaos ...........................................................P91, P93, P290

Daskalakis, Zafiris ..................................................P314, P392, P418, P497

Datta, Abhishek ..................................................................................... P374

Datusalia, Ashok K. ..................................................................................... P87

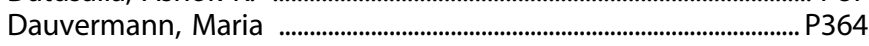

Dauvilliers, Yves ...................................................................................... P582

Davatzikos, Christos .................................................................................... P571

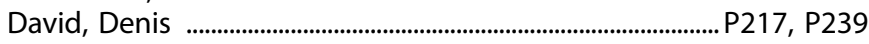

Davidson, Michael ....................................................................... 19, P546

Davignon, Lisa-Marie .................................................................................. P81

Davini, Weston ........................................................................................... P617

Davis, Catherine ..........................................................................................P651

Davis, Danielle ............................................................................................. P790

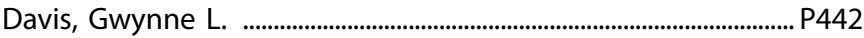

Davis, John ......................................................................................................P75

Davis, Lea K. ...........................................................................................P746

Davis, Margaret ............................................................................ P51, P291

Davis, Michael ................................................................................................... 36

Davis, Robert ....................................................................P209, P319, P373

Davis-Reyes, Brionna .................................................................................. P804

Daws, Lynette ............................................................................... P69, P671

Day, Jeremy …………………………..................................29, 29.2, P689

Dayan, Peter ............................................................................................P441

De Abreu E Gouvea, Andrea ................................................................P147

de Angelis, Paolo ....................................................................................... P254

De Aquino, Joao .................................................................................. P660

De Baun, Heloise .....................................................................P525, P574

De Biasi, Mariella ............................................................................................. P686

de Dios, Marcel ............................................................................................ P790

de Guglielmo, Giordano ................................................P677, P701, P759

de Guzman, Samantha ..................................................................................P607

de la Fuente Revenga, Mario ...............................................................P348

de la Monte, Suzanne ................................................................................. P44

de Laat, Bart ...............................................................................................................P766

de Lacy, Nina ................................................................................................ 3.1

de Lecea, Luis ......................................................................................................P729 
de Leon, Mony

De Luca, Vincenzo

P409

De Martin, Sara ...

P479, P579

de Moura, Fernando

P315

De Oliveira Sergio, Thatiane

P760

P604

De Peralta, Sharon

de Quevedo, Joao

De Rosa, Federico

de Schipper, Elles

de Wit, Harriet

De Witte, Lotje

Deakin, Bill

Dean, Andy

P83

P255, P275

P320

P445

P682, P695, P818

P178

.52 .4

P695

.P512

P371

DeBattista, Charles

P51

Debski, Jessica ............................................................................................. P607

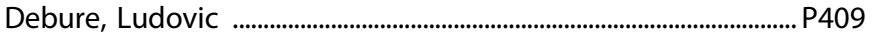

Dedic, Nina ..................................................................................................P290

Deems, Nicholas

DeGiosio, Rebecca

P16

P536

DeKarske, Daryl .

P490, P518

Delano-Wood, Lisa

DelBello, Melissa

Delgado, Julian

P25

DellaValle, Brian

Dell'Osso, Bernardo

Delmar, Dylan

Deloménie, Claudine

Del'Orco, Michela

DeLorenzo, Christine

DeLuca, Emily

DeMartinis, Nicholas

Demiral, Sukru

Demuth, Mara

den Ouden, Hanneke

Deneen, Benjamin

Deng, Zhi-De

Dennis, Evan

Dennison, Jessica

Denny, Christine

Denny, Christine Ann

Deptula, Andrew

Der-Avakian, Andre

DeRosse, Pamela

Desarkar, Pushpal

Deshpande, Rangaprakash

Desland, Fiona

Desta, Zeruesenay

DeTaboada, Luis

Detera-Wadleigh, Sevilla

Detre, John

Deutsch, Zoey

Devanand, Davangere .

Devarakonda, Kavya

DeVille, Danielle

Devillers, Olivia

DeVito, Elise

Devlin, Benjamin

Devlin, Bernie

DeVries, Brittany

Dhar, Aamina ...

Dhaynaut, Maeva

Dhindsa, Ryan .

Di Bonaventura, Maria

di Giannantonio, Massimo

Di Marco, Tobias

Di Martino, Adriana

P418

P352

P159

..P280, P286

P123

P148
Dias, Elisa

P525

Diaz, Joel

P187

Diazgranados, Nancy

Dichter, Gabriel

Dick, Danielle

Dickerson, Faith

Dickerson, Kathryn

Dickie, Erin

Dickinson, Abigail

Dickinson, Dwight

Didier, Paige

Diehl, Maria

Dienel, Samuel

Diener, Edward

Dietz, David

DiFeliceantonio, Alexandra

Dighe, Niharika

Diler, Rasim

Dillon, Daniel

Ding, Wanqiao

Ding, Ying

Ding, Yu-Shin

Diniz, Breno

Diniz, Juliana

Dixon, H. Drew

Dixon, Hayley

Dmochowski, Jacek P.

do Couto Bordignon, Pedro

Do Monte, Fabricio H.M.

Dobbin, Josh

Dobrowolny, Henrik

Docherty, Anna

Dodd, Emily

Doherty, James

Dohi, Eisuke .

Dokas, Thomas

Dolan, Siobhan

Dolder, Patrick

Dols, Annemiek

Dolzani, Samuel

Domi, Ana

P285, P678, P680, P687, P692, P700

P388, P789

P184, P703

Domi, Esi

Dominick, Kelli

Domino, Edward

Donahue, Greg

Donaldson, Zoe

Donegan, Jennifer

Donello, John

Dong, Cheng

Dong, Erbo

Dong, Pengfei

.... 33

P122

P418, P497

P187

P450, P539

P571

P33

P484

P281

P612

P194

P663

P282, P363

$P 251, P 357, P 388$

P613

P549

... P19

P9, P248, P344, P354

P444

P53, P72

...P286

P295

P308, P745

..P244

P577

P819

P265

P222, P223, P224, P271

..P830

P336

P178

P824

P17

P266

...P36

P36, P711

P173, P428

P102

P664

Dong, Tri

Dong, Yan ........................................................................................30, P613

Donovan, Emily ........................................................................................ P583

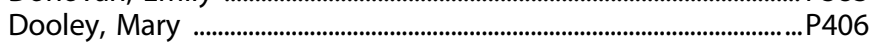

Dorofeikova, Maria ..................................................................................... P89

Doruk Camsari, Deniz ............................................................................ 9.3, P144

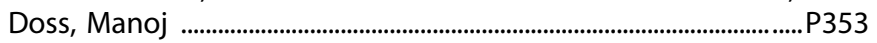

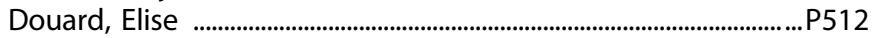

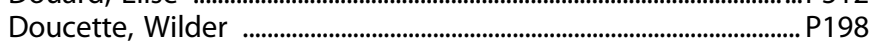

Dougherty, Joseph ...............................................................................P220

Dracheva, Stella _.................................................................................................. P461

Drake, Robert ...................................................................................................P773

Dremencov, Eliyahu ...............................................................................P205

Drevets, Wayne

Drossel, Gunner

P288, P330, P377

.

Drysdale, Andrew .............................................................................................. P28 
D'Souza, Deepak

Du, Xian

Duarte, Cristiane

...P514

Dubey, Harikesh

49, P149

P795

P421

Dubroff, Jacob ........................................................................................... P819

Dufour, Brett ............................................................................................P644

Dugan, Elizabeth ......................................................................................... P420

Dugovic, Christine ..................................................................................... P617

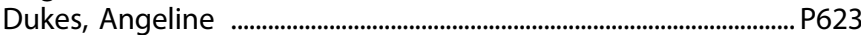

Dulcey Garcia, Andres ............................................................................. P547

Dunayevich, Eduardo ...............................................................P514, P560

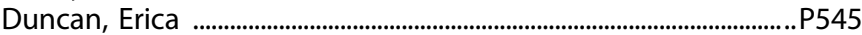

Duncan, Wallace ........................................................................................ P402

Dunlop, Boadie ...........................................................361, P369, P371

Dunn, Erin .....................................................................................................P151

Dunn, Kelly ..............................................................................................P667

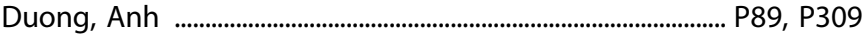

Duong, Duc ................................................................................................... P93

Duperrouzel, Jacqueline ..................................................................... P587

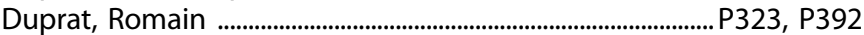

Durand-de Cuttoli, Romain ................... P177, P301, P342, P352, P657

Durgam, Suresh ......................................................................P319, P373

Duval, Elizabeth ............................................................................P748, P801

Duvvuri, Sridhar .......................................................................... P493, P500

Dvorak, Nolan M. ......................................................................... P308

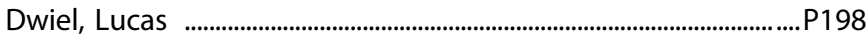

Dwomoh, Dorothea ..........................................................................P430

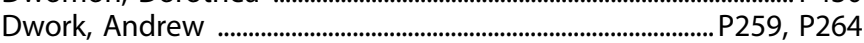

Dworkin, Jordan ............................................................................................ P149

Dwyer, Heather ............................................................................................ P440

Dyer, Samya ............................................................................... P23, P170

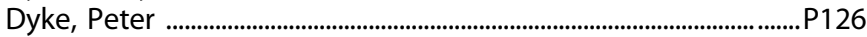

Dzemidzic, Mario ..........................................................................................P707

Dzierzewski, Joseph ................................................................................... P583

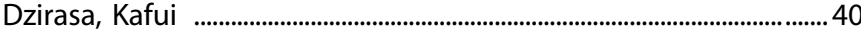

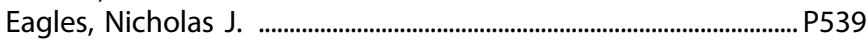

Eaton, William ........................................................................................P569

Ebdrup, Bjørn ...............................................................................P526, P542

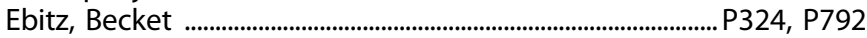

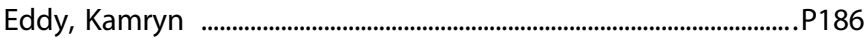

Edenberg, Howard J. .................................................................................. P184

Edgar, Chris J ..........................................................................................P517

Edlow, Andrea ........................................................................................... P151

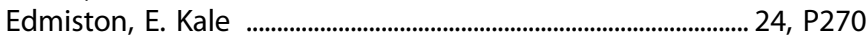

Edwards, John ......................................................................................P319

Egazarian, Alexis ........................................................................................... P7 739

Egervari, Gabor ......................................................................................... P664

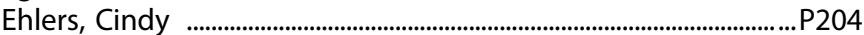

Ehrensaft, Diane .............................................................................................. 24

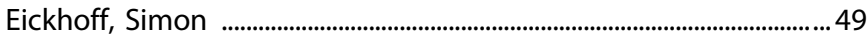

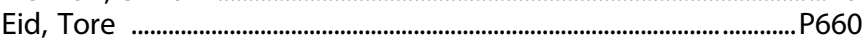

Einhorn, Nathan ................................................................................ P269

Eisenberg, Daniel ...........................................................147, P450, P478

Eisenlohr-Moul, Tory ..................................................................... 53.1, P789

Ekhtiari, Hamed ................................................................................ 7, P652

El Fakhri, Georges ..................................................................................... P500

Elaine, Wang .............................................................................................P334

Elam, Hannah ......................................................................................P489

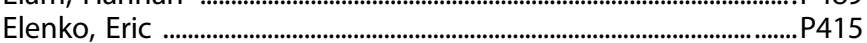

Elhasid, Tal ...................................................................................................P544

Elhassa, Salma _................................................................................................ P342

Elizabeth, Hohmann .................................................................................... P3 P34

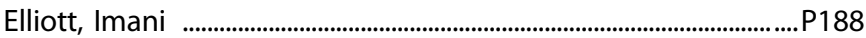

Elliott, Mark ..........................................................................................................P571

Elson, Sarah L ......................................................................................P746

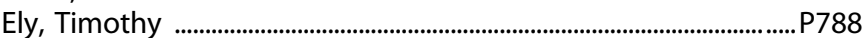

Elyaderani, Amir ................................................................................... P634
Emili, Andrew

.P694

Emily, Hartwell

P753

Engelke, Douglas

P308, P745

Engeln, Michel ........................................................................................ P598

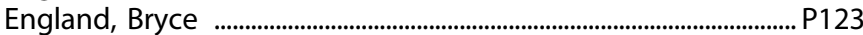

Engleman, Eric ……………………………………………………………... P7

Enriquez, Kristen .......................................................................................P171

Enten, Lauren ..........................................................................................P793

Entringer, Sonja ......................................................................................P106

Eom, Kelly .................................................................................................P122

Epel, Elissa ..............................................................................................P2 11

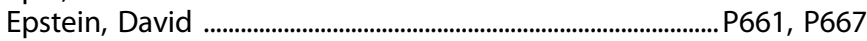

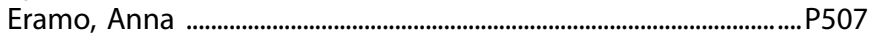

Erbes, Christopher ............................................................................... P247

Ereshefsky, Sabrina ........................................................................... P555

Erhard, Emily .............................................................................................P649

Erias, Kyra .................................................................................................P612

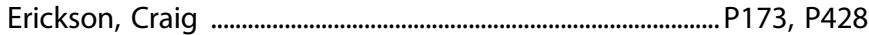

Erickson, Greg ................................................................................................. P3

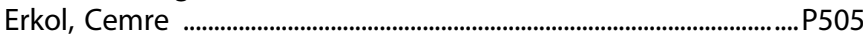

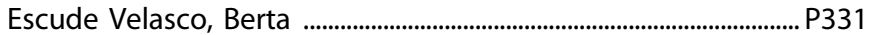

Eshel, Neir ................................................................................................ 24, 47

Esterlis, Irina .................................................................... P51, P291

Estill, Molly ..............................................................................................P708

Ettaro, Robert ..........................................................................................P338

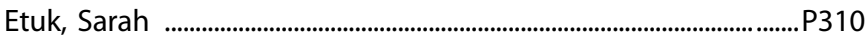

Evans, Christopher .................................................................................. P608

Evans, Kathryn ...........................................................................................P778

Evans, Rebecca ................................................................................................. P560

Evanski, Julia .............................................................................................P111

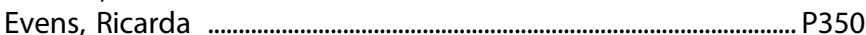

Evers, Audrey ................................................................................ P68, P230

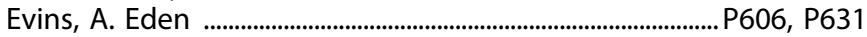

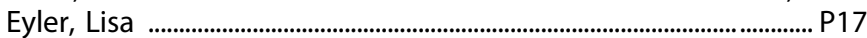

Fabro, Alexandre T. .................................................................................. P424

Facchinetti, Roberta ....................................................................... P87

Fadok, Jonathan ………………………………………................... P89, P309

Faerman, Afik ...........................................................................................P176

Fair, Damien ............................................................................. 40, 49, P106

Fajemiroye, James .................................................................................... P407

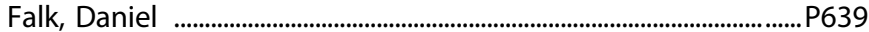

Falke, Conner .........................................................................................P817

Fall, Dylan ...............................................................................................P328

Fallon, Isabella ........................................................................................... P2 266

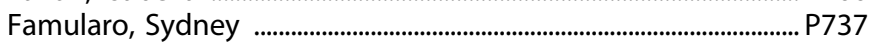

Fan, Rong ...............................................................................................P264

Fang, Jidong .........................................................................................P613

Fang, $\mathrm{Yu}$....................................................................................................

Fani, Negar ................................................................................ 27.1, P64

Fannon, McKenzie ...................................................................................... P677

Fanous, Ayman ..................................................................2226, P477, P575

Fant, Reginald ......................................................................................... P315

Faraco, Carlos ...........................................................................................P429

Faraj, Mohammed ............................................................................................ P111

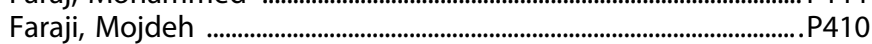

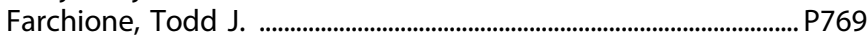

Fareed, Jawed ..................................................................................... P297

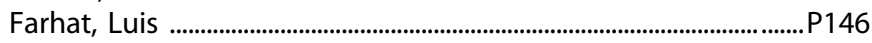

Farinelli, Federica ....................................................................................... P539

Farokhnia, Mehdi ............................................................................................... P726

Farrelly, Lorna .........................................................................................P417

Farris, Karen ..............................................................................................P33

Fatih, Parmis ............................................................................................... 144

Fatourou, Evangelia .................................................................................... P297

Faulkner, Monica ............................................................................................... P648

Faull, Peter ..............................................................................................P591

Fava, Maurizio ............................................................................. P361

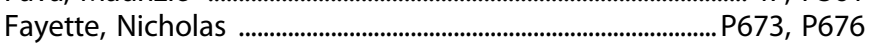


Fazlali, Zeinab

Feczko, Eric

Fede, Samantha

Feder, Adriana

Federoff, Monica

Fedota, John

Feilding, Amanda

Feinn, Richard

Felder, Christian

Feldman, Dana

Felger, Jennifer

Felix-Ortiz, Ada

Feliz, Samantha

Felsing, Daniel

Fenselau, Henning

Fenster, Robert

Feola, Brandee

Ferguson, Bradley

Fernandes, Brisa

Fernandez, Thomas

Ferranti, Anthony

Ferrarelli, Fabio

Ferrario, Carrie

Ferreira, Pedro Eugenio Mazzucchi Santana ......................................P675

Ferreira, Rafael Rinaldi .................................................................................. P424

Ferreira, Vania ................................................................................................P635

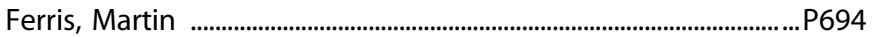

Fetterly, Tracy

Feusner, Jamie

Fields, Andrea

$14.1, \mathrm{P} 187, \mathrm{P} 439, \mathrm{P} 440$

(1)...P589

Fields, Sherecce ........................................................................................ P790

Fifer, William P.

Findling, Robert

Fineberg, Naomi

Finnemann, Silvia

Fischer, Corinne

Fischer, Kathryn

Fish, Kenneth

Fishel, Vanessa

Fitzgerald, Kate

Fitzgerald, Megan

Fitzgerald, Robert

Fitzpatrick, Sarah

Fiumara, James

Fix, Spencer

Flagel, Shelly

Flais, Ivana

Flanigan, Meghan

Flatow, Elie

Fleck, David

Fleischel, Erik

Fleischhacker, Wolfgang

P149

$P 160, P 200, P 202, P 238$

P434, P440

P80

P314

P510

P468, P484

P531

... 9.2

P472

P755

P171

P496

P669

P705

P400

42.1, P177, P645

P461

P397, P398

Flesher, Melissa .................................................................................. P8 815

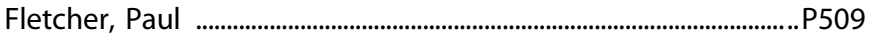

Flint, Alastair .........................................................................................................P314

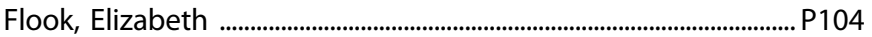

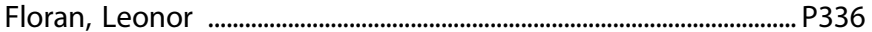

Flores, Cecilia ................................................................................................. 25

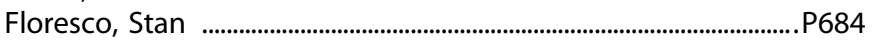

Flowers II, James _..........................................................................P294, P684

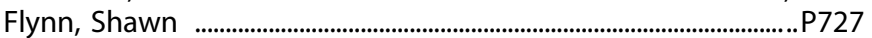

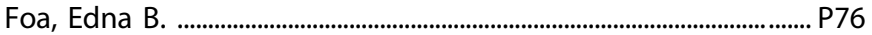

Foerde, Karin .................................................................................................. 196

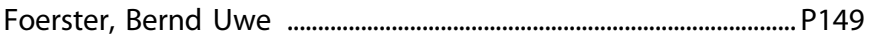

Fogelman, Nia ............................................................................... P593, P805

Foilb, Allison

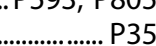

Foley, Ann ...............................................................................................P163

Folli, Franco ......................................................................................P315

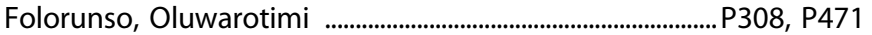

Foneska, Monica ...................................................................................... P385

Fonkoue, Ida ....................................................................... P53, P72

Fontanillas, Pierre .................................................................................... P746

Fonzo, Gregory ..............................................................................................P793

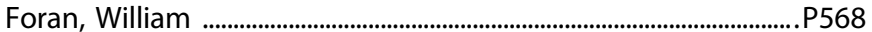

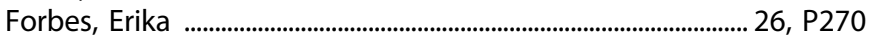

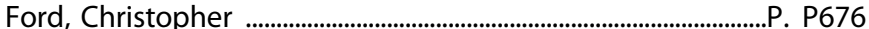

Ford, Judith ………………………………………........P470, P503, P561

Forester, Brent ............................................................................... P17, P371

Forlenza, Orestes ............................................................................. P17

Forman, Mark .............................................................................................P490

Foroud, Tatiana ................................................................................. P184

Forsyth, Jennifer ................................................................................................ P499

Forthman, Katherine ........................................................................... P242

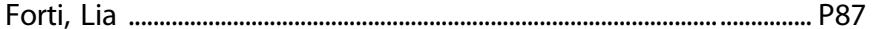

Fortin, Justine .................................................................................................... P81

Foster, Jane ....................................................................................................... P20

Foster, Simmie .............................................................................................. 4.4

Fournier, Jay ..................................................................................P243, P270

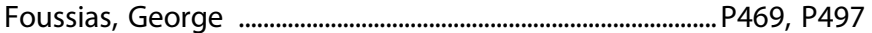

Fowler, Christie ...............................................................................P623, P642

Fowler, James ...............................................................................................P623

Fox, Andrew ................................................................................................ P78

Fox, Megan ..................................................................................................P598

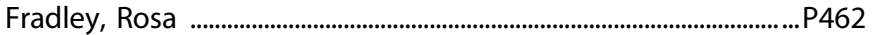

Fragale, Jennifer ...........................................................................................2. 20.2

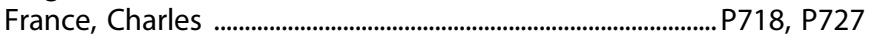

Francis, Sunday ................................................................................................ P150

Francis, Tanner ................................................................................................. P52

Francisco, Ana Paula .......................................................................... P440

Francisco, Taylor .................................................................................... P84

Franco, Alexandre Rosa .........................................................................P675

Franco, Daniela .................................................................... P598, P704

Frangou, Sophia ..................................................................... P22, P162

Frank, Guido .............................................................................. 14, 14.4

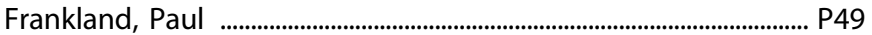

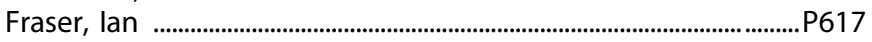

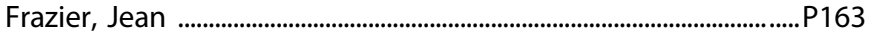

Frederiks, Kevin ................................................................................... P31

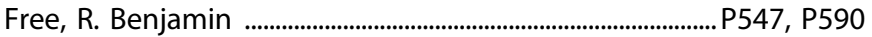

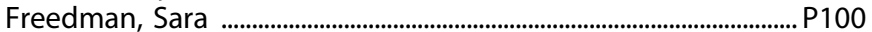

Freimer, Nelson ....................................................................................... P695

French, Delores A. .................................................................................. P78

Frere, Justin ..................................................................................................... 419

Freyberg, Zachary ..........................................................P360, P427, P765

Friederici, Angela D ......................................................................................P141

Friedman, Allyson ..................................................................................... P177

Friedman, Naomi .................................................................................. P341

Friend, Samantha .................................................................................. P38

Fries, Gabriel R. ............................................................P275, P362, P688

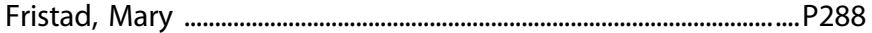

Fritch, Emma ................................................................................................ P35

Fritsch, Emma …………………………………….............. P278, P379

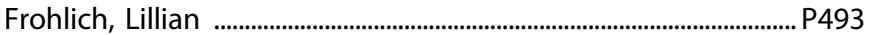

Fromme, Kim ............................................................................................P332

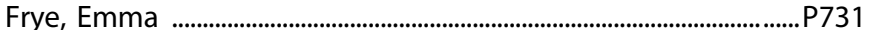

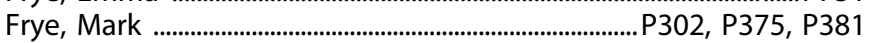

Fryer, Susanna ...................................................................................... P561

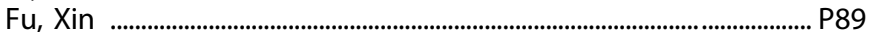

Fuccillo, Marc .............................................................................................P787

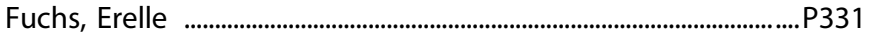

Fuchs, Rita A. .........................................................................................P768

Fuchs, Sarah ...........................................................................................P533

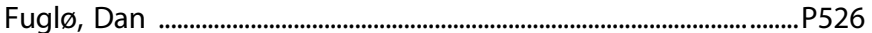

Fujita, Masayo ..............................................................................................P426 
Fujita, Mika .........................................................................................................P102

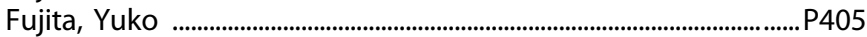

Fulenwider, Hannah ........................................................................... P263

Fullard, John .................................................................................................P461

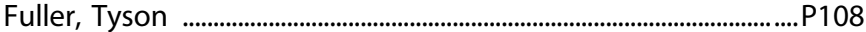

Fultz, Allison ..........................................................................................P809

Fundin, Bengt ........................................................................................P445

Furqan, Muhammad ........................................................................... P252

Fusi, Stefano .................................................................................................P537

Futamura, Rita .......................................................................................... P657

Futamura, Takashi ....................................................................................... P2 208

Gabbay, Vilma ..................................................................................... P674

Gaddis, Andrew ........................................................................................ P784

Gage, Fred ...............................................................................................P401

Gagnon, Leona ......................................................................................P199

Gai, Guan-chen ........................................................................................P522

Gainham, George ................................................................................P156

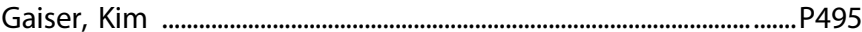

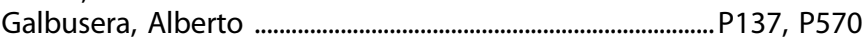

Galderisi, Silvana ...................................................................................... P560

Gale, Jennifer ............................................................................................P472

Gallagher, R. Sean ................................................................................ P495

Gallego, Juan .....................................................................................P455

Gallo, Eduardo .............................................................................................. P2 207

Gameroff, Marc .....................................................................................P299

Gandhi, Pauravi ........................................................................................... P681

Gandhi, Rushali .................................................................................... P10

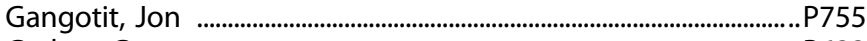

Garber, Gregory ................................................................................................. P633

Garcia, Benjamin ....................................................294, P417, P664, P708

Garcia, Christian ............................................................................................... P610

Garcia, Eliana Korina ............................................................................ P128

Garcia, Ronald ................................................................................... P326

García, Benjamin ...................................................................................... P221

Garcia-Keller, Constanza .............................................................. P67, P717

Garcia-Rada, Maria Fe ........................................................................... P272

Garcia-Reyes, Ruben ............................................................................. P39

Garcia-Romeu, Albert ...............................................................................P790

Gardea Resendez, Manuel ........................................................................ P302

Garden, Gwenn ..................................................................................P644

Gardier, Alain .............................................................................P217, P239

Garman, Heather .......................................................................................... P555

Garon, Juliet .............................................................................................P301

Garrett, Laren .................................................................................................P321

Garvey, Emily .........................................................................................P512

Garza, Maria ............................................................................................. P41

Gaspari, Sevasti .................................................................................P417, P730

Gates, Linda .............................................................................................P336

Gatta, Eleonora .................................................................................. P269

Gaudreault, Pierre-Olivier ...................................................................P752

Gaulden, Andrew ................................................................................ P722

Gauthier, Serge ................................................................................................... P10

Gautier, Karissa ......................................................................................... P113

Gaziano, J Michael .................................................................................P477

Ge, Shaoyu ........................................................................................P535

Ge, Tian .......................................................................................................P798

Ge, Tintting .........................................................................................P560

Gehring, William ............................................................................................ P4 436

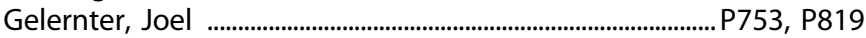

Gelfond, Jonathan ......................................................................................... P12

Genetti, Casie .........................................................................................P512

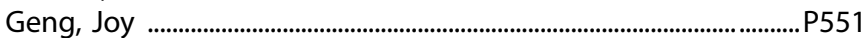

Genomics Consortium, Latin American ................................................P736

Geoly, Andrew .........................................................................................P176

George, Brianna ............................................................................................ P650

George, David ............................................................................................. P687

George, Grace .........................................................................................P167

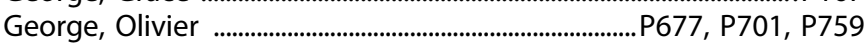

George, Susan P653

George, Tony ..........................................................55, 55.4, P179, P502

Georgiou, Polymnia .............................................................................. P327

Geramita, Matt ......................................................................................... P4

Gerlach, Andrew ........................................................................... P11, P74

Germain, Anne ......................................................................................... P825

German, Ramaris .......................................................................................... P37

Germine, Laura ............................................................................................ P78

Gerretsen, Philip ....................................................................................... P579

Gershon, Elliot .......................................................................38, 38.2, P572

Gersten, Maia B. ............................................................................P280, P286

Gertsman, Ilya ........................................................................................P755

Geske, Jennifer ............................................................................................ P762

Geurts, Aron ...........................................................................................P813

Geurts, Jeroen ....................................................................................... P218

Ghoshal, Ayan .............................................................................................. P472

Ghoshal, Soham .................................................................................... P601

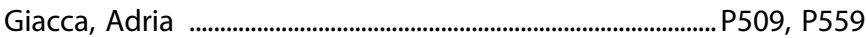

Giannotti, Giuseppe ..................................................................P602, P676

Giddens, Natasha .................................................................................... P692

Gigase, Frederieke ...................................................................................... P178

Gilbert, Kirsten ........................................................................................ P135

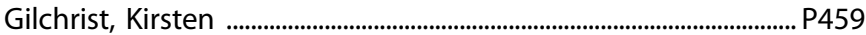

Gilchrist, Michelle A. .............................................................................. P149

Gildengers, Ariel ......................................................................................... P17

Gilder, David .................................................................................................P204

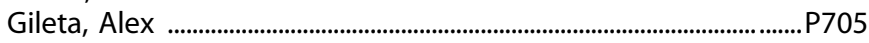

Gill, Mary Kay ............................................................................................P304

Gilles, Maria ...............................................................................................P350

Gillespie, Charles ........................................................P48, P53, P64, P72

Gilman, Jodi ..................................................................................P606, P631

Gilmartin, Marieke ........................................................................................ P8 813

Gilmer, Todd ..........................................................................................P555

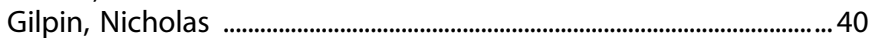

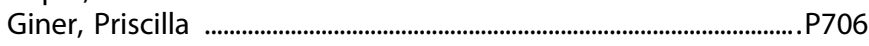

Gingrich, Jay ............................................................................. P149, P292

Gipson, Cassandra .................................................................................... P781

Girdhar, Kiran ..............................................................................................P461

Girelli, Tommaso …………………………………………………………..... P392

Girgenti, Matthew ......................................................................... P91, P94

Gitlin, David ...................................................................................................P326

Giusepponi, Maria Elena ......................................................................... P584

Giusti-Rodríguez, Paola ........................................................................P736

Gjedde, Albert ..............................................................................................P526

Glahn, David .........................................................................................P512

Glatfelter, Grant ............................................................................................. P732

Glauser, Liliane ............................................................................................ P75

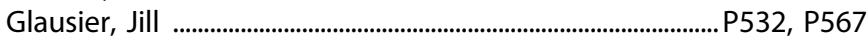

Gleason, Kelly .............................................................................................P380

Gleason, Theresa ......................................................................................... P4 P47

Gleissl, Teresa ................................................................................................P154

Glenthoj, Birte ....................................................................... 5524, P526, P542

Glicksberg, Benjamin ............................................................................ P62

Glimcher, Paul ................................................................................................. P58

Glover, Ebony ................................................................................................. 58

Glover, Elizabeth ................................................................................... P725

Gluck, Rachel .................................................................................... P48, P72

Goate, Alison ..............................................................................................P184

Gobin, Christina _........................................................................................ P721

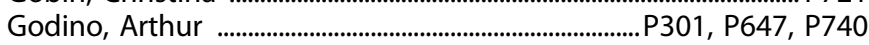

Godoy-Vitorino, Filipa ............................................................................... 33

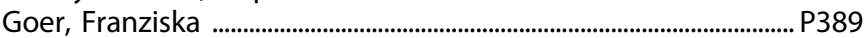

Goes, Fernando S. ......................................................................P276, P28

Goetschius, Leigh G. ........................................................................... P140

Goff, Allison .............................................................................................................. 3551

Goff, Donald ................................................................................................... 32

Gohel, Chiraag .................................................................................................. P138

Goke, Lt COL Kevin ......................................................................................... P77 
Gold, James

Goldberg, Samantha

Goldberg, Terry

Goldberger, Nehama

Goldfarb, Elizabeth

Goldman, Danielle

Goldman, David .

Goldman, Melissa

Goldman Bergmann, Carolina

Goldsmith, David

Goldstein, Benjamin

Goldstein, David

Goldstein, Jill

Goldstein, Rita

Goldstein, Stanley

Goldstein, Tina

Goldstein-Piekarski, Andrea N.

Goldwaser, Eric

Golovko, George

Gomes, Felipe

Gomez, Juan

Gomringer, Brian

Gong, Sheng

Gonzales, Ruben

Gonzalez, Jocelynn

Gonzalez, Raul

Gonzalez, Tiffany

Gonzalez-Heydrich, Joseph

Gonzalez-Maeso, Javier

Goodarzi, Hani

Goosens, Ki

Gordon, Joshua

Gordovez, Francis James

Gorelik, Aaron

Gorelik, Mark

Gorka, Stephanie

Gorodetsky, Charles

Gotts, Stephen

Gould, Georgianna G.

Gould, Todd

Gourley, Shannon

Gozenbach, Virgil

Gozzi, Alessandro

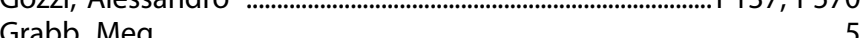

Grace, Anthony

Gracias, Alexa

Graeff, Johannes

Graff-Guerrero, Ariel

Graham, Alice

Graham, Danielle

Granata, Lauren

Granja, Karen

Grant, Caroline

Grant, Corena

Grant, Jazmine

Grant, Jon

Grant, Philip

Grantham, Emily

Grasser, Lana .

Grassi, Giacomo

Grassi-Oliveira, Rodrigo

Grattan, Rebecca

Grattan, Vince

Graur, Simona

Gray, John

Gray, Joshua C

Gray, Madelyn

Greden, John
P469, P551

... P748

48, P566

P544

P593

P364

P138, P351, P678, P680, P687, P700

P236

P440

P498, P545

P363

P566

4, P251, P326, P357

P625, P752

P694

P363

P386

P456, P530

P636

P268

P175, P233, P729

P108

P676

P681

P659

P587

P704

P512

15, P348, P627

P744

P68

$12,23,40, \mathrm{P} 128, \mathrm{P} 459$

P123

P393

...P393

P185, P260

P315

P799

P671

P233, P327

P622, P663

P495

P137, P570

P109, P268, P710

P413

P75

P453, P579

P106

... 36

P157

P587

P349

P180

P188

.. 1.3, P443

P253

P698

P130

P440

P134, P675

P555

P507

P270, P304

P471

P695

P785

.P371
Green, Charles P328

Green, Lisa

Green, Michael

P499, P515

Green, Tamar

P105

Greene, Natasha ........................................................................... P446

Greenstein, Dede ................................................................................................... P402

Greenwald, Julia ...................................................................................... P207

Greer, Tracy _..........P288

Gregg, Allison

Gregory, Michael

P406

Grice, Dorothy

P107, P147, P450, P478

Grieco, Matthew …………………………………………………………...... P75

Griffin, Claire ..... P605

Griffiths, Roland

Grinband, Jack

P353, P654, P784

Grinchii, Daniil

Grissom, Nicola

Grizzell, J. Alex

Grodin, Erica

Gross, Alexa L.

Gross, Robin

Group, EPI-CAL

P458

P205

58, P150, P324

P720

Grove, Tyler

Groves, Coltrane ................................................................................. P626

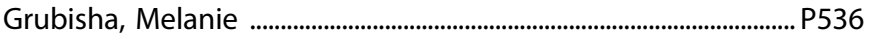

Grueter, Brad .......................................................................................................P65

Gründer, Gerhard ....................................................................................... P350

Gruskin, David ......................................................................................... P527

Grysko, Robert ............................................................................................... P3 336

Grzenda, Adrienne .......................................................................... P227

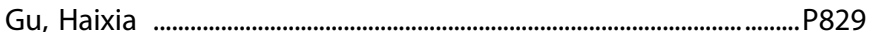

Gu, Pan ...........................................................................................................P4

Guarez, Barbara .....................................................................................P417

Guedes, Isabella ............................................................................................. P424

Gueguen, Maëlle .......................................................................................... P738

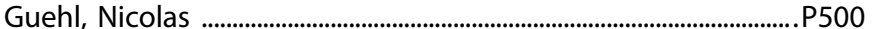

Gueorguieva, Ralitza ............................................................................ P660

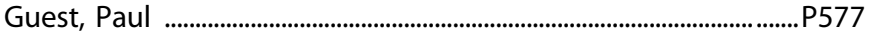

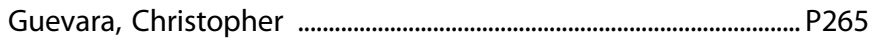

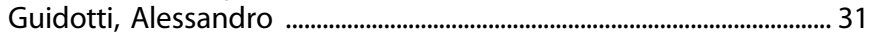

Guimaraes, Francisco S. ........................................................................P424

Gukasyan, Natalie ............................................................................................... 15

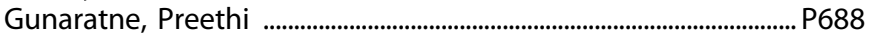

Gunawan, Tommy ...................................................................................P687

Gunaydin, Lisa ............................................................................ 50.2, P442

Gunn, Benjamin ...................................................................................... P114

Guo, Annie ............................................................................................................P415

Guo, Jessica .................................................................................................P496

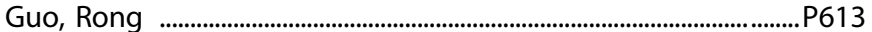

Guo, Zhenglin .......................................................................................P522

Gupta, Sonya .........................................................................................P791

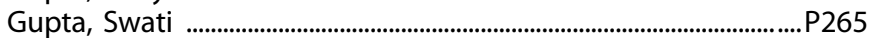

Gur, Raquel

5, P131, P148, P495, P571

Gur, Ruben

P76, P131, P148, P495, P571

Gur, Tamar

28.1

Gurung, Ashik ...............................................................................................P708

Guthman, Eartha .............................................................................................. 24

Gutierrez, Gilmar ....................................................................................... P382

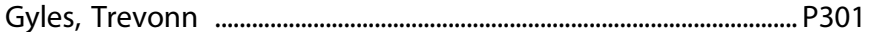

Haas, Shalaila ........................................................................................... P22

Haber, Suzanne ..........................................................35, 35.1, P201, P441

Habets, Philippe ......................................................................................... P318

Hack, Laura .....................................................................................................P253

Hafeman, Danella .......................................................................................... P363

Haft, Mark .......................................................................................................P449

Hagan, Kelsey ...........................................................................................P193

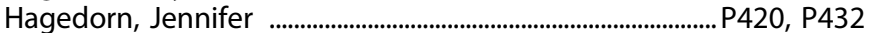

Hagenauer, Megan ....................................................................................... P336 
Hagerty, Sarah

P828

Haghighi, Fatemeh

Hagihara, Hideo

P508

Hagino, Yoko

P426

Hahn, Britta

P551

Hahn, Emma

P310

Hahn, Margaret

Hain, Daniel

Hajali, Danny

Hajeissa, Alaa

Hajjar, Emily

Hakimi, Shabnam

Haklai, Ziona

Hakusui, Christopher

Halaris, Angelos

Halko, Mark

Hall, Julia

Hall, Mei-Hua

Hall, Nancy

Hall, Victoria

Hallak, Jaime E.C.

Haller, Simone ..

Halvorsen, Aaron

Halvorsen, Matthew

Hamaguchi, Kosuke

Hamborg, Madeline

Hamidovic, Ajna

Hamilton, Holly K.

Hamilton, Peter

Hamm, Jordan

Hammack, Robert

Hammock, Bruce D.

Hammond, Taeah

Hammoud, Abdul

Han, Ming-Hu

Han, Sifei

Han, Xue

Hanania, Taleen

Hanlon, Colleen

Hanna, Gregory

Hänsel, Katrin

Hansen, Lars

Hanson, Elizabeth

Hanukaai, Shadi

Happe, Cassandra

Hardee, Jillian

Harden, K. Paige

Hardi, Felicia

Harms, Matthew

Harnett, Nathaniel

Haroon, Ebrahim

Harp, Nicholas

Harper, Jennifer

Harrell, Dameon

Harrewijn, Anita

Harris, Alexander

Harris, Robert Adron

Harrison, Dannielle

Hart, Kim

Hartley, Catherine

Hartmann, Jakob

Hartwell, Emily

Harvey, Brandon

Harvey, Philip

Harvin, Teavian

Harwell, Corey

Hasan, Khader

Hasantash, Maryam
P453, P509, P559

P371

... P41

P380

P633

P122

P544

P555

P297, P305

...P475

P251, P357

P474, P522

P585

P609

P424

P799

P609

P445

P823

P107, P147, P478

P335

P545

P301, P723

P464

... P69

P189

P436

P550

P177, P252

P415

...30.1

P432

55, 55.2

P436

P496

P542

P560

P608

P549

P748

P703

P140

P566

27.2, P788

P310

P826

.. P28

P324

P43

..P128, P216, P446, P537

P698

P310

P777

P54

P290

P819

P79

P477, P575

P615

...11.1

P803

P346
Hasegawa, Yuto P558

Hashimoto, Kenji

P405

Hastings, Lyndsay _P731

Haubrick, Kayla ............................................................................................. P206

Hauser, Sheketha ................................................................................... P774

Hausmann, Frances ..................................................................................... P331

Havener, Edmund ...................................................................................... P40

Hawco, Colin

P232, P418, P469, P497

Hawes, Samuel .......................................................................................... P587

Hayden, Ashley ................................................................................................ P15

Haynes, Sherod ............................................................................................ P252

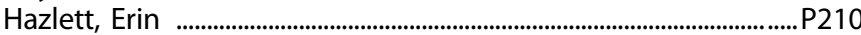

Hazlett, Mariah .................................................................................................... P609

$\mathrm{He}$, Hao ......................................................................................................P199

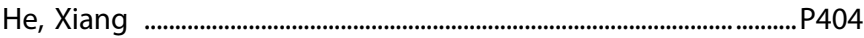

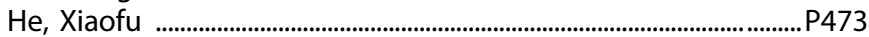

Heads, Angela ........................................................................................... P790

Heberle, Bernardo ........................................................................... P134

Heckers, Stephan .......................................................................................... P486

Hegde, Rachal .......................................................................................... P430

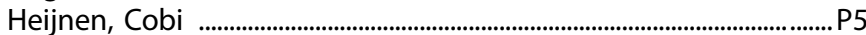

Heilbronner, Sarah .................................................................................. P277

Heilig, Markus .................................................46.1, P36, P696, P711, P771

Heinrich, Hadley ......................................................................................... P556

Heinsbroek, Jasper ........................................................P602, P673, P676

Heissel, Soeren .......................................................................... P744

Hejazi, Nadia .................................................................................................P402

Helin, Kristian .........................................................................................P221

Helion, Chelsea .............................................................................................. P71

Hellemann, Gerhard .................................................................... 5515, P695

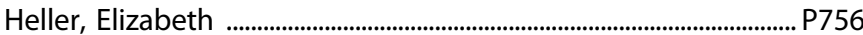

Hemby, Scott ...............................................................................................P590

Hempel, Casper ..................................................................................... P542

Hen, Rene ...............................................P50, P56, P128, P217, P244

Hendershot, Christian .............................................................................. P767

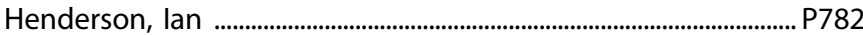

Hendler, Talma ........................................................................................... P447

Hendrickson, Rebecca .............................................................. P99, P777

Hendrickson, Tim ....................................................................... P511

Henningfield, Jack ...........................................................P225, P315, P790

Henriquez-Belmar, Francisca ................................................................. P343

Henry, Stefanie ............................................................................................ P195

Henry, Teague ............................................................................................ P282

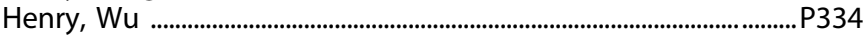

Herkenham, Miles ........................................................................................ P329

Herman, Alexander ..................................................................................... P324

Herman, James ....................................................................................... P300

Herringa, Ryan ............................................................................. P63, P167

Herrmann, Nathan ................................................................... P314

Hertzel, Sara .................................................................................................P728

Heslegrave, Amanda ............................................................................... P306

Hess, Evan .........................................................................................P813

Hesselbrock, Victor ....................................................................P184, P204

Hetherington, Hoby ............................................................................... P284

Hetrick, William ....................................................................................... P514

Heustis, Marilyn .................................................................................P631

Heyn, Sara ..........................................................................................P167

hibicke, Meghan ....................................................................................... P229

Hickey, Mary Beth ....................................................................................... P363

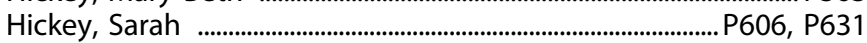

Hicks, Stephanie ...................................................................................... P276

Higginbotham, Jessica A. .....................................................................P768

Higley, J. Dee ..............................................................................................P691

Hikida, Takatoshi .................................................................................... P823

Hildebrand, Mariah ........................................................................................ P5 532

Hiler, Daniel J. ........................................................................................... P539

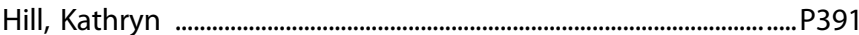

Hill, Matthew ................................................................................................ P49 
Hillemacher, Thomas

P533

Hillmer, Ansel

Hinds, Nicole

Hinds, Walter

Hines, Rachel ...

Hing, Benjamin

Hipolito, MariaMananita

Hirsch, Lorna

Hirst, Kathy

Hitora-Imamura, Natsuko

Hixon, Mark

Ho, Ada Man-Choi

Ho, Beng-Choon

Ho, Diana

Ho, Shaun

Hobbs, Taylor

Hodes, Georgia

Hodge, Steven

Hodgkinson, Colin

Hoerster, Katherine

Hoexter, Marcelo

Hoffman, Elizabeth

Hoffman, Ellen

Hoffman, Gabriel ...

Hoffman, Michael D.

Hofford, Rebecca

Hohmeister, Madeline

Hojlo, Margaret

Holem, Ryan

Hollander, Eric

Holloway, Ashley

Holly, Elizabeth

Holmes, Hollie

Holmes, Jacob

Holmes, Sophie

Holroyd, Kathryn

Holsen, Laura

Holt, Daphne

Holt, Emma

Holt, Leanne

Holton, Kristina

Holze, Friederike

Hommer, Rebecca

Honda, Shiori

Hong, Elliot

Hong, Sa-lk

Hong, Susie

Hong, Suzi

Hood, Caitlyn

Hooker, Jacob

Hopf, Frederic

Hopkins, Jordan

Hopkins, Seth

Hoppenstaed, Debra

Hoptman, Matthew J.

Horga, Guillermo

Horn, Paul

Horneffer, Yvonne

Horner, Michelle

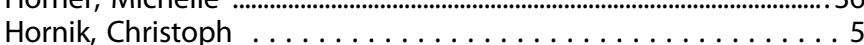

Horovitz, David ...............................................................................................

Hossein, Shabnam

Hough, Christina

Howard, David M.

P341

Howe, James R.

P778

Howe, William

P27

P194

Howell, Athena

P243

Howell, Paul

P58, P592, P668, P783

P149

P613

P118

P773

P126

P267

P507

P494

P685

P690
P785

P254, P370

P163

P726

P99
P44

... 49

P171

P461

P338

P615

P512

P126

P340

P787

P777

P464

P411

P186, P251, P357

P301, P342

P474

P824

P429
P579

579
586

P754

P196

P29

P380

P604

P641

P297

P286

P473
P173

P687
..P565, P816

36

P8

0

tos$$
\text { In }
$$$$
\text { Iosif }
$$

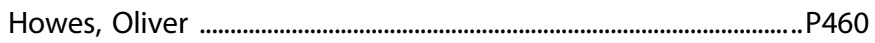

Howlett, Jonathon .......................................................................................... P55

Hsiao, Elaine .............................................................................................. 33

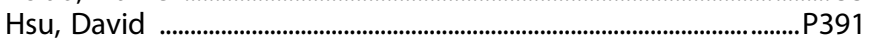

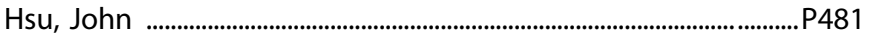

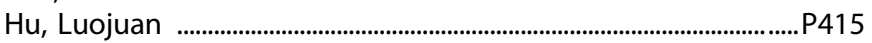

$\mathrm{Hu}$, Ruifeng ...................................................................................................P68

$\mathrm{Hu}$, Yang .........................................................................................................P197

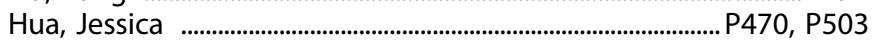

Huang, Grant .........................................................................................P477

Huang, Hailiang ................................................................................. P522, P798

Huang, Henry ................................................................. P58, P668, P766

Huang, LongJie .....................................................................................P750

Huang, Yanhua ..........................................................................P613, P739

Huang, Yiyun ..............................................................P210, P592, P783

Huang, Yung-Yu ........................................................................................... P264

Huang, Yuye ................................................................................................P746

Hubbard, Nicole ..............................................................................P607

Huber, Rebekah ................................................................................................ P2 P

Hudgens-Haney, Matthew .................................................................... P572

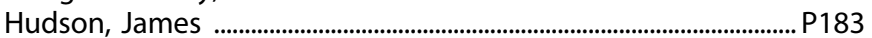

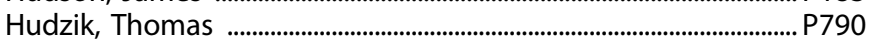

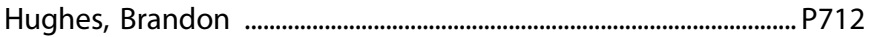

Hughes, Katelynn M. ...............................................................................P720

Hughes, Thomas ......................................................................................... P2

Hughes, Zoe .............................................................................. P235, P283

Huguet, Guillaume .............................................................................. P512

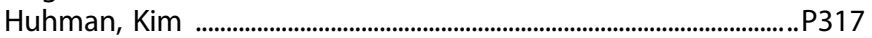

Huhn, Andrew .................................................................................................20.4

Hühne, Anisja .........................................................................................P771

Hultman, Christina ............................................................................ P445

Hunsberger, Holly .................................................................................... P7, P239

Hunter, Samantha ........................................................................................... P126

Huntley, George ........................................................................................... P2 265

Hurd, Yasmin .....................................................45, 45.4, P61, P674, P742

Hurowitz, Alexander ....................................................................................P128

Hurtado, Aura Maria ...............................................................P280, P286

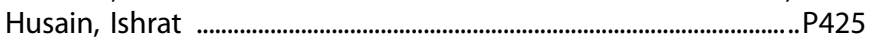

Husain, Mustafa ............................................................................................. 289, P406

Huskey, Alisa ..............................................................................................P370

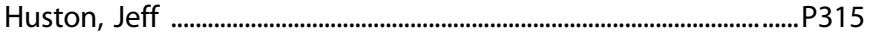

Hutchison, Kent ................................................................................................. P616

Hutten, Nadia ............................................................................................P824

Huuki, Louise ................................................................................................P2

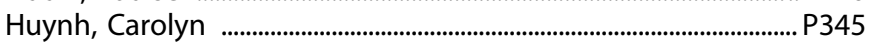

Hyde, Luke W. ............................................................................................... P140

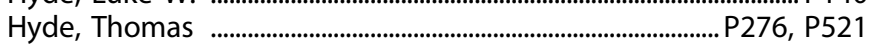

Hyer, Molly ....................................................................................................... P23

Hysek, Cedric ..................................................................................... P55

ladipaolo, Allesandra ................................................................................ P59

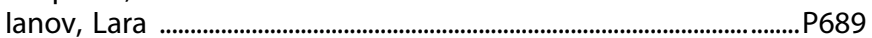

Ibarra, Christopher K. ............................................................................P768

Ibrahim, Mohamed ................................................................................. P649

lida, Madoka _..................................................................................P612

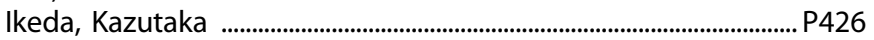

Ikuta, Toshikazu ....................................................................................... P821

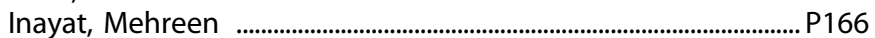

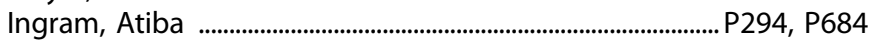

Inman, Jessica ......................................................................................P1 18

Inslicht, Sabra ........................................................................................... P42

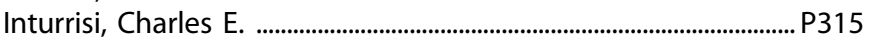

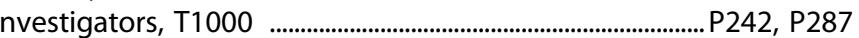

osif, Ana-Maria ............................................................................... P165, P543

osifescu, Dan .............................................................280, P286, P525

lqbal, Maisha ................................................................................................P153

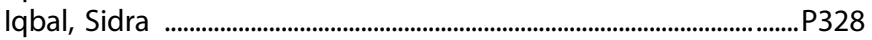

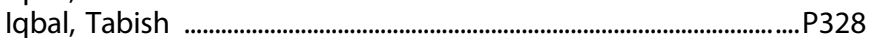

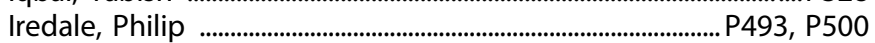


Irfan, Nadia

P310

Ironside, Maria

P242

Irvin, Molly

P280, P286

Irwin, Michael

$51.4, \mathrm{P} 482$

Ishikawa, Masago

P194

Ishimoto, Kenji ........................................................................................ P553

Ishizuka, Koko ........................................................................................P449

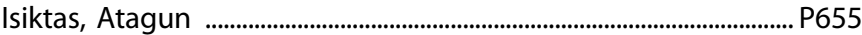

Ismail, Zahinoor

P10

Issler, Orna

P221, P301

Itoga, Christy A.

P387

Ivanov, Iliyan

P164

Ivleva, Elena

60.3

Ivy, Autumn

P108

Iwanaga, Dylan

P562, P576

Iwata, Yusuke

..P579

lyengar, Satish

P363

lyengar, Smriti

P420, P432

lyer, Eshaan S.

P347

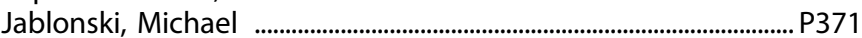

Jablonski, Monica ...................................................................................P196

Jackowski, Andrea .............................................................................. P149

jackson, Joshua ...................................................................................P637

Jacob, Suma .................................................................150, P154, P421

Jacobov, Rivky .....................................................................................P461

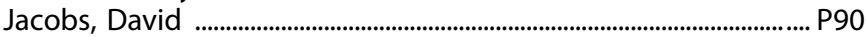

Jacobson, Mark .......................................................................................P246

Jacquemont, Sébastien ............................................................................... P512

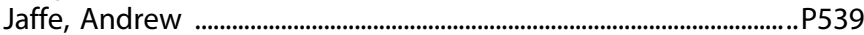

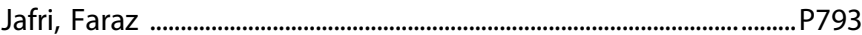

Jagasia, Puja ................................................................................................ P40

Jagasia, Ravi ........................................................................................... P56

Jain, Rakesh ..................................................................................................P319

James, Andrew .......................................................................................... P203

James, Anthony ................................................................................. P487

James, George …………………………………………………………..... P1 19

James, Morgan .............................................................................................P764

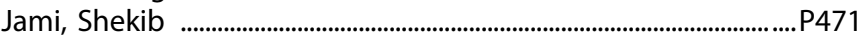

Janecka, Magdalena ........................................................................P120

Janes, Amy .......................................................................2475, P760, P769

Janowsky, David .................................................................................... P755

Jarcho, Johanna ................................................................................ P71

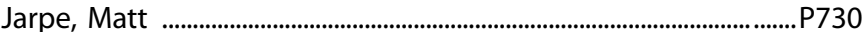

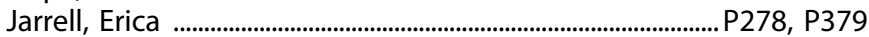

Jaster, Alaina ......................................................................................P627

Javanbakht, Arash .............................................................................................. P130

Javidfar, Behnam ....................................................................................P744

Javitch, Jonathan …................................................. P207, P292

Javitt, Daniel ................................................................P458, P527, P574

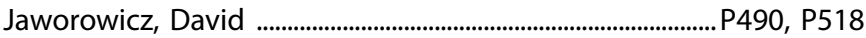

Jaworska, Natalia ...................................................................................... P153

Jean-Louis, Alessandro ............................................................................. P684

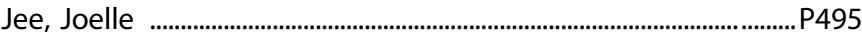

Jenkins, Lisanne ........................................................................................ P791

Jennings, Mariela ........................................................................................ P746

Jennings Mathis, Karen .......................................................................... P44

Jensen, Lars Thorbjørn ....................................................................P526

Jentsch, James …………………………………….....42, P199, P714

Jessing, Kevin ...……………………………………………………………. P338

Jeste, Dilip ...................................................................................... 33, P482

Jeste, Shafali ....................................................................................5, P187

Jha, Manish ........................................47, P1, P20, P68, P241, P394, P674

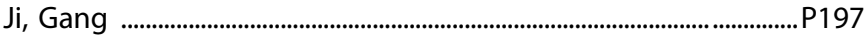

Jia, Caroline ..................................................................................................P267

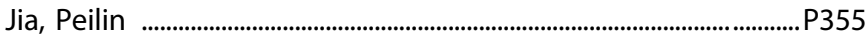

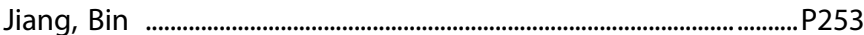

Jiang, Chenguang ................................................................................ P522
Jiang, Yan

P461

Jimenez, Esther ................................................................................... P17

Jimenez, Jessica ................................................................................. P331

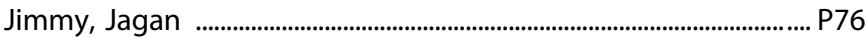

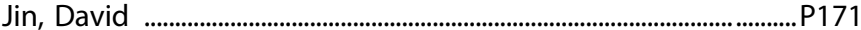

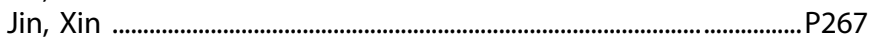

Jinadasa, Tushare ....................................................................................... P331

Jindrová, Miroslava ............................................................................ P447

Jo, Young Chan .......................................................................................... P345

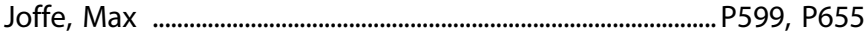

Johannesen, Jason K ................................................................................. P545

John, Majnu .................................................................................. P506, P516

Johnson, Allison …………………………………........ 6679, P699, P709

Johnson, Amy ..............................................................................................P629

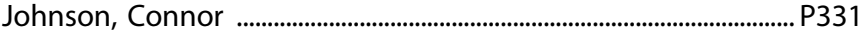

Johnson, Emma .................................................................................. 13.4, P184

Johnson, Matthew ................................................................................... P654

Johnson, Michael .................................................................................. P634

Johnson, Paul ...................................................................................................P194

Johnson, Shane ........................................................................................ P213

Johnson, Sheri L. ................................................................................................ P304

Johnson-Pais, Teresa ............................................................................... P12

Johnston, Camille ................................................................................... P71

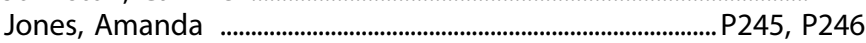

Jones, Sara ..........................................................................................P650

Joo, Yoonjung Yoonie .............................................................................. P132

Jordan, Ayana .................................................................................................... 12

Jordan, Chloe ........................................................................................P728

Jordan, Kelley ...................................................................................................

Jørgensen, Mathias ............................................................................... P542

Joseph, Dieuseul ......................................................................... P684

Joseph, Paule ....................................................................P678, P680, P700

Joshi, Sonalee ..........................................................................P748, P801

Joshi, Yash ....................................P25, P476, P477, P573, P576, P827

Josselyn, Sheena ........................................................................................... P49

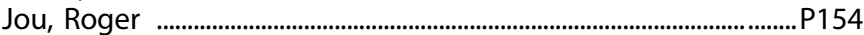

Jouroukhin, Yan ................................................................................................ P558

Jovanovic, Tanja .......... P48, P53, P72, P79, P110, P130, P170, P788

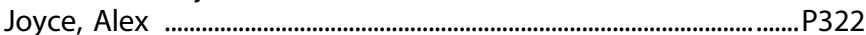

Juarez, Barbara ............................................................................................ P177

Jung, Jeesun .........................................................................................P778

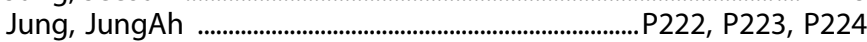

Jungaberle, Andrea .................................................................................P350

Jungaberle, Henrik .....................................................................................P350

Jurewicz, Katarzyna ................................................................................ P792

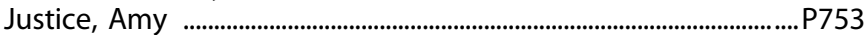

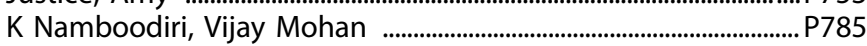

Kaar, Stephen ..........................................................................................P460

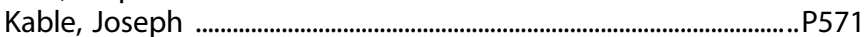

Kaddurah-Daouk, Rima ............................................................................ P349

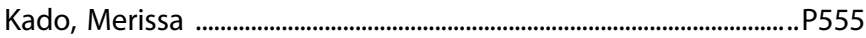

Kadriu, Bashkim ...................................................................................... P2 P

Kahlig, Kristopher ................................................................................... P283

Kahn, Charles ………………………………………………………....P568

Kahn, Rene ...................................................................................................... 19

Kaiser, Roselinde ...................................................................... P341, P389

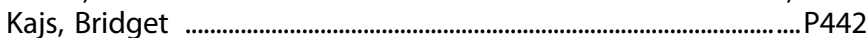

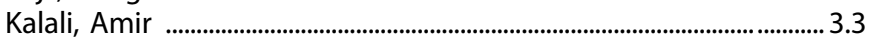

Kalemba, Katarzyna ................................................................................... P757

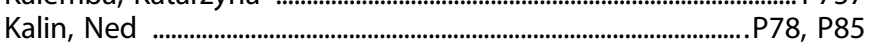

Kalivas, Peter ............................................................ P67, P615, P628, P717

Kallioniemi, Elisa .............................................................................. P215

Kalluchi, Achyuth ............................................................................................. P80

Kallupi, Marsida _............................................................2677, P701, P759

Kamarajan, Chella ................................................................................ P184

Kamath, Rahil ..........................................................................................P325

Kamei, Noriko .................................................................................P665

Kamilar-Britt, Philip ...................................................................................... P674, P807 
Kaminsky, Jesse ................................................................................. P213

Kaminsky, Zachary ...................................................................................P778

Kamiya, Atsushi ...............................................................................P558

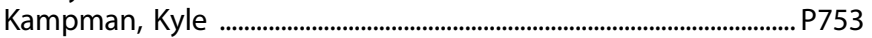

Kandel, Eric

P759

Kane, John

$19,60, \mathrm{P} 493, \mathrm{P} 516, \mathrm{P} 541$

Kanekar, Shami

10, P338

Kanen, Jonathan

Kanes, Stephen J

Kang, Hakmook

Kang, Seungwoo

Kang, Shinwoo

Kang, Sun

Kanne, Stephen

Kano, Shin-ichi

Kantrowitz, Joshua

Kapinos, Michael

$\mathrm{P} 800$

P222, P223, P224, P271

P368

P662, P754

P754

P121

P126

P24, P830

Kaplan, Sydney

Karbalaei, Reza

Kareken, David

Karim, Helmet

Karlsson Linner, Richard

Karmacharya, Rakesh

Karoly, Hollis

Karp, Barbara I.

Karp, Jordan

Karpyak, Victor

Kash, Thomas

Kasper, Siegfried

Kassem, Layla

Kassim, Bibi

Kassir, Suham

Kato, Hiroyuki

Katz, Daniel

Kaundinya, Gopinath

Kautzky, Alexander

Kavelaars, Annemieke

Kaybay, Kayhan

Kaye, Walter

Kazan, Joseph

Kearney-Ramos, Tonisha

Kearns, Angela

Keck, Thomas

Keding, Taylor

Kee, Noelle

Keedy, Sarah

Kegeles, Lawrence

Keiser, Ashley

Keisler, Maria N.

Kellendonk, Christoph

Kelley, Lisalynn

Kellman, Mia

Kellner, Charles

Kelly, Deanna

Kelly, Erin

Kelmendi, Benjamin

Kelsoe, John

Kember, Rachel

Kendler, Kenneth

Kendzior, Brian

Kennedy, David

Kennedy, James

Kenny, Paul

Kenton, Johnny

Kenwood, Margaux

Keppler, Lydia

Kerr, Tony

Keshavan, Matcheri
P458, P525, P574

P668

P168

P294, P684, P737

P707

P32, P74, P825

P703

P151, P504

P616

P429

8.2, P231

P762

P417, P645, P683

P240

P123

P461

..P259

..P39

P178

P112

P240

... P5

P649

P116

. P11

55.3

P641, P712

P590

P167

... P60

P503, P572

P458

P3

P27

P207, P534, P537

P388, P789

..P421

... P406

33, P569

P633

15

P303

P753, P819

P226

P631

P163

P146

P194, P601

P815

47, P78

P66

P591, P681

P487, P565, P572
Ketchesin, Kyle

P532

Kevin, Garey

P334

Keyes, Laurel

Keynan, Jackob

P267

Keyser-Marcus, Lori

P447

Khachadourian, Vahe ..............................................................................P120

Khalil, Huzefa ...............................................................................P269, P336

Khalsa, Sahib .....................................................................................P209

Khan, Arshad ...............................................................................................P714

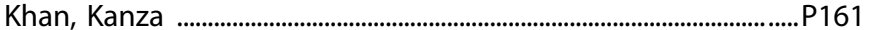

Khanipov, Kamil ................................................................................................ P6 636

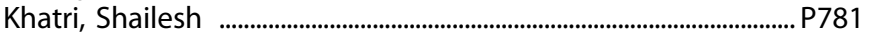

Khom, Sophia …….........................................................................................P681

Khorrami, Kia ................................................................................................P185

Khoury, Talah .............................................................................................P205

Khuder, Sadik ...................................................................................................P550

Kigar, Stacey ...................................................................................................P329

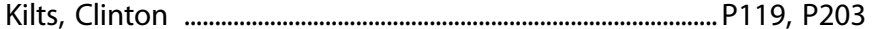

Kiluk, Brian ......................................................................................................P749

Kim, Angela ...................................................................................................P195

Kim, Diane .................................................................................................P313

Kim, Hannah .....................................................................................................P687

Kim, Hyun-Jin .....................................................................................

Kim, Hyunjung ....................................................................................... P662

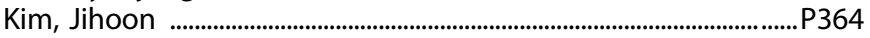

Kim, Juhyun ......................................................................................................P558

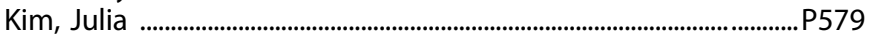

Kim, Julia Minjung ......................................................................................P808

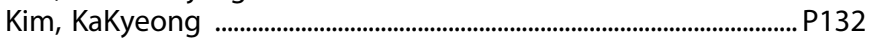

Kim, Kelly .....................................................................................................P672

Kim, Kongpyung .................................................................................................. P578

Kim, Michelle .................................................................................................P647

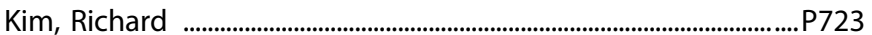

Kim, Ronald .....................................................................................................P256

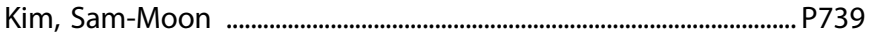

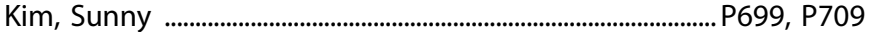

Kimbrough, Adam .................................................................................. P677

Kimmel, Mary ...................................................................................................53.2

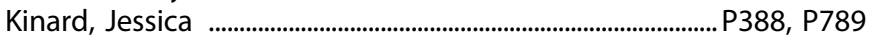

King, Andrea ...........................................................................17, 17.3

King, Christopher ......................................................................................P705

King, Raven ............................................................................................P713

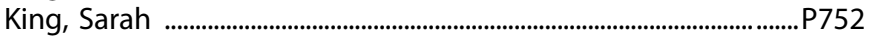

Kingsbury, Marcy ......................................................................................P129

Kinkead, Becky ....................................................................................... P361

Kinnaird, Brian ...................................................................................... P262

Kinreich, Sivan ....................................................................................................... P184

Kious, Brent ...............................................................................................P281

Kippenhan, J. Shane ....................................................................P107, P147

Kiraly, Drew ....................................................................P647, P715, P740

Kircanski, Katharina ……………….......................... P37, P403, P799

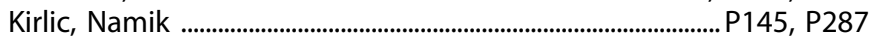

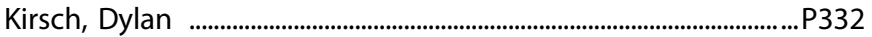

Kish, Stephen ..............................................................................................P767

Kitamura, Takashi ....................................................................................... P528

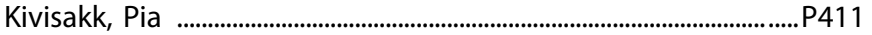

Kivlichan, Ashley .................................................................................P179

Klei, Lambertus ........................................................................................ P445

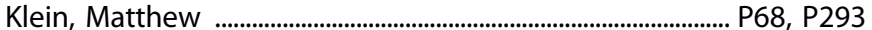

Kleinman, Joel .................................................................... P93, P276, P521

Klengel, Claudia ...................................................................................... P290

Klengel, Torsten ...................................................... P96, P236, P278, P290

Klimes-Dougan, Bonnie ............................................................................ P817

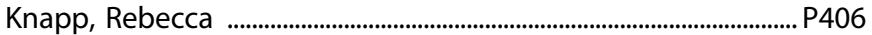

Knickmeyer, Rebecca ...................................................................................... P795

Knippenberg, Anna .................................................................................. P66

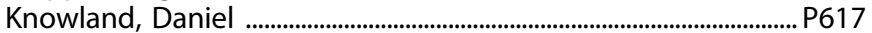

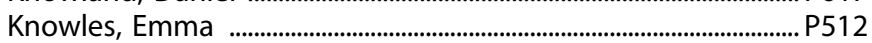


Kobayashi, Kazuto

Koblan, Kenneth

Kochoian, Brik .

Kochunov, Peter

Kodesh, Arad

Koek, Wouter

Koenigsberg, Harold

Kohler, Christer

Kohler, Christian

Kohn, Jordan

Kohn, Philip

Koike, Hiroyuki

Kolachana, Bhaskar

Kolb, Hartmuth

Kolla, Nathan

Kollins, Scott

Kondev, Veronika

Kondo, Douglas

Konishi, Kyoko

Kononoff, Jenni

Konova, Anna

Koob, George

Kooiker, Cassandra

Kopelman, Jared

Korankyi, Ivana .

Kosaraju, Siddartha

Kosheleff, Alisa

Koshimori, Yuko

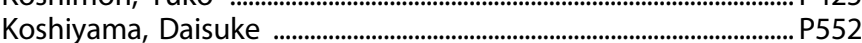

Koslowski, Michael .............................................................................P350

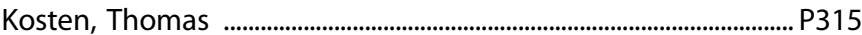

Kotsouris, Anastasia ................................................................................... P369

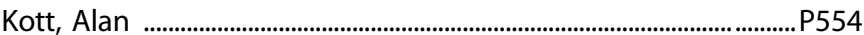

Kotz, Juliana ...................................................................... P25, P562, P576

Kouchakidivkolaei, Maryam ................................................................. P791

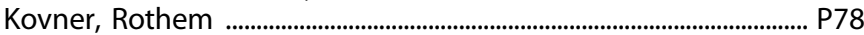

Kowalchuk, Chantel .............................................................................. P559

Kozak, Karolina ......................................................................................P502

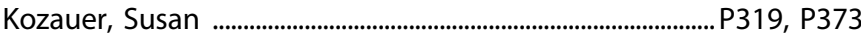

Kozhemiako, Nataliia ........................................................................P522

Kozicz, Tamas .............................................................................................P302

Kozlenkov, Alexej ................................................................................... P461

Krafty, Robert ............................................................................................. P74

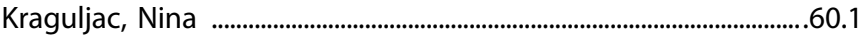

Krajmalnik-Brown, Rosa ........................................................................... 33

Krall, Mckenna ............................................................................................. P495

Kramár, Enikö .......................................................................................................

Kramer, Edgar ..........................................................................................P446

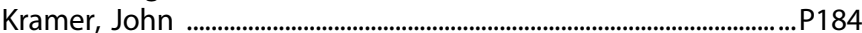

Kranzler, Henry .................................................................P630, P753, P819

Kraus, Christoph ................................................................................... P240

Kreich, Mike ..................................................................................................P28

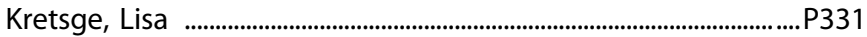

Krienen, Fenna .................................................................................. P236

Krishnan-Sarin, Suchitra ..........................................................................P766

Kroll, Danielle ....................................................................679, P699, P709

Kronberg, Greg …………………………………………………………...... P625

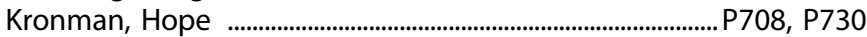

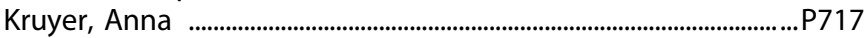

Krystal, Andrew .......................................................................................... P581

Krystal, John .............................................................P61, P94, P493, P783

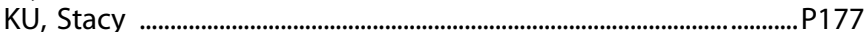

Kubicki, Marek ........................................................................... P487, P565

Kudinova, Anastacia ............................................................................ P812

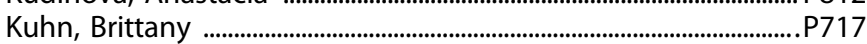

Kulick-Soper, Colin ................................................................................... P607

Kumakura, Yoshitaka ..............................................................................P526

Kumar, Poornima ......................................................................................... P325
Kumar, Sanjeev

P314

Kummerfeld, Erich

P150, P632

Kundakovic, Marija

P80, P375, P461

Kundu, Ashima .....................................................................................P387

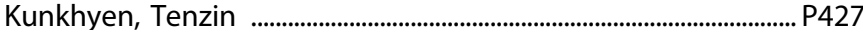

Kupferschmidt, David ..............................................................................P459

Kuplicki, Rayus ....................................................P209, P242, P287, P652

Kura, Nikki

Kuret, Thomas _.......................................................................................... P303

Kushner, Matt ..........................................................................................P605

Kutlu, Munir .....................................................................................................P655

Kuypers, Kim ...........................................................................................P824

Kvarta, Mark ...................................................................................P456

Kwako, Laura ...............................................................................................P687

Kwon, Wooseok ...................................................................................P529

Kyzar, Evan ......................................................................................................P747

La Via, Luca ........................................................................................ P87

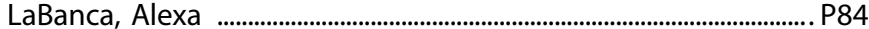

Lacadie, Cheryl ................................................................................................ P804

Lacagnina, Anthony ............................................................................... P252

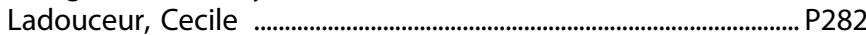

Laezza, Fernanda ................................................................................... P308

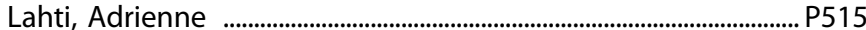

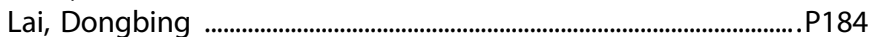

Lai, Meng-Chuan .................................................................................. P418

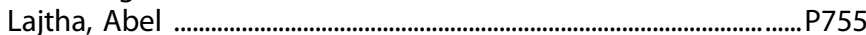

Lal, Asim .................................................................................................P310

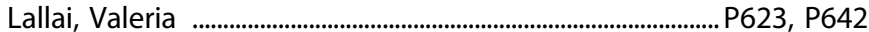

Lalush, David …………………………………………………............ P388, P789

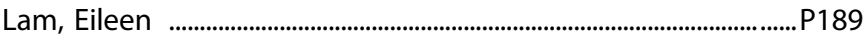

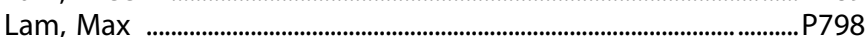

Lam, Sherry ................................................................................................P233

Lam, TuKiet ............................................................................................P647

Lambe, Evelyn ....................................................................................... P4 P

Lambert, Jeremy .......................................................................................... P114

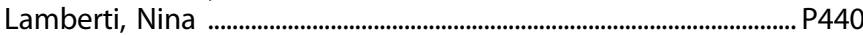

Lamon-Fava, Stefania ............................................................................... P361

Lancaster, Eva .........................................................................................P226

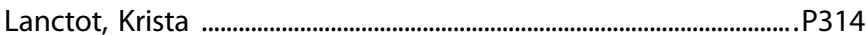

Land, Benjamin ....................................................................................... P779

Landavazo, Antonio .............................................................................P192

Landen, Mikael .......................................................................................... P445

Landi, Isotta ..................................................................................................... P62

Landman, Bennett ....................................................................................... P368

Lane, J. Robert .................................................................................................. P547

Langenecker, Scott .................................................................P115, P261

Langfus, Joshua ………………………………………................... P200, P202, P238

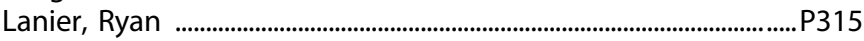

Lanio, Marcos ............................................................................................. P60

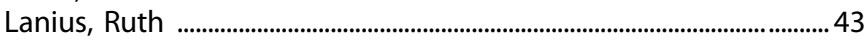

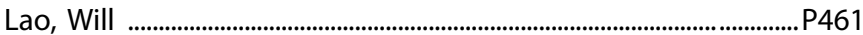

Lapish, Christopher ................................................................................ P604

Lardenoije, Roy ......................................................................................... P96

Large, Charles ...........................................................................................P460

Larsen, Bart ............................................................................................................ P148

Larsson, Evelina ....................................................................................... P696

Lasser, Robert .....................................................P2222, P223, P224, P271

Laukkanen, Virpi .....................................................................................P215

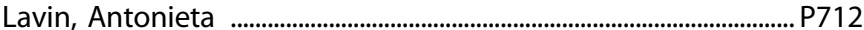

Lavretsky, Helen ...............................................................8, 8.3, P227, P231

Law, Rebecca ...................................................................................................P371

Law, Stephanie …......................................................................................... P1 14

Lawrence, Ryan ................................................................................................. 32

Laws, Keith .....................................................................................P434

Lawson, Elizabeth ........................................................................................... P186

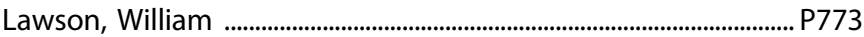

Lazarevic, Vesna .................................................................................. P400

Le, Quan-son .............................................................................................. P89 


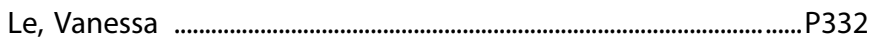

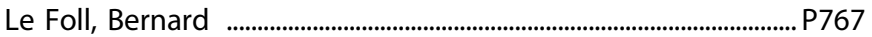

Leach, Robin .......................................................................................... P12

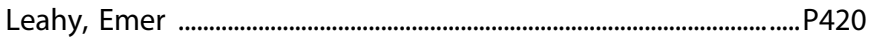

Leal, Juan Andrew .............................................................................. P29

Lebayle, Ekaterina ................................................................................... P750

LeBois, Lauren ............................................................................................. P78

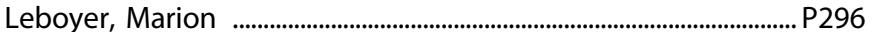

Lee, Beth

Lee, Brian

P678, P680, P700

Lee, Chris

$P 27, P 672$

Lee, Ellen

$\mathrm{P} 83, \mathrm{P} 482$

Lee, Eun-Ji

P30, P269

Lee, Francis

.........P413

Lee, Jeong

P527

Lee, Jeyeon

P662, P754

Lee, Jisoo

P778

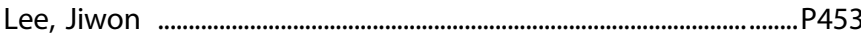

Lee, Junghee ...............................................................................................P515

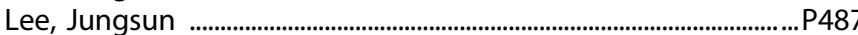

Lee, Ka Shu ..............................................................................................P797

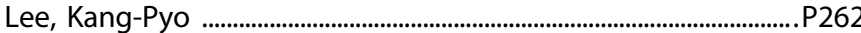

Lee, Kyunghun ......................................................................................... P37

Lee, Minyoung ........................................................................................ P811

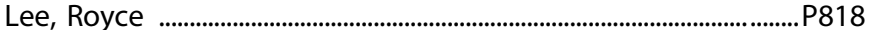

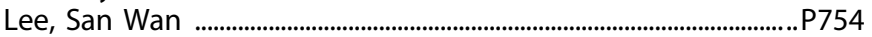

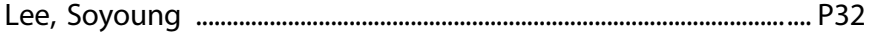

Lee, Virginia .....................................................................................................P664

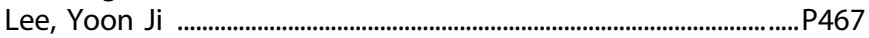

Legarreta, Margaret ........................................................................... P34

Leggio, Lorenzo ...............................................................P648, P726, P741

Lehman, Julia .......................................................................................P201

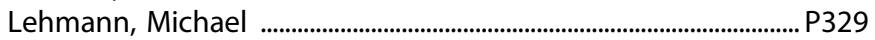

Lehmann, Vanessa

Lei, $\mathrm{Du}$

P397, P398, P399

Leibenluft, Ellen

Lemieux, Jacob E.

Lemieux, MacKenzie

Lemke, Hannah

Lenartowicz, Ewelina

Lencz, Todd

Lengvenyte, Aiste

Lenroot, Rhoshel

Lenze, Eric

Leonard, Michael

Leonardo, E. David

Leong, Kam .

Leoni, Matthew

Leopold, David

Lepeak, Lauren

Lepow, Lauren

Leptak, Chris

Lerch, Jason

Lerer, Bernard

Lerer, Leonard

Lerman, Caryn

Lerner, Talia

Lerou, Paul

Lesh, Tyler

Lesiak, Atom

Lessie, Erin

Lesuis, Sylvie

Levi, Linda

Levine, Stephen

47, P37, P214, P403, P797, P799

P411

P27, P267

P569

P691

P463

P455, P506, P540, P798

P296

P454

... 8, 8.1, P231, P354

P635

P446

P264

P493, P500

P527

P649

P62, P164

... 36

P463

2, P181, P365

P181, P365

P760

P340

P151

Levinson, Tess

Levis, Sophia
Levison, Julie

P151

Levitt, James

P565

Levy, Emily

P266

Lewis, Alan

P529

Lewis, Ben

P610

Lewis, Charles

P144

Lewis, David

Lewis, Michael

Lewis-de los Angeles, William

P371, P468, P484, P532, P549, P567, P765

eyton, Marco …

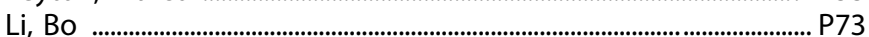

Li, Chiang-shan ...................................................................................... P780

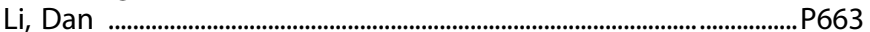

Li, Dongyang ................................................................................................P189

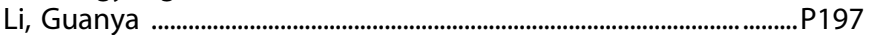

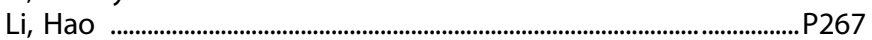

Li, Hongyu ............................................................................................. P94

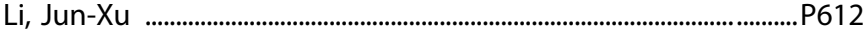

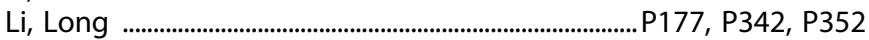

Li, Ningshan ...............................................................................................P171

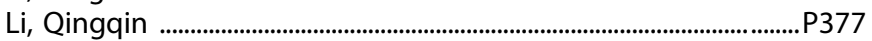

Li, Yan .............................................................................................. P195, P621

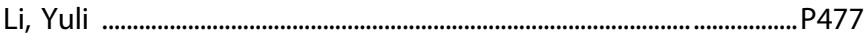

Li, Yulong ............................................................................. 30.3, P207, P267

LI, Zhihao ................................................................................................ P64

Liang, Katharine .................................................................................................. P45

Liang, Winnie ................................................................................................P634

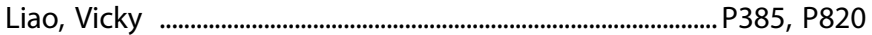

Liberman, Mark .................................................................................................. P496

Libowitz, Mark ............................................................................................. P695

Libster, Avraham ................................................................................... P267

Lichenstein, Sarah .................................................................................. P749

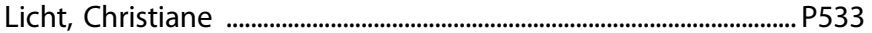

Licinio, Julio .......................................................................................................P396

Lidstone, Daniel ........................................................................................... P784

Lieberman, Jeffrey ......................................................................P525, P566

Liechti, Matthias ......................................................................................... P824

Liew, Zeyan ..............................................................................................P797

Light, Gregory ..........................................P25, P476, P477, P545, P552,

P562, P573, P576, P827

Lijffijt, Marijn

Lim, Kelvin ...

Lim, Sangtaeck

P247, P421, P605, P632

Lima, Camila Nayane de Carvalho $\quad$ P275, P362

Lin, Alexander

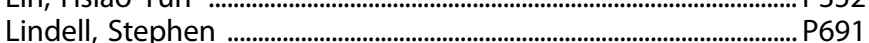

Lindenmaier, Zsuzsa ................................................................................P463

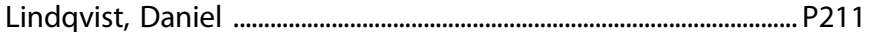

Ling, Emi .....................................................................................................P236

Linguiti, Sophia ………………………………………........................... P214

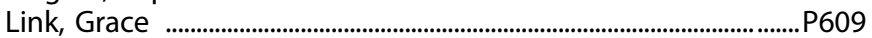

Linke, Julia ................................................................................... P43, P799

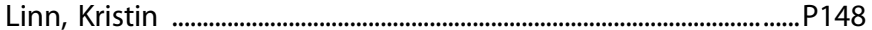

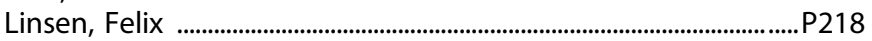

Lippard, Elizabeth .................................................................................. P332

Lipschitz, Jessica ................................................................................ P228

Lisa, von Moltke ...................................................................................... P334

Lisanby, Sarah ................................................................. P289, P406

Liss, Thomas ..............................................................................................P639

Liston, Conor ............................................................................... 25.2, P213

Litif, Carl ........................................................................................................P772

Litman, Robert ..............................................................................P272, P514

Liu, Chunyu ......................................................................38, 38.4, P829

Liu, Fred ........................................................................................................ P8

Liu, Hesheng ..............................................................................................P201

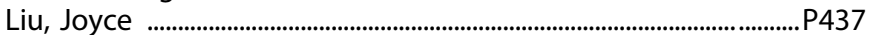

Liu, Loretta ......................................................................................................P109 
Liu, Qing-song

Liu, Rui .....................

P813

P428

Sky

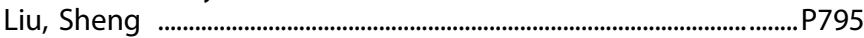

Liu, Wenzhong ................................................................................... P146

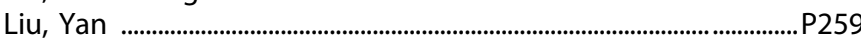

Liu, Yang .........................................................................................................P264

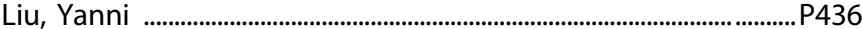

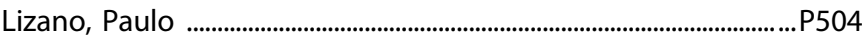

Lobo, Judith D.

P29

Lobo, Mary Kay ............................................................................P598, P612

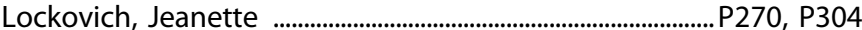

Lodge, Daniel ........................................................................... P6489

Loebel, Antony ...................................................................................... P491

Loewy, Rachel .............................................................................................P555

Logan, Ryan ................................................................................P360, P765

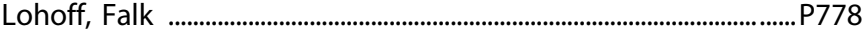

Lolli, Bridget .........................................................................................................P126

Lombroso, Sonia .........................................................................................P664

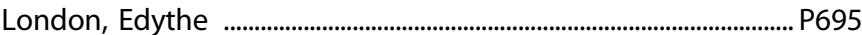

Loo, Sandra ................................................................................................ 9.1

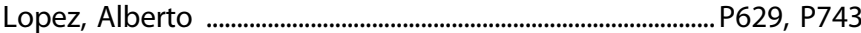

Lopez, Joëlle .............................................................................................P295

Lopez, Juan ............................................................................................P336

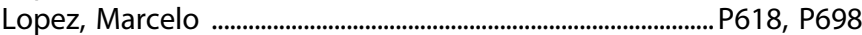

Lopez, Savannah ................................................................................. P41

Lopez-Duran, Nestor .................................................................................. P140

Lopez-Guzman, Silvia ................................................................................. P58

Lopez-Quintero, Catalina .....................................................................P587

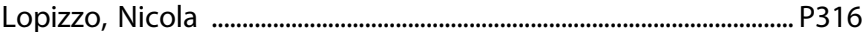

Lopresti, Brian .................................................................................................. P409

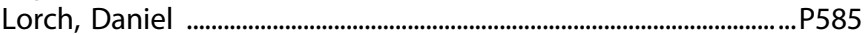

Lorsch, Zachary .........................................................................................P301

Loughead, James .......................................................................................... P760

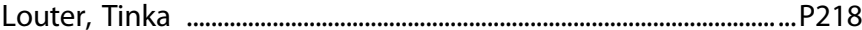

Love, Tiffany ............................................................................................P819

Lovinger, David ........................................................................................... P413

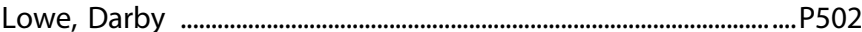

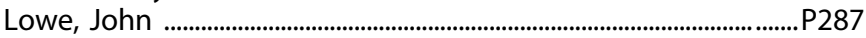

Lowes, Daniel ........................................................................ 128, P216

Luby, Joan .......................................................................... P28, P135, P168

Luchsinger, Joseph ..................................................................................... P86

Luck, Steven ....................................................................................P551

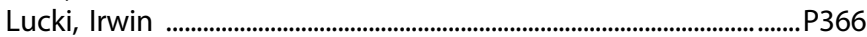

Lueptow, Lindsay ...................................................................................P608

Lugo-Candelas, Claudia .................................................................... P149

Luk, Jeremy ............................................................P678, P680, P687, P700

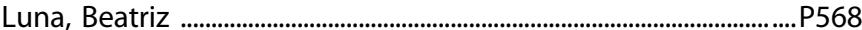

Lund, Johan ............................................................................................P572

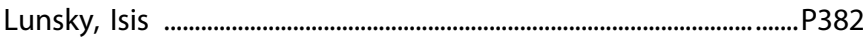

Luo, Jiajun .................................................................................................P797

Luo, Qiang .....................................................................................................P800

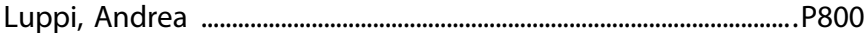

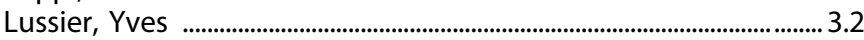

Lustberg, Daniel .....................................................................................P437

Luthringer, Remy ........................................................................................... P546

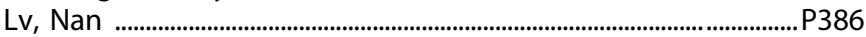

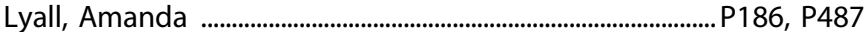

Lydon-Staley, David ........................................................................................ P2 14

Lynch, William B. .................................................................................. P735

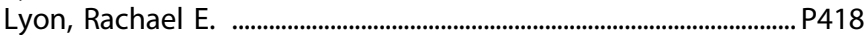

Lyu, Ilwoo .......................................................................................................P486

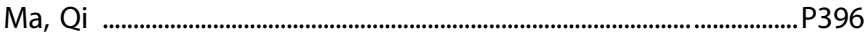

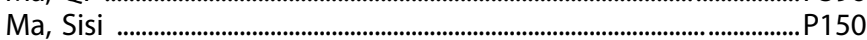

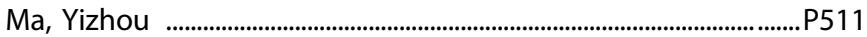

Määttä, Sara .....................................................................................P215

Macaluso, Matthew .............................................................................. P371
MacDonald, Angus

P511

MacDonald, Laura

$P 573, P 827$

MacDonald, Matthew

$P 449, P 549, P 765$

MacKillop, James ..................................................................................P695

MacNamara, Annmarie ............................................................ 37.3, P786

Macpherson, Tom .................................................................................... P823

Maddock, Richard ....................................................................... P165, P543

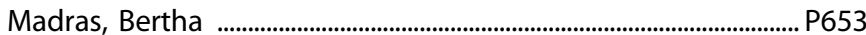

Maguire, David .......................................................................................P718

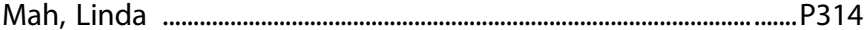

Mahalingam, Rajasekaran ............................................................................... P5

Mahant, Iteeben .............................................................................................

Mahbubani, Rebecca ............................................................................ P32

Mahe, Karan .......................................................................................P779

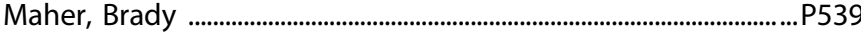

Maher, Erin .......................................................................................................P781

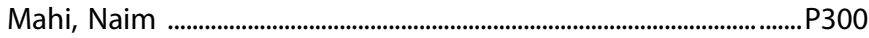

Mahjani, Behrang ……………………………………………............. P445

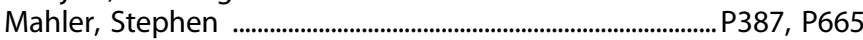

Mahon, Pamela ...........................................................................P385, P820

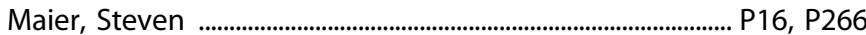

Majewska, Barine ................................................................................. P654

Majić, Tomislav ......................................................................................... P350

Makary, Meena ........................................................................................... P631

Makhoul, Walid ......................................................................................... P392

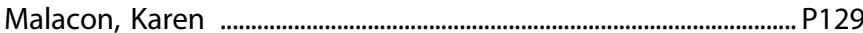

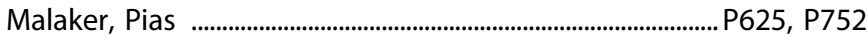

Malaspina, Dolores ......................................................................................... 12

Malcolm, Robert ................................................................................................ P6

Malgady, Jeffrey ....................................................................................... P535

Malhotra, Anil ............................... P455, P469, P487, P506, P810, P821

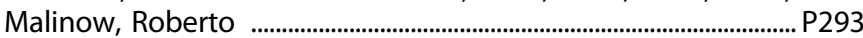

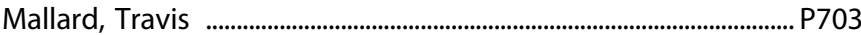

Mammo, Dalia .................................................................................... P130

Manduca, Joshua ....................................................................................... P356

Manea, Ana .....................P277

Manfredi, Paolo L.................................................................................... P315

Manhart, Megan ............................................................................................. P28

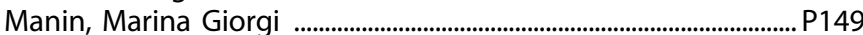

Mann, J. John .....................................................................P259, P264, P448

Männel, Claudia ......................................................................................P141

Manning, Elizabeth ............................................................................... P4 P48

Manoach, Dara .......................................................................................... P522

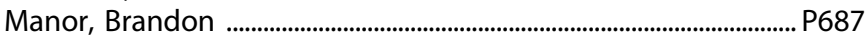

Manthena, Akhila .................................................................................. P757

Mantsch, John ............................................................................................... P722

Mantua, Valentina . . . . . . . . . . . . . . . . . . . . . . 5

Manvich, Daniel ........................................................................................P607

Manza, Peter .............................. P197, P285, P679, P692, P699, P709

Manzano, Lawrence ................................................................................... P611

Manzler, Charles ........................................................................................... P185

Manzor Mitrzyk, Beatriz ............................................................................. P333

Maples-Keller, Jessica ....................................................................... P18, P70

Mar, Wanna .......................................................................................P579

Marchisella, Francesca .................................................................................... P320

Marcinkiewcz, Catherine .................................................56, 56.2, P161

Marcotte, Thomas …………………………………………………....... P755

Marder, Stephen .................................................................. 19, 32, P491

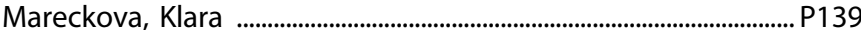

Marenco, Stefano ........................................................................................... P461

Margetts, Alexander ..........................................................................P624

Margolin, David ......................................................................................... P5 P

Margolis, Amy ........................................................................................ 44, 44.4

Maria Rios, Cristina _........................................................................P626

Marin, Frances ............................................................................................... P605

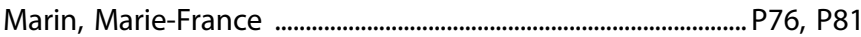

Marizzoni, Moira ……………………………………………………….... P316

Marjanska, Malgorzata .................................................................................. P483 
Marks, William

P528

Markx, Sander

P566

Maron-Katz, Adi

P176

Marquardt, Ashley

P117

Marques, Ana

P75

Marrocco, Gabriel

P792

Marrocco, Jordan

Marsh, Rachel

Marshall, Wyatt

24, 58, P269

Martin, Caitlin ..

26, 26.3, P149, P822

Martin, Melissa

P530

P583

Martin Fardon, Rémi ..............................................................................P681

Martinez, Angelica ...............................................................................P677

Martinez, Kimberly .............................................................................. P57

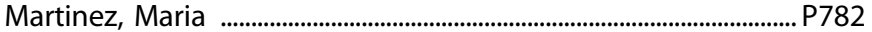

Martinez, Suky .................................................................................... P790

Martinez, Vena ..............................................................................................P5

Martinez-Rivera, Freddyson

P657

Martínez-Rivera, Freddyson J.

P221, P342

Martinowich, Keri

29.3, P539

Martins-Junior, Ronaldo

P424

Martis, Brian

P83

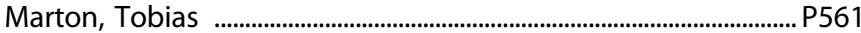

Martyniuk, Kelly ....................................................................................... P2 207

Maruff, Paul ................................................................................................. P51

Marugan, Juan ..................................................................................................... P547

Marusak, Hilary ...................................................................................... P111

Marvin, Morgan ................................................................................................. P88

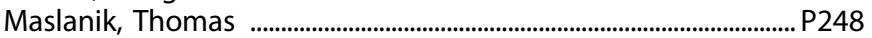

Mason, Natasha ...........................................................................................P824

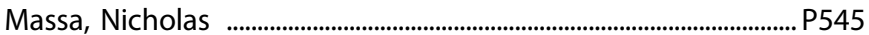

Mastro, Kevin ........................................................................................ P13

Mastrobattista, Emma ............................................................................P354

Mastrodonato, Alessia ......................................................................... P60

Masutani, Teruaki ..................................................................................... P553

Mataix-Cols, David ................................................................................... P445

Mateo, Yolanda ........................................................................................ P413

Matera, Carlo ...................................................................................................P642

Mates, Sharon

Mathalon, Daniel

P319, P373

Mathe, Aleksander ........................................................................ P254

Matheos, Dina ........................................................................................................

Mathew, Sanjay ........................................................................................ P328

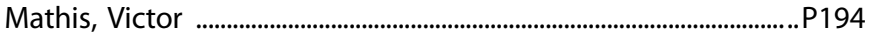

Mathur, Brian ..............................................................................2327, P814

Matoba, Ken .............................................................................. P24, P830

Matta, Jose ....................................................................................................P6

Mattheisen, Manuel ................................................................................... P445

Matthews, Lindsay ......................................................................................... P555

Matthews, Marguerite .................................................................................... 40

Mattingly, Gregory W .......................................................................... P271

Maturin, Lisa

P677, P701

Matuskey, David

P592, P668

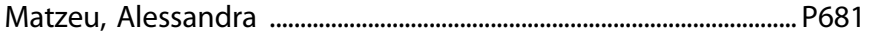

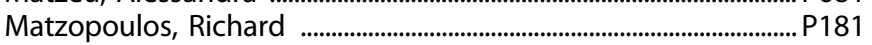

Maurer, Sara .......................................................................................... P1

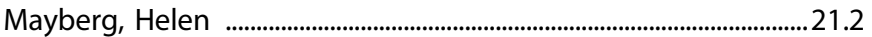

Mayeli, Ahmad ........................................................................................... P538

Mayer, Cynthia ............................................................................................P777

Mayer, Grace

P454

Mayes, Taryn

Mayfield, R. Dayne

Maynard, Kristen

Mayo, Leah

Maze, lan

Mazurek, Micah

Mazurka, Raegan

Mazzelli, Monica

P1, P349, P394

P611, P698

P276

P696, P771

P417

P126

P696, P771

P316
Mazzu, Maria

McAdams, Carrie

McAllister, A. Kimberley

P165, P543

McAlpin, Blake .................................................................................................P5

McCabe, Connor ............................................................................. P763

McCall, Nora ............................................................................. 42.2, P339

McCall, W. Vaughn .................................................................................... P406

McCalle, Catherine .......................................................................... P99

McCane, Aqilah .......................................................................................P702

McCann, Katharine ...................................................................................P437

McCarroll, Steve .................................................................................. P236

McCarthy, Julie ......................................................................................... P556

McCarthy, Margaret

4.1, P117, P327

McCarthy, Michael

P383, P401

McCauley, Bryan ........................................................................................... P375

McClain, Natalie ................................................................................................ P625

McClellan, Emily ..................................................................................... P495

McClintock, Shawn .....................................................P289, P374, P406

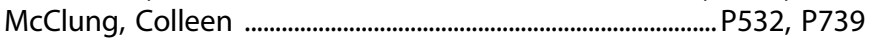

McClure, Margaret .......................................................................................P210

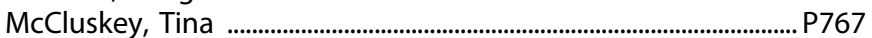

McCullough, Kenneth .............................................P35, P95, P290, P379

McCullumsmith, Robert ..............................................P322, P550, P559

McCurdy, Christopher ............................................................................... P667

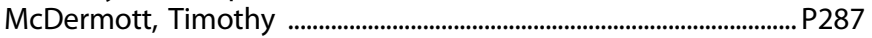

McDonald, Stephany ….............................................................................. P607

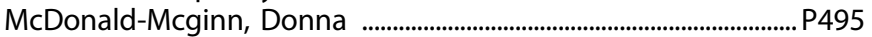

McDougle, Christopher ........................................................... 16, P416

McDuffie, Christian ........................................................................................ P680

McElligott, Zoe ..............................................................................................56.4

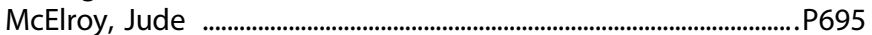

McElroy, Susan ................................................................................................. P302

McEwen, Bruce .................................................................................... P269

McGinn, Daniel ............................................................................................. P495

McGinty, Jacqueline ............................................................................45, 45.2

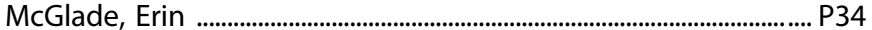

McGlashan, Thomas H .............................................................................. P545

McGowan, Josephine .................................................................................. P50

McHugo, Maureen .................................................................................P486

Mclnnis, Melvin ............................................................................P288, P298

Mclntosh, Andrew M ........................................................................P778

Mclntosh, Scot ..................................................................................... P590

Mclntyre, Roger ................................................................................. P319

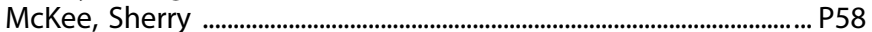

McKinzie, David ............................................................................................. P510

McLean, Samuel ........................................................................................ P788

McLoyd, Vonnie C. ....................................................................................... P140

McMahon, Francis ................................................................................... P123

McNally, Gavan .................................22.4

McNamara, Amanda ............................................................................... P55

McNamara, Margaret .......................................................................... P178

McNamara, Nora ........................................................................................ P154

McNamara, Robert ........................................................P397, P398, P399

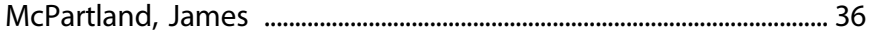

McPherson, Katherine ..................................................P679, P699, P709

McQuail, Joseph ............................................................................................P8

McReynolds, Jayme .............................................................................P722

McTigue, Anna ....................................................................................... P809

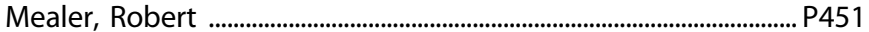

Meaney, Michael J. .......................................................................P254, P299

Meckel, Katherine ....................................................... 647, P715, P740

Medalia, Alice ...........................................................................................P525

Medeiros, Destynie ............................................................................... P830

Meggan, Drennan ....................................................................................P616

Meghani, Salimah ....................................................................................... P63

Mehaffey, Eamonn ................................................................................... P83

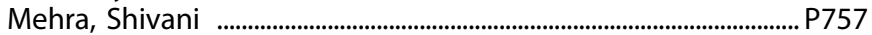

Mehralizade, Aydan .......................................................................................... P566 
Mehta, Aarush ......................................................................................................... P4

Mehta, C. Christina ............................................................................... P23

Mehta, Namrataa ....................................................................................... P64

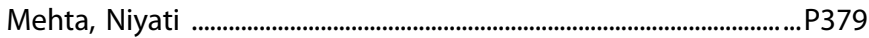

Meigs, Jennifer ……………………………………….............................. P403

Meija, Amanda ............................................................................................ P784

Meijer, Onno ........................................................................................P318

Meinel, Michael ...................................................................................... P582

Melas, Philippe ....................................................................................... P759

Melchior, James ............................................................................................... P86

Melendez, Carlos ......................................................................................... P687

Meller, Jarek ..........................................................................................P550

Mellick, William ................................................................................................... P307

Mellios, Nikolaos .......................................................................................... P634

Meltzer, Herbert .......................................................................................... P480

Meltzer-Brody, Samantha ……………………………………........ 53, P351

Melugin, Patrick ……………………................................................ P655

Melzer, Sarah ..................................................................................P331

Menard, Caroline ............................................................................... 4.2, P254

Mendes-Silva, Ana Paula ............................................2248, P344, P354

Mendez, Emily ................................................................................................... P688

Mendez, Ian ..........................................................................................P706

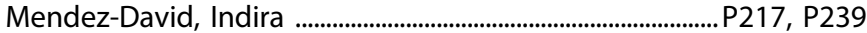

Mendlewicz, Julien .................................................................................. P240

Mendoza, Mariel ......................................................................................P664

Menegatti, Ricardo ..................................................................................... P407

Merad, Miriam ...................................................................................... P352

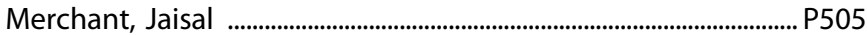

Merchenthaler, Istvan ................................................................................... P327

Meredith, Lindsay ..................................................................................... P734

Merikangas, Kathleen ......................................................................P121

Merk, Taylor ...................................................................................................P163

Merranko, John ................................................................................ P363

Merritt, Christina _..................................................................... P412, P636

Mertens, Lea ...................................................................................................P350

Meruelo, Alejandro .................................................................................P763

Mervis, Carolyn ..................................................................................... P147

Mesholam-Gately, Raquelle I. ..............................................................P487

Messinger, John .............................................................................. P588

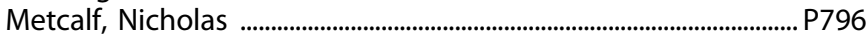

Metzler, Thomas ……………………………………………………... P42

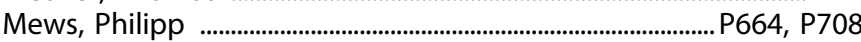

Meyer, Collin .............................................................................................P324

Meyer, Heidi .................................................................................................. P30

Meyer, Jeffrey ...............................................................................P425, P767

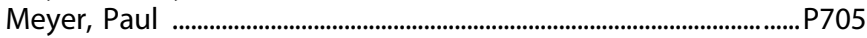

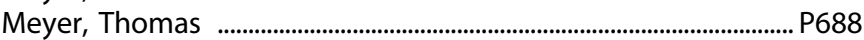

Meyer, Timothy ........................................................................................ P510

Meyerink, Michael ..................................................................................... P67

Meyer-Lindenberg, Andreas ..................................................................37.4

Meyers, Jacquelyn ................................................................................ P184

Meyers, Robert ............................................................................................. P5 P56

Michaelides, Mike ...............................................................175, P233, P729

Michel, Christina ................................................................... P448

Michopoulos, Vasiliki ........................................ P18, P48, P53, P64, P65, $P 70, P 72, P 170, P 317$

Miczek, Klaus P635

Migias, Yianni .........................................................................................P739

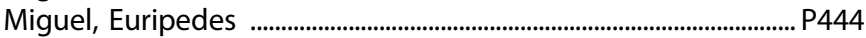

Mihali, Andra ................................................................................................... P473

Mikheev, Artem ........................................................................................ P19

Miklowitz, David ....................................................................................... P341

Mikofsky, Rachel ................................................................................... P459

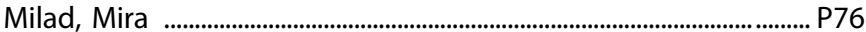

Milad, Mohammed ........................................................................................... P76, P92

Milanese, Marco .......................................................................................... P87

Milanovic, Snezana ......................................................................................... P5

Miles, Amy ................................................................................................... P1 139
Milham, Michael

$\mathrm{P} 121, \mathrm{P} 137, \mathrm{P} 822$

Miller, Alexander

P516

Miller, Andrew

P310, P498

Miller, Daniella ............................................................................................. P244

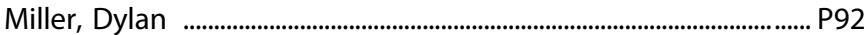

Millett, Caitlin ...............................................................................................P298

Mills, Fergil ................................................................................................... P27

Mimura, Masaru …................................................................. P390, P579

Minassian, Arpi ................................................................................................ P815

Mingardi, Jessica ....................................................................................... P87

Minhajuddin, Abu ........................................................ P1, P20, P241, P394

Minier-Toribio, Angelica .............................................P221, P342, P657

Minnig, Margaret ................................................................................. P649

Mintz, Emily ................................................................................................P133

Miracle, Sophia _............................................................................................ P649

Miranda, Alannah ...................................................................... P303, P815

Miranda, Raymundo .............................................................................. P672

Miranda, Jr., Robert ....................................................................P639, P734

Mirmina, Julianne ................................................................................... P313

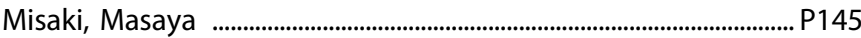

Mischoulon, David .........................................................................................P361

Mishra, Himanshu ................................................................................. P401

Mishra, Jyoti .................................................................................................... P83

Mishra, Ram ...........................................................................................P127

Misra, Madhusmita ................................................................................P186

Missig, Galen ................................................................ P35, P278, P379

Mitchell, Colter ............................................................................................ P140

Mitchell, Jennifer ........................................................................................ 15

Mitra, Swarup ...............................................................................................P612

Mitsi, Vassiliki ....................................................................................P730

Miyakawa, Tsuyoshi .................................................................................... P508

Miyakoshi, Makoto ........................................................................................ P552

Miyaoka, Tatsunori ............................................................................... P553

Mizrahi, Romina ........................................................................................ 13.3

Mizuno, Akiko …………………………………………............................. P11

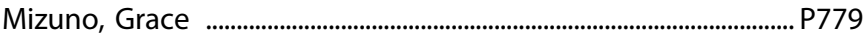

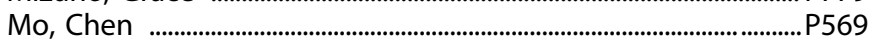

Moaddel, Ruin .............................................................................................. P233

Moazen-Zadeh, Ehsan ......................................................................P742

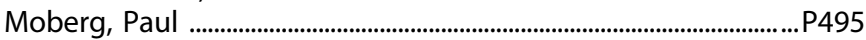

Modabbernia, Amirhossein ..............................................................P162

Moeller, F. Gerard ....................................................................7, P583, P693

Moghaddam, Bita ................................................ 22, 22.3, 40, P90, P702

Molenaar, Nina ............................................................................................ P178

Molina, Henrik ............................................................................................ P744

Molina, Juan ..............................................P25, P476, P573, P576, P827

Moline, Margaret .......................................................................................... P585

Molla, Hanna ................................................................................ P682, P818

Mollon, Josephine .............................................................................P512

Momenan, Reza ...............P285, P648, P678, P680, P692, P700, P726

Mondal, Abhijit .....................................................................................P512

Monk, Catherine ......................................................................................... P149

Monk, Christopher S. ........................................................................... P140

Monk, Kelly ......................................................................................................P363

Monosov, llya ...............................................................................................35.2

Montalvo-Ortiz, Janitza .......................................................... P61, P736

Montani, Caterina ……………………………………………………......... P570

Montemitro, Chiara ................................................................................ P670

Montgomery, Sarah ............................................................................... P177

Montgomery, Stuart .................................................................................. P240

Monti, Elisa .................................................................................................... P98

Montoya, Amanda ..................................................................................... P734

Monty, Gillian ................................................................................................. P99

Moody, David ...........................................................................................P694

Moody, Teena ................................................................................................P4

Moon, Chan Hong ................................................................................... P284

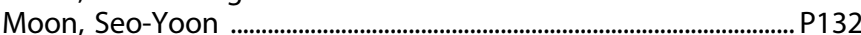

Moon, Sung-Hyun .......................................................................................... P500 
Moore, Andrew ..................................................................................... P610

Moore, Catherine ................................................................................... P651

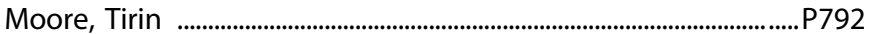

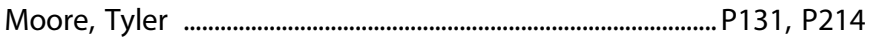

Morales, Austin ..................................................................................... P111

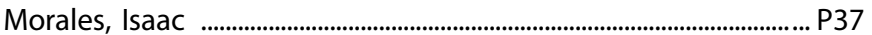

Morales, Kimberly ....................................................................................... P15

Morales, Marisela ...................................................................................... P52

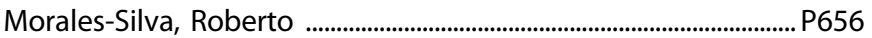

Moran, Landhing ....................................................................................P728

Moran, Lauren ..............................................................P475, P481, P505

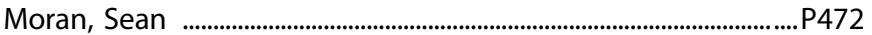

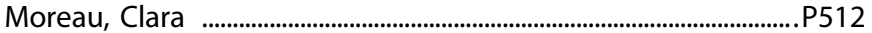

Morel, Carole .....................................................................................P177

Moreland, Angela .................................................................................P790

Morfini, Francesca ..................................................................................P467

Morgado, Pedro …………………...................................................... P440

Morgan, Erin .........................................................................................P815

Morgan, Janiece D. ..................................................................................... P716

Morgan, Judith ..................................................................................................26.2

Morici, Juan Facundo .................................................................................... P802

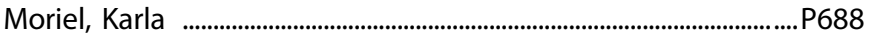

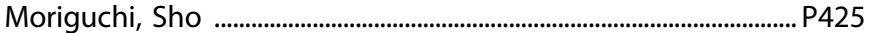

Morilak, David .................................................................................. P66

Morishita, Hirofumi ..................................................................................P808

Morisseau, Christophe ..................................................................................P189

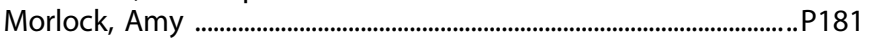

Morlock, Robert ................................................................................... P181

Morningstar, Mitchell ................................................................................... P604

Morris, Allison ..............................................................................................P629

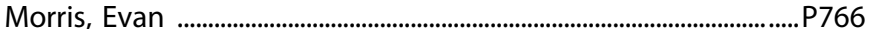

Morris, James ……………………………..............P678, P680, P687, P700

Morris, Laurel .........................................................................................P230

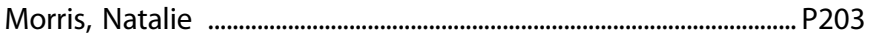

Morris, Patrick J. ....................................................................................... P233

Morris, Stewart ............................................................................................P778

Morrison, Kathleen ................................................................................P113

Morrison, Randall ................................................................................... P377

Morrow, A. Leslie ....................................................................... 31, 31.2

Morrow, Jonathan ................................................................................. P626

Moser, Amelia ………………………........................................................P341

Moser, Hannah ........................................................................................ P483

Moses, Rebecca L. .........................................................................P539

Moskal, Joseph ………………………………………………........ P311

Mostofsky, Stewart ....................................................................................... P784

Motamedi, Massoud ......................................................................................... P412

Motelow, Joshua ........................................................................................... P566

Mou, Ta-Chung M. ................................................................................. P327

Moxon-Emre, Iska .............................................................. P418, P469

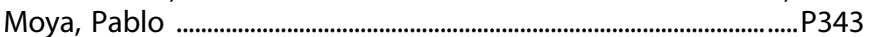

Moyett, Ashley ............................................................................P455, P506

Muckerman, Julie ........................................................................................ P126

Mueller, Bryon ..............................................................P564, P605, P817

Mueller, Devin ..................................................................................... P656

Mueller, Martina ......................................................................................... P406

Mueser, Kim ...........................................................................................P556

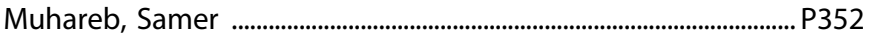

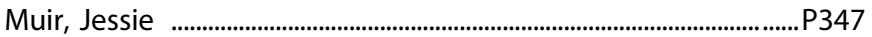

Mujica, Christin ..........................................................................................P505

Mukerji, Shibani ......................................................................................... P4 11

Mukhin, Alexey G. ................................................................................P716

Muller Ewald, Victoria ..............................................................................P321

Mulsant, Benoit ...............................................P17, P231, P314, P354, P497

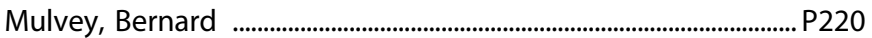

Muñoz Zamora, Andrea ........................................................................... P60

Muralidhar, Sumitra ................................................................................ P477

Muro, Karina

Murphy, Kathleen ............................................................................... P809

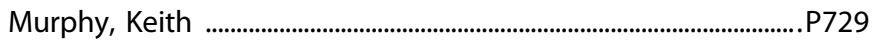

Murphy, Michael ...................................................................................... P522

Murphy, Nicholas ................................................................................ P328

Murphy, Ryan ...............................................................................................P563

Murphy, Susan ........................................................................................ P345

Murphy-Weinberg, Virgina ..................................................................... 336

Murray, Conor ................................................................................................. P682

Murray, Laura .................................................................................P769

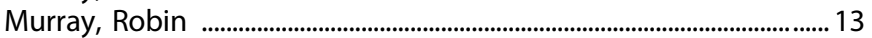

Murray, Susan .........................................................................................P116

Murrough, James .................................................... P68, P230, P352, P370

Murtagh, Lorainne .................................................................................... P154

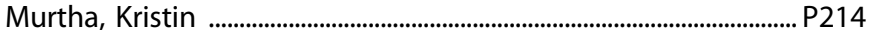

Murthy, Venkatesha ...............................................P462, P514, P560

Murty, Vishnu ………...................................................................... P71, P788

Musazzi, Laura ........................................................................................ P87

Muscat, Stephanie ........................................................................................ P16

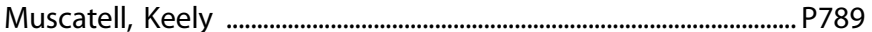

Muvvala, Srinivas ...................................................................................... P6 639

Muzekari, Benjamin ...................................................................................P122

Mwangi, Benson .............................................................................................. P803

Mychaskiw, Marko .................................................................................. P541

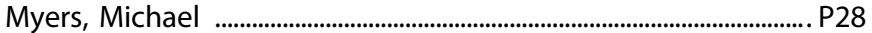

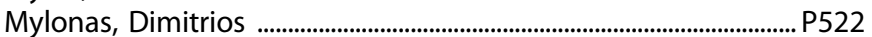

Myoraku, Alison ......................................................................... P22, P312

Myroshnychenko, Maxym ...........................................................P459

Nabulsi, Nabeel ...............................................................P210, P592, P766

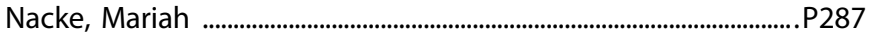

Nadig, Ajay ...................................................................................................... 124

Nadkarni, Girish ........................................................................................... P62

Nagamatsu, Sheila ................................................................ P61, P736

Nagel, Bonnie .............................................................................................P763

Najafi, Bijan ..................................................................................................P127

Najera, Katherine ...................................................................................... P68

Nakagawa, Shinsaku ................................................................................. P553

Nakajima, Shinichiro ...........................................................................P579

Namba, Mark ........................................................................................P770

Nambiar, Ankita ……………………....................................................... P187

Namburi, Praneeth ........................................................................................... P267

Nandy, Indrani ......................................................................................... P2 P

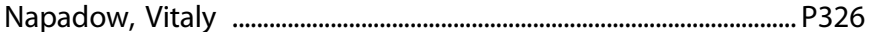

Narla, Vishal ...........................................................................................P380

Narr, Katherine ........................................................................................... P515

Nasca, Carla ...................................................................................P244, P254

Nash, Tiffany ............................................................................... P107, P147

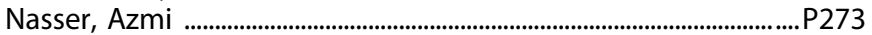

Natesan, Sridhar ......................................................................................... P4 P

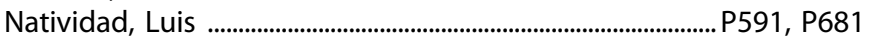

Navarro Gomez, Maria J. ................................................................ P720

Navickas, Albertas .................................................................................. P744

Nawabudike, ljeoma ..............................................................................P171

Nawaz, Uzma ................................................................................................P475

Nawreen, Nawshaba ............................................................................P300

Nebel, Mary Beth ................................................................................P784

Needham, Patrick ................................................................................................. P5

Neelakantan, Uma ...............................................................................P171

Neigh, Gretchen .................................................................... P23, P170

Neilson, Chiara ..................................................................................... P341

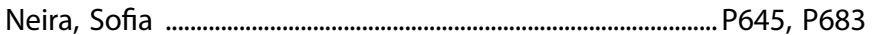

Neisewander, Janet ............................................................................P634, P770

Nelson, Brady ............................................................................................. P71

Nelson, Katherine ...................................................................................... P641

Nelson, Nellie ..................................................................................................P108

Nelson, Tyler ..................................................................................P704

Nemeroff, Charles ................................................................................ 21, P793

Nennig, Sadie ............................................................................................P263

Nestler, Eric

P177, P221, P254, P301,

P342, P657, P708, P730, P744 
Nestsiarovich, Anastasiya P303

Neta, Maital

Neuhofer, Daniela

P615, P717

Neumaier, John

$\mathrm{P} 45, \mathrm{P} 644$

Nevado, Jose

P123

New, Antonia .......................................................................................P210

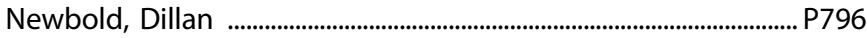

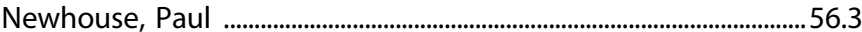

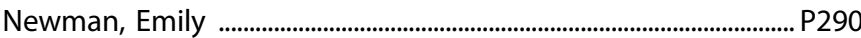

Neylan, Thomas ........................................................................................... P42

Nguyen, Claudia V. ..................................................................................... P559

Nguyen, Jacqueline .................................................................................P127

Nguyen, Jacques ................................................................................. P589

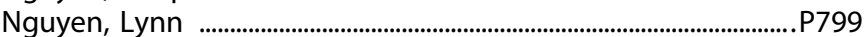

Nguyen, Nghi ...........................................................................................P308

Nguyen, Nhien ............................................................................................. P189

Nguyen, Tammy ..................................................................................... P337

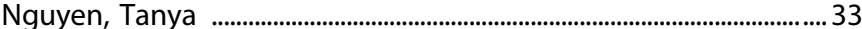

Nguyen, Thanh ............................................................................ H. P331

Ngyuyen, Vien ............................................................................................ P327

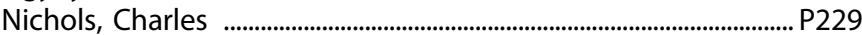

Nicholson, Andrew ...................................................................................43.1

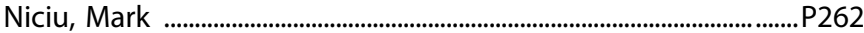

Nickl-Jockschat, Thomas .......................................................................... P494

Nicol, Ginger ................................................................................... P507, P796

Niedringhaus, Mark ............................................................................P724

Nielsen, Kathryn ................................................................................P505

Nielsen, Mette .................................................................................. P542

Niendam, Tara ................................................................................ P457, P555

Nierenberg, Andrew .....................................................P288, P298, P361

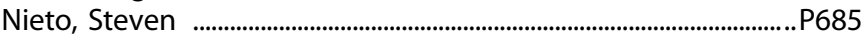

Nievergelt, Caroline ........................................................................................ P38

Nikolitch, Katerina ...............................................................................P367

Nikolova, Yuliya ...................................................................................... P9, P139

Nikzad, Amir ...................................................................................................P496

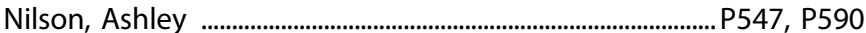

Nimarko, Akua .......................................................................................... P393

Nimgaonkar, Vishwajit ............................................................................. P363

Ning, Courtney ................................................................................. P498

Nishioka, Tadaaki .............................................................................P823

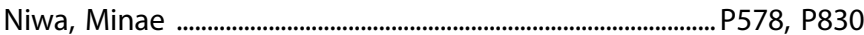

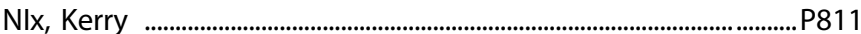

Nixon, Sara ..................................................................................................P610

Niznikiewicz, Margaret ...........................................................P467, P545

Noda, Yoshihiro ................................................................................... P579

Nolan, John ......................................................................................................P248

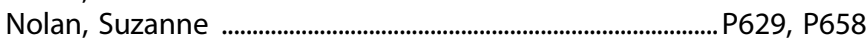

Norcia, Anthony ..................................................................................... P811

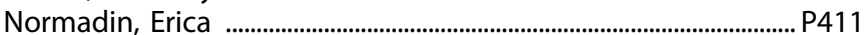

Normandin, Marc ................................................................................. P500

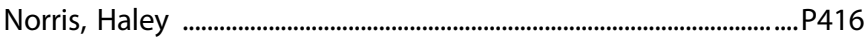

Northoff, Georg ............................................................................................ P467

Notarangelo, Francesca M. ..............................................................P569

Novotny, Stephanie .............................................................................P172

Nucifora, Frederick ...................................................................................... P449

Nucifora, Leslie ....................................................................................... P449

Nuechterlein, Keith ..................................................................... P499, P515

Nunes Marchette, Renata .............................................................P731

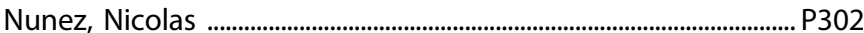

Núñez-Ríos, Diana ..................................................................................... P61

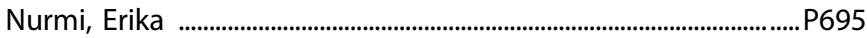

Nurnberger, John ................................................................................... P28

Nutt, David ..................................................................................................P800

Nutinen, Makiah ...................................................................................P310

Nuzhny, Nicholas ............................................................................................. P32

Nwulia, Evaristus ...................................................................................... P773

Nye, Jonathon ………………………………………………….... P317

Nye, Kathleen ..............................................................................................P555

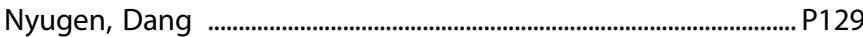

O'Connor, Alexandra ............................................................................. P331

O'Donnell, Patricio ...................................................................................... P462

Oathes, Desmond ......................................................................... P323, P392

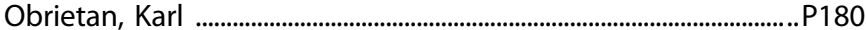

Obrig, Hellmuth .............................................................................................. P141

Ochiai, Yukiko ..............................................................................................P426

O'Connell, Emma ............................................................................................. P778

O'Connor, Meaghan .......................................................................................... P8

O'Connor, Richard ..................................................................................... P194

O'Connor, Shayna .......................................................................................... P764

$\varnothing$ degaard Nielsen, Mette ......................................................................P526

O'Donnell, Carly .................................................................................................. P148

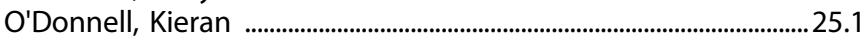

O'Donnell, Lauren ........................................................................................ P565

O'Donnell, Patricio ...................................................................................... 7, P514

O'Donovan, Aoife ....................................................................................... P42

O'Donovan, Sinead ............................................................................. P322

Ofotokun, Igho .......................................................................... P23, P170

Ogawa, Sachie ......................................................................................... P528

Ogirala, Ajay …………………………………………………......P491, P517

O'Gorman, Cedric .......................................................................P245, P246

Ojeda-Torres, Giovanne .......................................................................P365

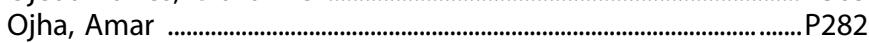

Okiyama, Ryoichi .................................................................................... P426

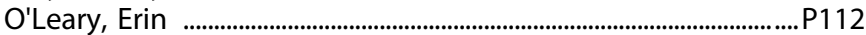

O'Leary, Timothy .......................................................................................... P477

Oler, Jonathan A. ................................................................................. P78

Olfson, Emily ...................................................................................................... P146

Olié, Emilie ...................................................................................................P296

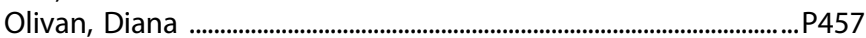

Olive, M. Foster .......................................................................................... P770

Oliveira, Amanda .............................................................................. P444

Oliver, Lindsay ..................................................................................P418, P469

Olivetti, Pedro ………………………...........................................................P534

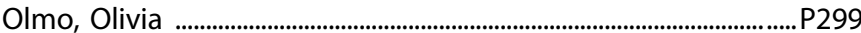

Olsen, Elizabeth ......................................................................................... P2 206

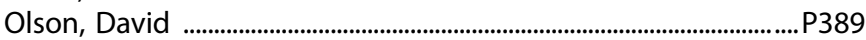

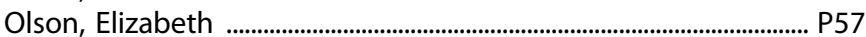

Olson, Jacob ...............................................................................................P267

O'Malley, Stephanie ...............................................................................P639

Ombach, Hendrik J. ................................................................................... P338

Ongur, Dost ............................................... P474, P481, P505, P556, P565

Ophoff, Roel ..............................................................................................P499

Oquendo, Maria $\ldots \ldots \ldots \ldots \ldots \ldots \ldots \ldots \ldots \ldots \ldots$

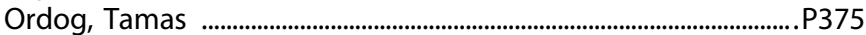

Ordones Sanchez, Evelyn ......................................................................... P684

Orsini, Caitlin ............................................................................... 22.1, P640

Orso, Rodrigo .......................................................................................P134

Ortinski, Pavel ...........................................................................................P751

Ortiz-Juza, Maria .......................................................................................... P39

Osanai, Hisayuki .............................................................................................. P528

Osman, Aya .............................................................................................P715

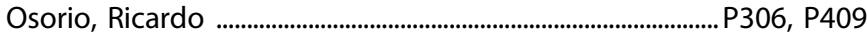

Ostrand, Caroline ............................................................................. P31, P57

Oswell, Corinna ....................................................................................... P600

Othy, Shivashankar ..................................................................................... P1 14

Otis, James .................................................................................... 45.3, P713

Otten, Joy .................................................................................................. P96

Overbey, Jessica ............................................................................................... P68

Owen, James .........................................................................................P273

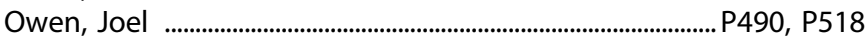

Owens, W Anthony ......................................................................................... P671

Oxenkrug, Gregory ................................................................................... P5

Ozburn, Angela ................................................................................ $34,34.1$

Ozerdem, Aysegul ................................................................................ P382

Ozger, Can .................................................................................................P144

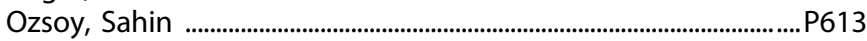


Paans, Nadine

Pabon, Elisa

Pace, Thaddeus

Pachas, Gladys

Pacheco, Jenni

Pacheco-Colón, lleana

Padilla, Viviana

Padilla-Coreano, Nancy

Padovani, Andrew

Padovano, Hayley Treloar

Pagani, Marco .

Pagliaccio, David

Paim Diaz, Alexandre

Pain, Scott

Pal, Shibangi

Palka, Jayme

Pallanti, Stefano

Palmer, Abraham

Pamidighantam, Preetha

Pan, Jen

Pan, Jullie

Pan, Tao

Panchal, Priyanka

Pandey, Ashwini

Pandey, Gayathri

Pandey, Subhash

Pandina, Gahan

Panlilio, Leigh

Pantazopoulos, Harry

Paoli, Caterina

Papademetris, Xenophon

Papadopoulos, Elektra

Papaleo, Francesco

Papanikolopoulos, Nikos

Papotto, Claudio

Papp, Mariusz

Pappagallo, Marco

Paredes, Denisse

Pareek, Pallavi

Parekh, Ankit

Parekh, Puja

Parent, Justin

Parent, Sophie

Paret, Christian

Parida, Kurren

Parikh, Sagar

Parikh, Vinay

Parise, Eric

Parise, Lyonna

Park, Catherine

Park, Royce

Park, Seojin

Parker, Jones

Parker, Krystal

Parrino, Liborio

Parsey, Ramin

Parvaz, Muhammad

Pas, Pascal

Pascoal, Tharick

Pasqualetti, Massimo

Passarell, Julie

Pasternak, Ofer

Patarino, Makenzie ........................................................................................ P672

Patel, Gaurav

Patel, Nishi

Patel, Reesha

Patel, Sachin
P17

P682

P48, P53, P72

$P 606, P 631$

... 37

P587

P555

$39,39.3$, P267

P555

P639

P137, P570

P325, P822

P803

P581

P815

P406

P440

P677, P695, P701, P703, P705, P746

P436

P472, P522

.P284

P744

P364

P184

P184

P234, P725, P747

5, P288

.P661

P236

P87

P171

.... 36

P570

P421

P642

P320

P315

P66

P787

P409

P213

P146

P153

P447

P687

P371

P26

P221，P249, P301

P249, P301, P352

P127

P461

P541

... P89

P321

P581

P391, P404

P164

. P79

P10

P259, P570

P490

P487

P607

P527

. P26

P27, P672, P681

....... P40, P86, P299, P743
Pathak, Jyotishman P62

Pati, Dipanwita

P417

Patino, L. Rodrigo

P397, P398, P399

Patra, Braja

Patrick, Bailey

... P62

Patrick, Finan .................................................................................................P353

Patrick, Regan .............................................................................................. P17

Patterson, Beth ..................................................................................... P440

Patterson-Cross, Joanna ........................................................................P123

Pattie, Elizabeth A. ................................................................................P539

Patton, Michael S. ............................................................................. P327

Patwell, Ryan ........................................................................................P725

Paul, Elisabeth ....................................................................................... P771

Paul, Feuerstadt ....................................................................................... P334

Paul, Stephen ............................................................................................P510

Paula, Dayana ................................................................................................P587

Paulus, Martin ........................................................12, 33, P55, P145, P209,

P242, P287, P652, P719

Pazdrak, Konrad

P412

Pearlson, Godfrey

P487, P572, P669

Pechtel, Pia

P389

Peck, Emily

..P740

Pedapati, Ernest

$\mathrm{P} 173, \mathrm{P} 428$

Pedersen, Nancy ....................................................................................... P445

Pedrino, Gustavo Rodrigues ..............................................................P407

Pekar, James ....................................................................................................P353

Pekarskaya, Elizabeth ................................................................................ P292

Pellegrini, Luca ....................................................................................P434

Peltz, Gary

$\mathrm{P} 694, \mathrm{P} 735$

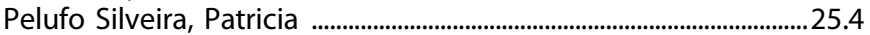

Pena, Catherine ………………….................................... 29.4, P254, P730

Peng, Christine ............................................................................................. P8 825

Peng, Xiaolong ....................................................................................P201

Pennington, Zachary ...................................................................... P84, P744

Penninx, Brenda .......................................................................P218, P318

Pereira, Carlos Alberto .........................................................................P444

Pereira, Sandra ......................................................................... P509, P559

Perez, Stephanie ............................................................................................ P6

Perez de la Cruz, Veronica ...................................................................... 6.2

Perez-Edgar, Koraly ....................................................................................26.1

Perez-Perez, Yobet ...............................................................................P656

Perez-Rodriguez, M. Mercedes ............................... 32, P98, P178, P210

Perez-Torres, Joshua ...........................................................................P656

Perino, Michael ................................................................................................ P28

Perkins, Diana ......................................................................... 19, 52, P545

Perlstein, Samantha ....................................................................................P799

Perreault, Melissa ...................................................................................... P356

Perrone-Bizzozero, Nora ....................................................................... P634

Perry, Pamela ................................................................................................P493

Peskind, Elaine .......................................................................................... P777

Peters, Craig ............................................................................................. P59

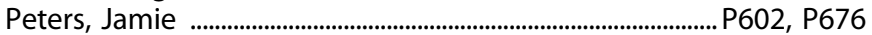

Peters, Mette .............................................................................................P461

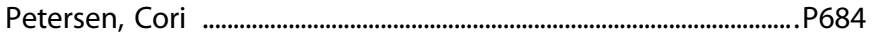

Peters-Lawrence, Marlene ..................................................................P429

Peterson, Bradley ..................................................................................... P149

Peterson, Elena ............................................................................................. P341

Peterson, Roseann .................................................................................. P226

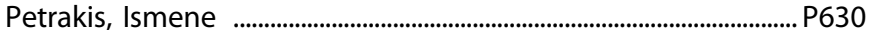

Petrella, Michele ........................................................................... P36, P711

Petrides, Georgios ...................................................................P406, P810

Petterway, Felicia ..................................................................................... P186

Petty, Gordon .......................................................................................................P269

Peyton, Lee .........................................................................................P662

Phadke, Rhushikesh .................................................................................... P3 31

Pham, Aidan L. ......................................................................................... P387

Pham, Diana

P704

Phan, K. Luan

.. P185, P260 
Phelps, Elizabeth

Philip, Noah

Philip, Vivek

Philliips, Robert

Phillips, Hannah

Phillips, Jennifer

Phillips, Karran

Phillips, Mary

Phillips, Megan N.

Phillips, Rachel

Phillips-Jones, Taylor

Piacentino, Daria

Piantadosi, Anne L.

Piantadosi, Sean

Picone, Joseph

Pietrzak, Robert

Pihl, Robert

Piiomelli, Daniele

Piiwaa, Kayla

Pillai, Anilkumar

Pina, Melanie

Pine, Daniel

Pines, Adam

Pinna, Graziano

Pinto Tomás, Adrián

Pires, Andrea

Pires-dos-Santos, Isabela

Pisano, Ilaria

Pisoni, Angela

Pitts, Elizabeth

Pizzagalli, Diego

Plawecki, Martin H. ..

Plaza-Jennings, Amara

Pletnikov, Mikhail

Plitman, Eric

Plotkin, Joshua

Pocivavsek, Ana

Pocuca, Nina

Poffenberger, Chelsie

Poh, Jia-Hou

Pöhlmann, Max

Pokhvisneva, Irina

Poldrack Russel ……………………………...................P146

Polesskaya, Oksana .................................................................................. P705

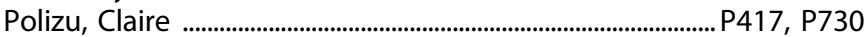

Pollock, Bruce ...................................................................................................P314

Pomara, Nunzio ........................................................................................P306

Pong, Sovannarath ................................................................................ P504

Ponomareva, Olga ........................................................... P95, P611, P698

Poore, Holly .........................................................................................P703

Pope, Harrison ........................................................................................... P183

Popiolek, Michael .................................................................................. P510

Popoli, Maurizio ......................................................................................... P87

Porjesz, Bernice ...................................................................................................... P184

Porkka, Fiona ..........................................................................................P596

Portanova, Jake …………………………………………………………..... P4 465

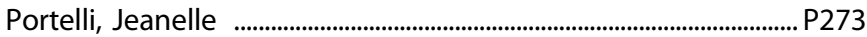

Porteous, David ..................................................................................... P778

Porter, Christopher J.H. ............................................................................. P4

Posani, Lorenzo ........................................................................................... P537

Posner, Jonathan ..........................................................P149, P196, P299

Potenza, Marc ............................................................................... P749, P804

Potter, Alexandra ..................................................................................... P169

Potter, Julia ..................................................................................................P228

Potter, Kevin ..................................................................................P606, P631

Potter, Liam E. ................................................................................................... P327

Potter, William $\ldots \ldots \ldots \ldots \ldots \ldots \ldots \ldots \ldots \ldots \ldots \ldots \ldots$
Považan, Michal .............................................................................................. P353

Poweleit, Ethan ..................................................................................P159

Powell, Gregory ………………............................................................ P634

Powell, Reid T. ............................................................................................ P308

Powers, Abigail ..........................................P18, P48, P53, P65, P72, P79

Pozsonyivoa, Sofia ....................................................................................... P762

Pradhan, Sameer ........................................................................................... P496

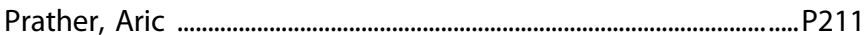

Prensky, Zack .........................................................................................P507

Preston, Kenzie ..................................................................................... P728

Prettyman, Greer ………………………………………………………....... P571

Prevot, Thomas .......................................................................................... P344

Price, Lawrence ...................................................................................... P250

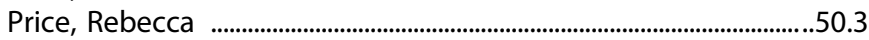

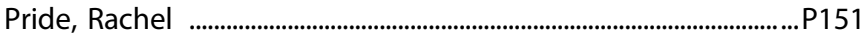

Prieto, Miguel .....................................................................................................

Printz, David ............................................................................................ P83

Prisciandaro, James ....................................................................... P307

Prisinzano, Tom ............................................................................................ P782

Prochaska, Trinidi ...................................................................................P168

Prokai, Laszlo ........................................................................................P327

Prokai-Tatrai, Katalin .................................................................................. P327

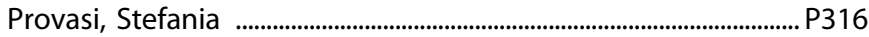

Prudic, Joan ..................................................................................................P406

Pruitt, April .................................................................................................. 171

Pruitt, Christopher ....................................................................................... P29

Pryce, Kerri ..........................................................................174, P419, P730

Pryor, Alison ..............................................................................................P421

Przygodzki, Ronald ............................................................................................. P4

Pu, Yaoyu .....................................................................................................P405

Puig, Stephanie ................................................................................................. P765

Pulley, Desta ........................................................................................................P607

Pultorak, Katherine J. ........................................................................... P327

Pung, Merideth A. ................................................................................. P29

Purcell, Shaun ..........................................................................................P522

Pyarajan, Saiju ...................................................................................... P477

Pyter, Leah ........................................................................................................P180

Qadir, Houman ......................................................................................... P814

Qang, Guogiang ..................................................................................P522

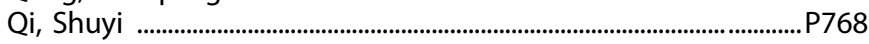

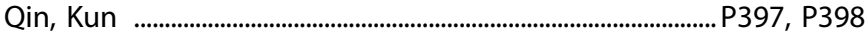

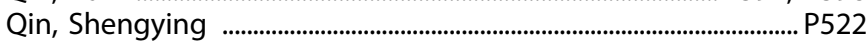

Qirici, Garry ..............................................................................................P415

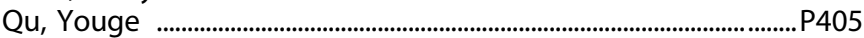

Quave, Cana ...............................................................................................P745

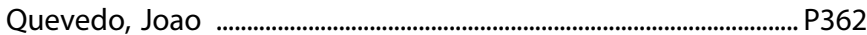

Quevedo, Karina .............................................................................................4. 43.3

Quian, Jessica ......................................................................................P479

Quijano Cardé, Natalia .............................................................................. P686

Qvist, Johanna ........................................................................................... P759

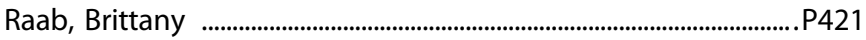

Rabinak, Christine ....................................................................................... P59

Rabinowitz, Alon ........................................................................................ P100

Rabinowitz, Jonathan .......................................................................... P100

Radar, Anna ........................................................................................................... P464

Radoman, Milena ............................................................................................ P2 260

Ragland, J. Daniel ................................................................................. P457

Rahat, Maryam ........................................................................................... P440

Rahman, Rayees ....................................................................................... P120

Rahman, Samir ............................................................................................ P461

Rai, Narayan .............................................................................................P773

Raines, Shane ..........................................................................................P2 235

Rainville, Jennifer ........................................................................................... P370

Raio, Candace .............................................................................................P826

Rais, Rana ...................................................................................................P590

Rajagopal, Lakshmi ............................................................................. P480

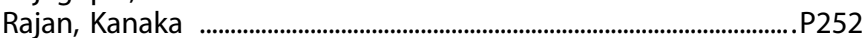

Rajeevan, Nallakkandi .................................................................................... P477 
Rajji, Tarek

Rakela, Samantha

P248, P314, P497

Ralevski, Elizabeth

..P721

Ramaekers, Johannes

Ramakrishnan, Aarthi

Ramakrishnan, Nithya

Ramamurthy, Chethan

Raman, Peter

Ramanathan, Dhakshin

Ramchandani, Vijay

Ramey, Tanya

Ramirez-Sanchez, Leonardo

Ramirez-Zamudio, Andres

Ramos, Jeremiah

Ramos, Paula

Ramos-Cejudo, Jaime

Ramsay, lan

Ramsey, Amy

Ramsey, Cheldyn

Ramsey, Laura

Rana, Nilanjana

Rangarajan, Anusha

Rankin, Ingrid

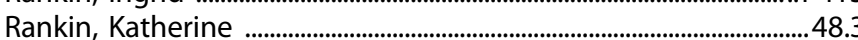

Ransom, Janet ..................................................................................................... P639

Rao, Vinod

Rapalino, Otto

Rapaport, Mark

Rapoport, David

Rapp, Amy

Rasenick, Mark

Rasgon, Natalie

Raskin, Marissa

Raskind, Murray

Rasmussen, Jerod M.

Rasmussen, Kurt

Rasmussen, Steve

Rassnick, Stephanie

Rathi, Yogesh

Ratti, Emiliangelo

Rauch, Scott

Raus, Anthony

Ravi, Meghna

Ravina, Bernard

Rawls, Eric

Ray, Lara

P824

P328

P12

P793

57, P83

P678, P680, P686, P687, P700

7, 7.11, P583, P693

... P73

P178

P192

..P444

P306

...P511, P564

P431

P242

P159

P271

P825

P405

3, P361, P369

P409

P433

P369

P22, P312, P369

..P54

P99, P777

P106

.20

35.4

P288

P487, P565

P560

... 31

P108

P65

P235

..P150, P595

Ray, Reese Pat

Raznahan, Armin

Reasons, Sarah

Rebecca, Betensky

Reda, Mariam

Redwine, Laura S.

Reed, Hannah

Regnier, Grace

Reichel, Carmela

Reichenberg, Abraham

Reichert Plaska, Chelsea

Reid, Meredith

Reigle, James

Reigle, Jim .

Reimer, Adriano

Reiner, Benjamin

Reissner, Kathryn

Rej, Soham

Remington, Gary

Remoaldo Trambaiolli, Lucas

Ren, Boyu

Rendina, Danielle
Rene, Alexandra P760

Renger, John

P493, P500

Renshaw, Perry

Renteria, Miguel

Rentsch, Christopher

P237, P281, P338

Rentschler, Katherine ........................................................................... P557

Research Group, Traumatic Stress Brain ........................................... P61

Research Team, 23andMe

Resendez, Alexis

P746

Ressler, Kerry

P89, P309

P53, P65, P70, P79,

P91, P93, P95, P195, P267, P290, P788

Rethman, Jacob ................................................................................... P550

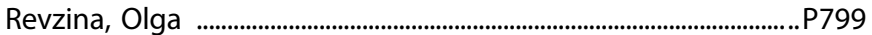

Reyes, Abner P708

Reynolds, Charles ........................................................... P231, P354

Reynolds, Erik ...........................................................................................P365

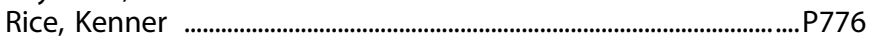

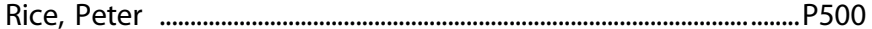

Richards, Anne ............................................................................................. P42

Richardson, Kimberlei ................................................................................P188

Richter, Kneginja ............................................................................................ P533

Ridout, Kathryn .................................................................................P211

Ridout, Samuel ....................................................................................... P211

Riedel, Marissa ........................................................................................... P78

Rihel, Jason ................................................................................................. P171

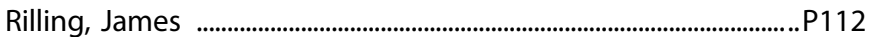

Rios, Lupita ............................................................................................P380

Risbrough, Victoria .........................................................................54, P38

Riskus, Sadie .............................................................................. P360, P370

Ritchie, Jobe L. .....................................................................................P768

Riva, Marco Andrea ....................................................................P316, P320

Roach, Brian

Roalf, David

P470, P503, P545, P561

Robakis, Thalia

Robbins, Trevor

P148, P214, P495

Roberto, Marisa

...P22

P800

Roberts, Amanda

P611

Roberts, Benjamin

P591, P611, P681

Roberts, Nicole

562, P576

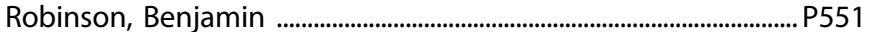

Robinson, Delbert

P506, P516

Robinson, Elise

$38.3, \mathrm{P} 124$

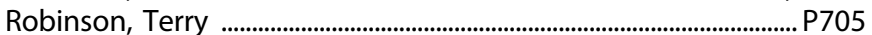

Rocchi, Federico .................................................................................... P570

Rocereta, Julia A. ................................................................................... P547

Rocks, Devin .............................................................................................. P80

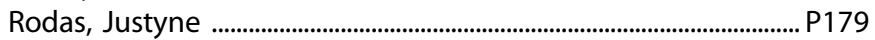

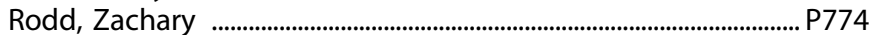

Rodriguez, Agustin ..................................................................................... P562

Rodriguez, Alyssa ........................................................................................ P3

Rodriguez, Carolyn .........................................................50, P440, P442

Rodriguez, Katrina M. .........................................................................P569

Rodriguez, Larry .................................................................................. P681

Rodriguez-Romaguera, Jose ................................................................... P39

Rodriguez-Torres, Genesis ...................................................................P656

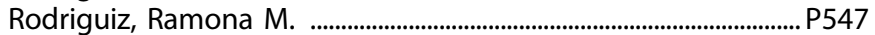

Roeckner, Alyssa ..................................................................................... P70

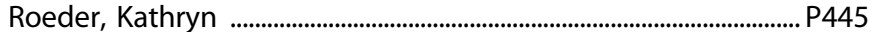

Roediger, Donovan .....................................................................P605, P817

Roepke, Troy .................................................................................... 24, 58

Roeske, Maxwell ........................................................................................... P486

Roffman, Joshua ...................................................................28, 28.4, P151

Rogers, Cynthia ……………………............................................ 59, P168

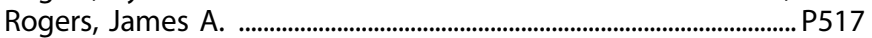

Rogers, Jeffrey ................................................................................P121, P667

Rog-Wilehlem, Natalie .....................................................................P793

Role, Lorna ........................................................................................ P4, P256 
Rollins, Oliver

Romanelli, Daniel

Rommel, Anna

P615

Romo Nava, Francisco

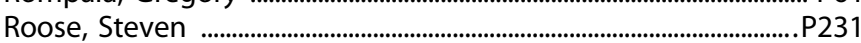

Ropchan, Jim ....................................................................................P592

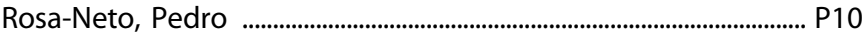

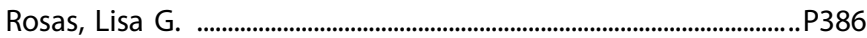

Rosas-Vidal, Luis ......................................................................................... P40

Rose, Jed E. .............................................................................................P716

Rose, Kristie ..............................................................................................P629

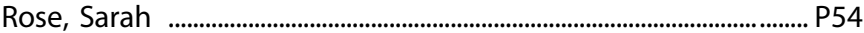

Roseboom, Patrick H. .............................................................................. P78

Rosell, Daniel ...........................................................................................P210

Roseman, Leor ................................................................................................ P800

Rosen, Howie ........................................................................................P791

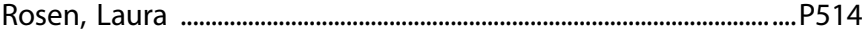

Rosenberg, Benjamin ..........................................................................P341

Rosenberg, Eric S. ................................................................................. P411

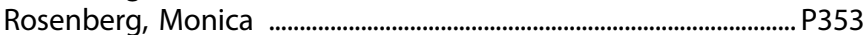

Rosenquist, Peter ......................................................................................... P406

Roshgadol, Jacob ………………..........................................................P213

Rosipal, Roman ...............................................................................P311

Rosoff, Daniel ...............................................................................................P778

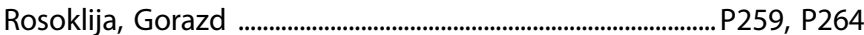

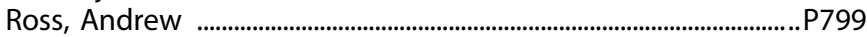

Ross, Christopher ................................................................................... P449

Ross, Jessica ......................................................................................................... P42

Ross, Rachel ...............................................................................................P195

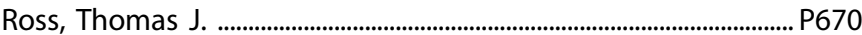

Rosso, Isabelle ................................................................................ P31, P57

Rostrup, Egill .......................................................................................P526

Rothbaum, Barbara ......................................................... P65, P70, P79

Rothschild, Anthony ............................................................................... P371

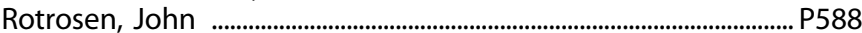

Rotstein, Natalie ...................................................................................... P439

Roussos, Panagiotis .................................................................................P575

Roussos, Panos ............................................................................ P461

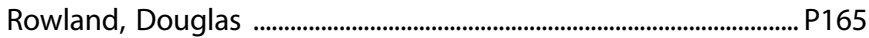

Rowley, Jordan ............................................................................................. P80

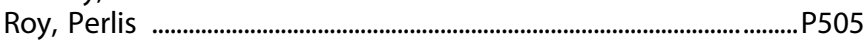

Roychoudhury, Rohon ....................................................................... P795

Rozovsky, Renata ............................................................................... P270

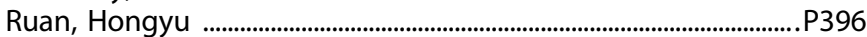

Rubin, Jonathan ..................................................................................... P273

Rubin, Sophie ........................................................................................P427

Rubinow, David .................................................................................... P351

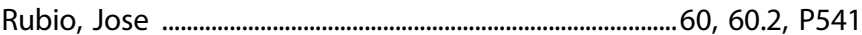

Ruck, Christian ....................................................................................... P445

Rudy, Melissa .........................................................................................P411

Rudzinskas, Sarah ................................................................................ P351

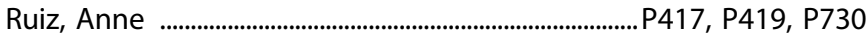

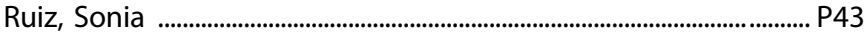

Rungby, Jørgen .............................................................................. P542

Runz, Heiko ......................................................................................P798

Ruparel, Kosha _............................................................................................... P495

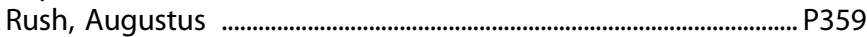

Rush-Goebel, Sage ............................................................................ P214

Rusinek, Henry ............................................................................................ P19

Russ, Jill .....................................................................................................P123

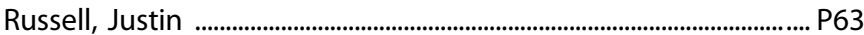

Russo, Scott .............. 47, P177, P230, P249, P254, P301, P342, P352

Ruttmann, Axel .............................................................................P533

Ryman, Sephira ................................................................................................. P374

Saad, Bassem ..............................................................................................P130

Saba, Natalie ..................................................................................P409

Sabatini, Bernardo ............................................................................................. P13
Sabb, Fred

P695

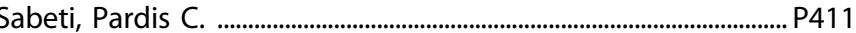

Sabino, Valentina ................................................................................... P649

Sacchet, Matthew ....................................................................................... P176

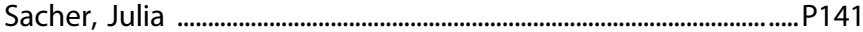

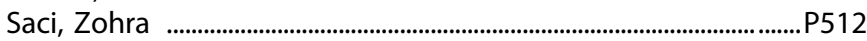

Sacson, Agostina ................................................................................... P802

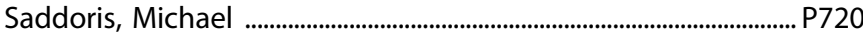

Sahani, Vyoma ...............................................................................P674, P807

Sahay, Amar .......................................................................................11, 11.4

Sailasuta, Napapon ....................................................................................... P579

Sajatovic, Martha ........................................................................................ P17

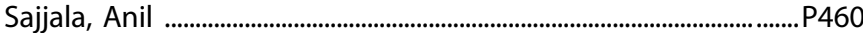

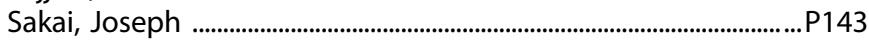

Sakamoto, Kotaro .................................................................................. P553

Sakolsky, Dara .............................................................................................. P363

Sala, Nathalie ........................................................................................ P87

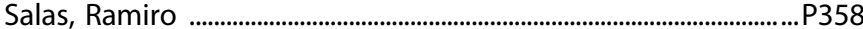

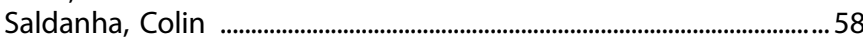

Salem, Nihal ...............................................................................................P611

Salery, Marine ..........................................................................................P301

Salimando, Gregory ................................................................................... P339

Salisbury, Dean ......................................................................................... P531

Salisbury, Ryan .................................................................................................. P7 765

Salmeron, Betty Jo ................................................................................. P670

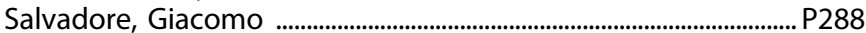

Salvatore, Jessica E. .................................................................................. P184

Samad, Muntaha ................................................................................................. P3

Sambo, Danielle ............................................................................................... P138

Samuels, Joshua .......................................................................................... P329

San Miguel, James …………………………………………………....... P757

Sanches, Marsal ............................................................................... 255, P803

Sanchez, Mar ..............................................................................................25.3

Sanchez, Raymond .................................................................................... P493

Sanchez, Victoria ........................................................................................ P460

Sanchez Soto, Marta .......................................................................... P233

Sanchez-Roige, Sandra .............................................................. P703, P746

Sanders, Bryson ....................................................................................... P739

Sanders, Christie ....................................................................................... P52

Sanders, Kevin .......................................................................................... P154

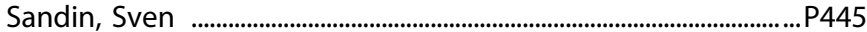

Sandler, Raizel ........................................................................................ P604

Sandman, Christina ............................................................................. P341

Sankar, Anjali .........................................................................................P364

Sano, Ellen .................................................................................................. P99

Santarriaga, Stephanie .......................................................................P504

Santiago, Adrienne ................................................................................. P259, P264

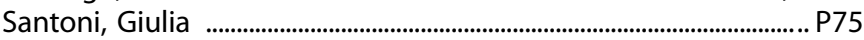

Santos, João Paulo Lima .......................................................................P304

Santos, Maria .................................................................................................P333

Saoud, Jay ...............................................................................................P546

Saperstein, Alice .................................................................................... P525

Sapienza, Frank .......................................................................................... P315

Saraiva, Erlandson .......................................................................................... P444

Sarna, Kaylee .............................................................................................. P17

Sarpal, Deepak ........................................................................... P538, P568

Sastre, David .........................................................................................P570

Sathe, Anish ...........................................................................................P815

Satterthwaite, Theodore .........................................47, P148, P214, P571

Saudagar, Vikram ....................................................................................... P269

Savage, Julie ...........................................................................................P114

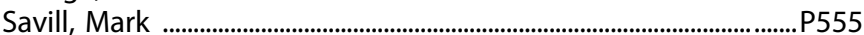

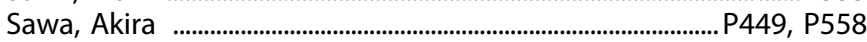

Saxe, Michael ............................................................................................ P56

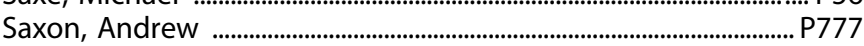

Sayrafizadeh, Negar ............................................................................... P179

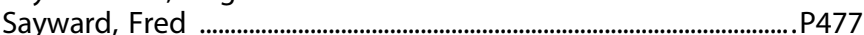

Scaini, Giselli ………………………..............................P255, P275, P362 
Scammell, Thomas .

Scarante, Franciele F.

P582

Schaadt, Gesa

P424

Schafer, Matthew .............................................................................. P807

Schafler, Tiferet ............................................................................................... P98

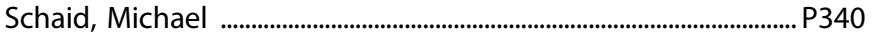

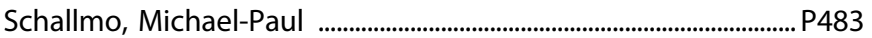

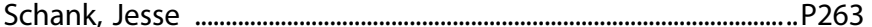

Schappi, Jeffrey ................................................................................... P369

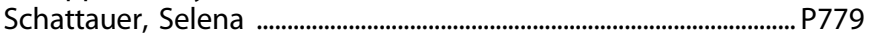

Schatzberg, Alan .............................................................................. P22

Scheinost, Dustin ................................................................................. P593

Schettler, Pamela ................................................................................... P361

Schiavon, Emanuele ............................................................................... P87

Schieber, Jennifer ................................................................................ P612

Schifani, Christin …m.... P497

Schiller, Crystal ............................................................................... 53.3, P351

Schiller, Daniela ........................................................................................... P807

Schindler, Abigail ................................................................................................... P45

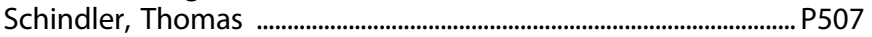

Schlessinger, Avner ................................................................................P120

Schmaal, Lianne ........................................................................................... P364

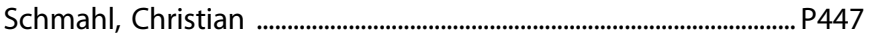

Schmidt, Mathias V.

Schmidt, Peter ................................................... 10.4, P107, P147, P351

Schmitt, Lauren ......................................................................P173, P428

Schmitt, William

P606, P631

Schmohe, Kennedy .............................................................................P126

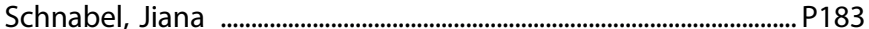

Schneck, Christopher ................................................................................. P341

Schneck, Noam .................................................................................... P448

Schneider, Bernard ............................................................................... P75

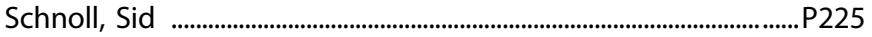

Schoenbaum, Geoffrey ........................................................................ P52

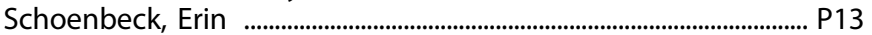

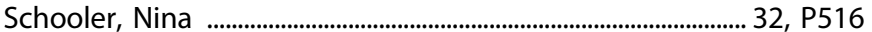

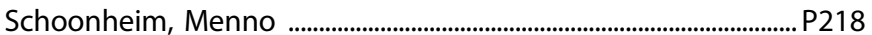

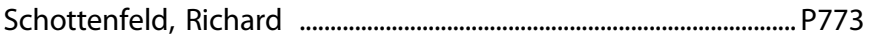

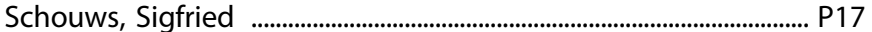

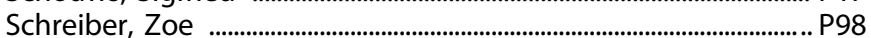

Schretlen, David A. .............................................................................P487

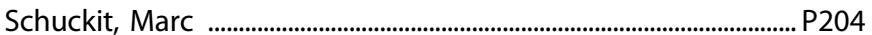

Schuler, Heike .................................................................................................. P91

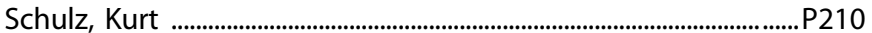

Schulze, Jurgen ....................................................................................... P562

Schulze, Thomas .......................................................................................... P381

Schumer, Maya C. ...................................................................................P304

Schuster, Randi ........................................................................................ P606

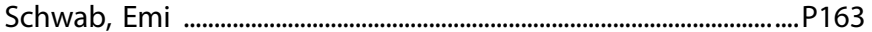

Schwandt, Melanie .................................. P285, P678, P680, P687, P691, P700, P726, P741

Schwarcz, Robert 6, .....................P572

Schwartz, Julie ............................................................................................... P805

Schweitzer, Paul ..................................................................................... P677

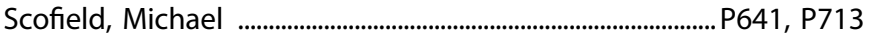

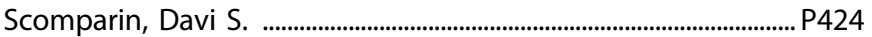

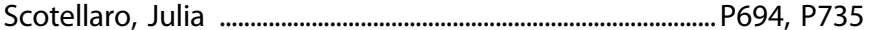

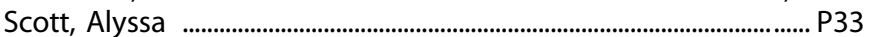

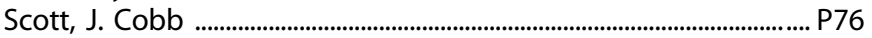

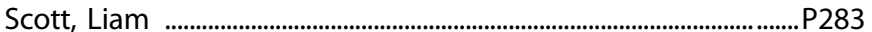

Scott, Madeline ................................................................................................... P5

Scuderi, Caterina ...................................................................................... P87

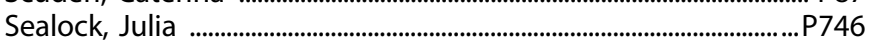

Seaman, Lauren ....................................................................................... P695

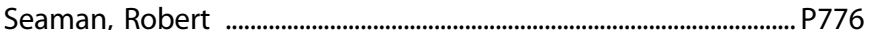

Seanez, Leann .......................................................................................... P529

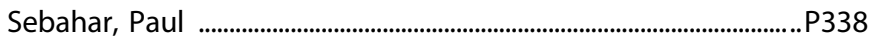

Seboek Kinter, Dalma .............................................................P581, P582

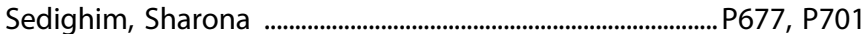

Seguin, Jean .................................................................................................P153

Sehatpour, Pejman ................................................................ P525, P574

Seidling, Kailyn .......................................................................................... P615

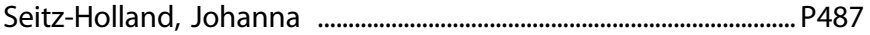

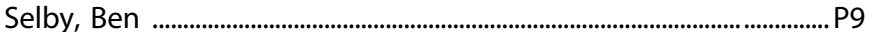

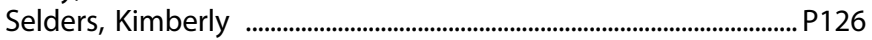

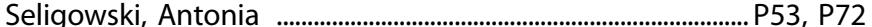

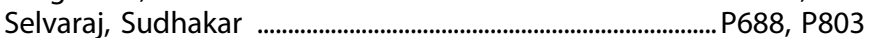

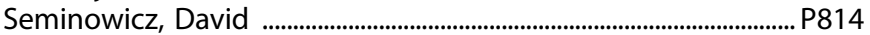

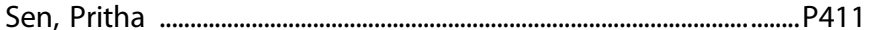

Sen, Srijan ..................................................................................................P336

Seney, Marianne ...................................... P360, P370, P532, P739, P765

Seo, Dongju ................................................................................................P593

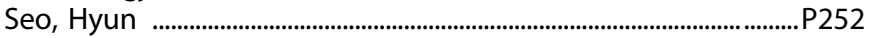

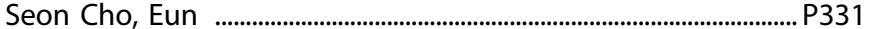

Seonjoo, Lee ...........................................................................................................P7

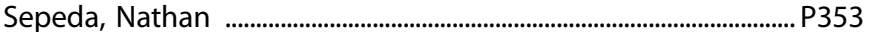

Sepulveda-Orengo, Marian .......................................................................... P656

Sequeira, Michelle ......................................................................................... P622

Serafine, Katherine .......................................................................P192

Serafini, Randal .................................................................................. P419

Sergey, Naumenko .................................................................................... P95

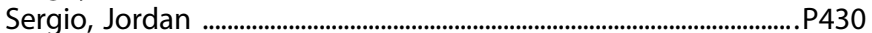

Serlin, Michael ............................................................................................ P773

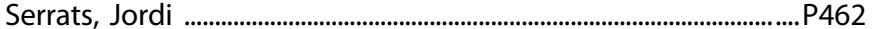

Serretti, Alessandro .................................................................................... P240

Sershen, Henry ............................................................................................. P755

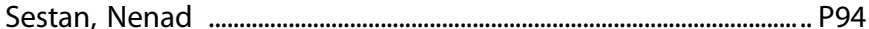

Setlow, Barry .................................................................................P410, P640

Seyfried, Nick ............................................................................................. P93

Shabalin, Andrey ..................................................................... P8 819

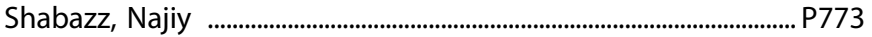

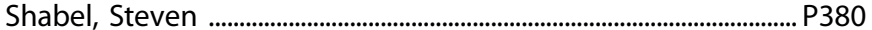

Shaffer, Rebecca ……………………………………………………….... P173

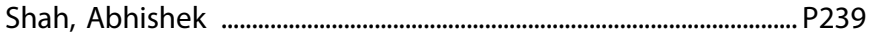

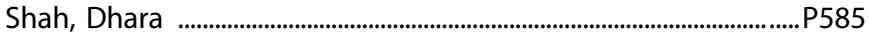

Shaham, Yavin .............................................................................................. P661

Shaikh, Jasmine ..................................................................................P342

Shankar, Kokila ............................................................................................... P677

Shankar, Vaishnavi .............................................................................P532

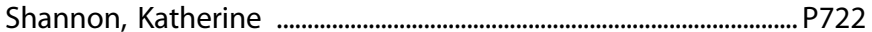

Shannon Weickert, Cynthia .....................................................................52.1

Shansky, Rebecca ......................................................................................... 58

Shanur, Sharmin ...............................................................................................P3

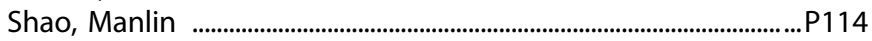

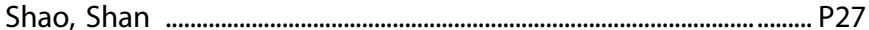

Shapiro, Daniel ......................................................................................... P55

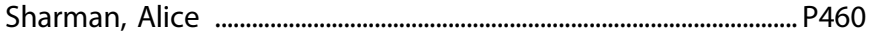

Shashkova, Leeza .......................................................................... P608

Shavitt, Roseli ............................................................................... P440, P444

Shaw, Bennett M. ................................................................................... P411

Shaw, Gladys .................................................................................................. P23

Shaw, Philip .............................................................................................P799

Shekhar, Anantha ………………………………………………......P311

Shekunov, Julia ............................................................................................ P144

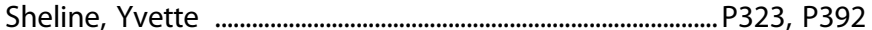

Shelton, Richard ......................................................................................... P371

Shen, Amanda .................................................................................... P511

Shen, Li ........................................................P221, P352, P417, P808

Shen, Xilin ...........................................................................................P291

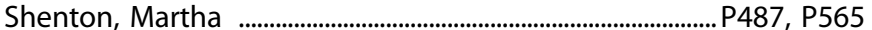

Sherafat, Yasmine ..................................................................................... P623

Sheth, Chandni ........................................................................................... P338

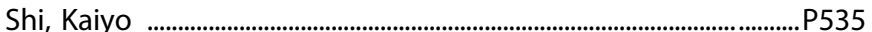

Shi, Tracey .............................................................................................P822 
Shi, Xianfeng

Shih, Pei-an (Betty)

Shim, Ruth

12,59

Shimp, Kristy P640

Shin, Joo Heon

Shin, Kuramochi

Shinn, Ann

Shinners, Grace

Shinohara, Russell

Shinohara, Taki

Shiroma, Paulo

Shoji, Hirotaka

Shokri Kojori, Ehsan

Shores, Ryan ....

Short, Annabel

Shram, Megan

Shuart, Noah G.

Shukla, Mamta

Shumann, Cynthia

Shyam, Rishab

Sial, Omar

Sibahi, Ahmad

Sibille, Etienne

Sibley, David

Siciliano, Cody

Siddarth, Prabha

Siddle, Katherine

Sideris, Beth

Sidoli, Simone

Siebels, Aubrey

Siegel, Joshua

Siemsen, Ben

Sigvard, Anne

Sikich, Linmarie

Silva, Gabriella

Silva, Ivaldo .

Silveira, Patricia

Silveri, Marisa

Sim, Fraser

Simandl, Gregory

Simerly, Richard

Simmonite, Molly

Simmons, Leigh Ann

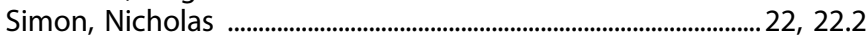

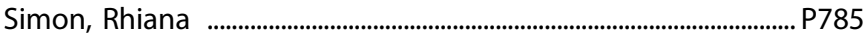

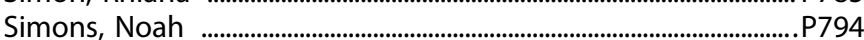

Simpson, Helen Blair ................................................................. P54, P433

Simpson, Jamie .................................................................................. P415

Simpson, Sierra .............................................................................................. P677

Simpson, Tracy ...................................................................................... P777

Singh, Aditya K. ..................................................................................P308

Singh, Archana ..................................................................................... P594

Singh, Balwinder ..................................................................... P302, P381

Singh, Jaskaran ............................................................................................ P560

Singh, Manpreet ...............................................................145, P288, P393

Singh, Praveena …………………………………………………...... P45

Singh, Raghunath ........................................................2453, P509, P559

Sinha, Rajita .................................................................................. P593, P805

Sisler, Shawna .............................................................................................P281

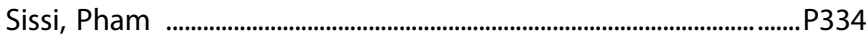

Sissoko, Cheick ................................................................................................. P264

Skarica, Mario ...................................................................................................... P94

Skime, Michelle ................................................................................. P349

Skinner, Joseph ................................................................................................... P505

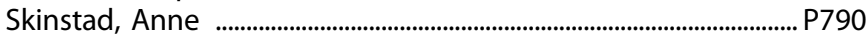

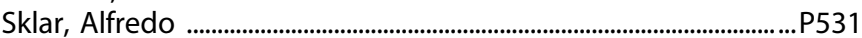

Slate, Sarah Rose ......................................................................................... P385
Slevin, Roisin ..............................................................................................P99

Slifstein, Mark .............................................................................................P210

Sloand, Timothy .................................................................................... P724

Slosky, Lauren ..............................................................................................P596

Slusher, Barbara .................................................................................... P590

Smail, Marissa ..............................................................................................P300

Smaragdi, Areti ..................................................................................... P156

Smith, Alexander ……........................................................ P194, P601

Smith, Alicia ................................................................................................. P18

Smith, Ann C. M. ................................................................................ P123

Smith, Ashley ......................................................................................... P636, P761

Smith, Caroline 44.3, .......................................................................... P129

Smith, Desmond ........................................................................ P714

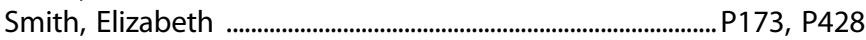

Smith, Emily ................................................................................................P453

Smith, Emma ...............................................................................................P178

Smith, Gwenn .......................................................................................P353

Smith, Janice .................................................................................P154

Smith, Kayla ...................................................................................................P748

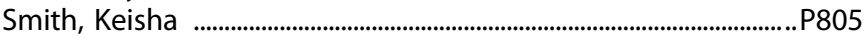

Smith, Kirsten ..................................................................................................P667

Smith, Lauren .........................................................................................P677

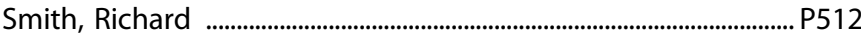

Smith, Robert ........................................................................................... P75

Smith, Ryan ...............................................................................................P719

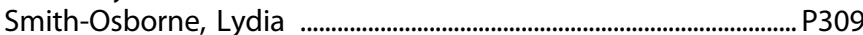

Smoski, Moria

Smucny, Jason

P388, P789

Smyser, Christopher P168

Smyser, Tara .................................................................................................P168

Smyth, Joshua M. ......................................................................................... P386

Sneider, Jennifer .......................................................................................... P169

Snijders, Clara .................................................................................... P91, P93

Snowden, Mark B. ........................................................................................... P386

Snyder, Abraham ..................................................................................... P796

Soares, Jair ......................................................P255, P275, P362, P803

Sobeih, Tarek ............................................................................................P525

Sodhi, Monsheel ....................................................................................... P466

Sofuoglu, Mehmet ............................................................................... P660

Sogani, Shivangi ............................................................................................... P70

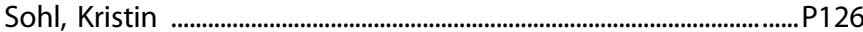

Sojourner, Kevin ...................................................................................... P83

Solberg Woods, Leah .......................................................P677, P701, P705

Soleimani, Ghazaleh ............................................................................... P652

Solingapuram Sai, Kiran Kumar ...............................................................P716

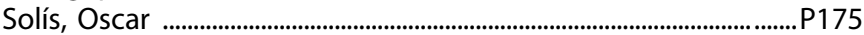

Solomon, Issac H. .............................................................................................. P4 1

Solomon, Matthew .................................................................................. P263

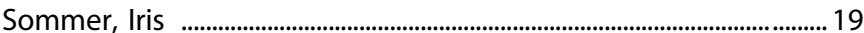

Song, Minryung ........................................................................................ P754

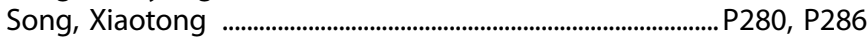

Sonmez, Ayse Irem .................................................................................... P144

Sonnenschein, Susan F. .......................................................................... P5 P58

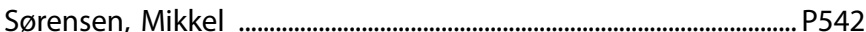

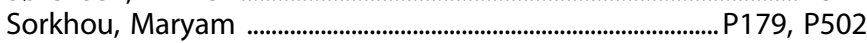

Sortman, Bo .............................................................................................P721

Souaiaia, Tade .............................................................................................. P78

Soudry, Olivia R. ................................................................................... P539

Souery, Daniel ....................................................................................... P240

Spann, Marisa ............................................................................. 49, 59, P149

Sparpana, Allison .....................................................................P280, P286

Spechler, Phillip .............................................................................................. P287

Spell, Laura ........................................................................................ P119, P203

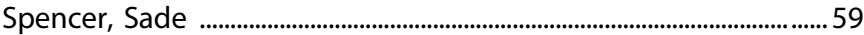

Spevack, Eli ......................................................................................................... 331

Spicer, Julie ....................................................................................................P178

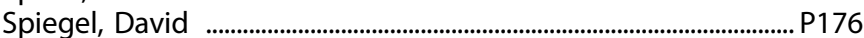

Spies, Marie ................................................................................................ P2 
Spiro, Caleb

P312

Sponheim, Scott

Sprock, Joyce

P483, P511, P564

Squassante, Lisa

P25, P573, P827

... 154

Squeglia, Lindsay

P734

Sripada, Chandra

P801

Sripathy, Srinidhi Rao

P539

Sriram, Balaji

P331

Stahl, Sarah ..

P11

Staner, Corinne

Stanford, Arielle

Stangl, Bethany

Stankovic, Srdjan

Stanley, Barbara

Stanwicks, Lauren

Starski, Phillip

Stedman, Mary

Steeby, Clara

Steele, Vaughn ....

Stegmayer, Katharina

Stein, Elliot A.

Stein, Murray

Steiner, Alex

Steiner, Johann

Steiner, Michel ...

Steinglass, Joanna

Stellman, Gabriel

Stenson, Anais

Stensson, Niclas

Stepanova, Ekaterina

Stephan, Clifford

Stephen, Julia

Stephen, Kish

Stertz, Laura

Stetz, Patrick

Stevens, Beth

Stevens, Hanna

Stevens, Jennifer

Stevens, Michael

Stewart, Brent

Stewart, Jennifer

Stewart, Kent

Stichter, Janine

Stickgold, Robert

Stiffler, Richelle

Stinley, Mark

Stirner, Madita

Stolz, Joshua M.

Stone, Lena

Strakowski, Stephen

Straub, Richard E.

Strawn, Jeffrey

Streicher, Caroline

Streit, Eleanor ..

Strickland, Justin

Stringfield, Sierra

Strober, Michael

Ströhle, Andreas

Strumila, Robertas

Strzelecki, Ashley

Stuber, Garret

Stutz, Sonja ...

Styner, Martin

Su, Graham

Subbie, Stacey

Subotnik, Kenneth

Subramaniam, Punitha

Subramanian, Aruna

.. P584

P196

P517

P110

P145, P287, P719

.P590

P126

P522

..P270, P304

P467

...P288, P332
Suen, Paulo

P803

Suett, Mark

P541

Suko, Azra

P600

Sullivan, Elizabeth

P280, P286

Sullivan, Kyle ...............................................................................................P180

Sullivan, Liam ..................................................................................................P805

Summerfelt, Ann .....................................................................................P514

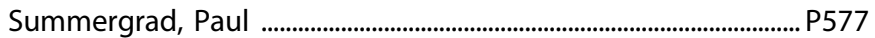

Sun, Zhifu ................................................................................................P375

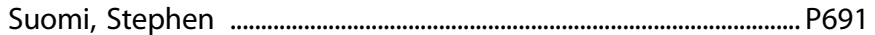

Suppes, Trisha …………………………………………………………….... P386

Suppo, Jude ................................................................................................P739

Sutherland, Ashley ....................................................................................... P17

Suzuki, Mikio ............................................................................................P208

Suzuki, Takakuni ......................................................................................... P436

Svenningson, Per ....................................................................................... P400

Svikis, Dace ....................................................................................................P583

Swain, James ………………………….....................................................P690

Swan, Blyss ...............................................................................................P678

Swann, Alan .....................................................................................................P328

Swartzwelder, H. Scott .......................................................................P774

Sweeney, John

Sweet, Robert

P173, P428, P487, P503

Sweis, Brian

P449, P468, P536, P549

Swerdlow, Neal

Sydnor, Valerie

Sylvester, Chad

Szabo, Steven T.

P342

Szatmari, Peter

Szeszko, Philip

P25, P476, P562, P573, P576

P28

P517

P418

Szumlinski, Karen

Szymkowicz, Sarah ................................................................................... P368

Tabuteau, Herriot ....................................................................... P245, P246

Taffe, Michael ................................................................................... 40, P589

Takahashi, Kazushi ..................................................................................... P426

Takahashi, Nicole ...................................................................................P126

Takeuchi, Hiroyoshi ............................................................................... P390

Talati, Ardesheer .................................................................................... P299

Talledo, Jo

Tallman, Maxwell

P25, P476, P562, P576

P397, P398, P399

Talmage, David

Tamminga, Carol

$P 4, P 256$

Tamouza, Ryad

P380, P487, P572

Tan, Chunfeng . P380

Tan, Shimin .

P768

Tan, Shuping

P522

Tan, Thomas

Tan, Vinh

P232, P469, P497

Tan, Yunfei .............P405

Tanaka-Koshiyama, Kumiko ................................................................... P552

Tang, Amy ...................................................................................................P336

Tang, Sunny ...................................................................................................P496

Tang, Wei ..

Tangmose, Karen

P441

Tapersa, Tinashe

$P 526, P 542$

Tapert, Susan

$P 148, P 214$

Tapia, Cynthia M. P308

Tardif, Christine .............................................................................................. P10

Targum, Steven ..................................................................................... P369

Taschbach, Felix H. ...................................................................................... P27

Tasker, Jeffrey .............................................................................................. P89

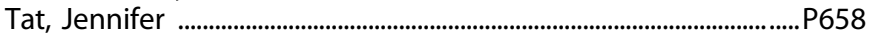

Taylor, S. Trevor ..................................................................................... P505

Taylor, Samuel ................................................................................ P719

Taylor, Stephan ……………………………………........P391, P436, P520

Taylor, Warren .......................................................................................... P368

Taylor-Yeremeeva, Elisa ...................................................................... P35

Taylor-Zapata, Perdita . . . . . . . . . . . . . . . . . 5 
Teague, Collin

Teboul, Eric

P207

Teferi, Marta

Tegang, Karly

P323

Teicher, Martin

...P56

Teichman, Emily

P389

Teixeira, Antonio

P177

Tejeda, Hugo

P688, P803

Teklehaimanot, Abeba ................................................................................ P406

Telli, Olufunmilayo ..................................................................................... P37

Tennen, Howard ................................................................................................ P6

tenOever, Benjamin .................................................................................. P419

Tentor, Zachary ....................................................................................... P557

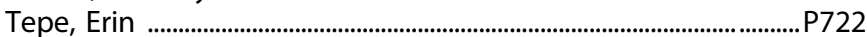

Terjo, Leonardo .......................................................................................... P311

Terranova, Joseph ........................................................................................ P528

Terrell-Hamilton, Flora .........................................................................P773

Terry, Garth ..........................................................................................P777

Tervo-Clemmens, Brenden ........................................................................... P606

Tessari, Michela ..................................................................................... P584

Thai, Michelle ...............................................................................................P817

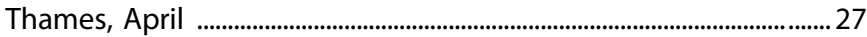

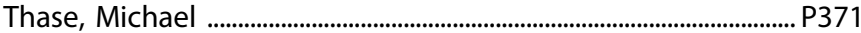

Theriault, Rachel-Karson .......................................................................... P356

Therriault, Joseph ..................................................................................... P10

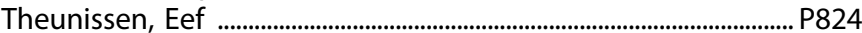

Thibeault, Kimberly .......................................................2629, P658, P743

Thomas, Craig J. ..................................................................................... P233

Thomas, Jennifer ...................................................................................... P186

Thomas, Michael ....................................................................................... P573

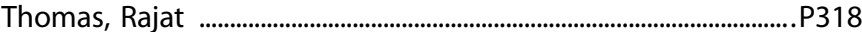

Thomas, Ronald G. ..................................................................... P99, P777

Thomas, Shruthi .................................................................................. P612

Thomas, Zachariah ...................................................................................... P246

Thomason, Moriah .............................................................................23, 23.3

Thompson, Andrew ........................................................... P95, P195

Thompson, Bob ………………………………………………………….... P300

Thompson, lan ........................................................................................... P12

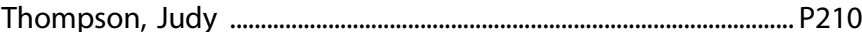

Thompson, Scott ………………............................................15, P598

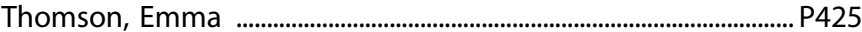

Throesch, Benjamin .................................................................................. P4 P4

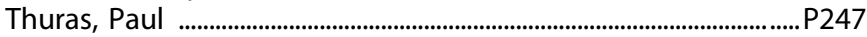

Thurman, Louise .................................................................................. P28

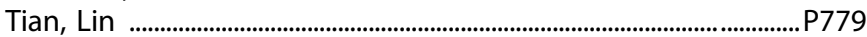

Tian, Lucy ...............................................................................................P507

Tian, Marc ...................................................................................................P541

Tian, Ruoyu ...........................................................................................P798

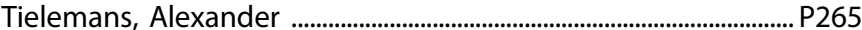

Tieman, Melanie ............................................................................................P8

Tieu, Lani ......................................................................................P677, P701

Tiihonen, Jari ............................................................................................... 19

Tillman, Rebecca ......................................................................................... P135

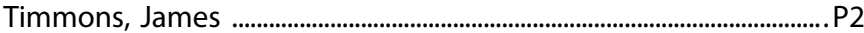

Tippani, Madhavi ..................................................................................... P5

Tippler, Maria ................................................................................................P153

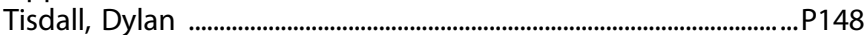

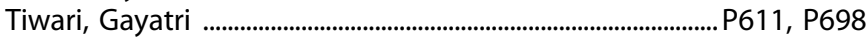

Tobe, Russel ........................................................................................P154

Toeroek, Michael ............................................................................................ P584

Tofani, Solange ....................................................................................... P657

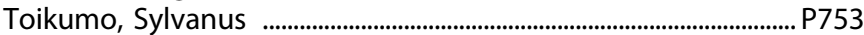

Toivainen, Sanne _........................................................................................P711

Tolliver, Bryan …………………….......................................................P307

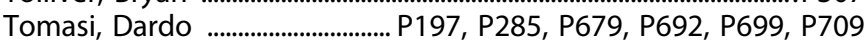

Tomie, Arthur ..............................................................................................P757

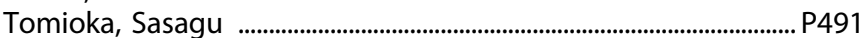

Tondo, Lucca Pizzato ....................................................................................... P675
Toney, Glenn ............................................................................................. P69

Tong, Junchao .............................................................................................. P767

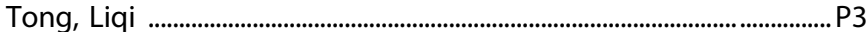

Toor, Usman .....................................................................................................P757

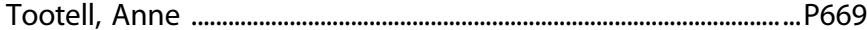

Tootell, Roger ................................................................................................P816

Toro-Serey, Claudio .................................................................................. P441

Torregrossa, Mary .......................................................2620, P643, P739

Torrence, Natasha ................................................................................... P531

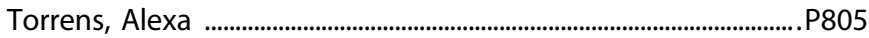

Torres, Katy ……………………………………………………………....... P38

Torres-Berrio, Angelica .............................................................P301, P730

Torres-Berrío, Angélica ........................................................... 16.2, P221

Torres-Carmona, Edgardo ................................................................. P579

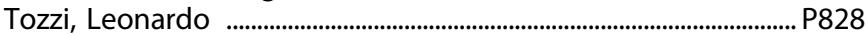

Trambaiolli, Lucas ...................................................................................... P441

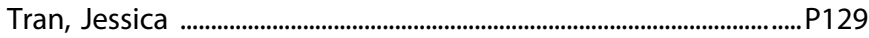

Tran, Matthew Nguyen ........................................................................P539

Trapp, Nicholas ..................................................................................... P321

Traversa, Sergio .................................................................................... P315

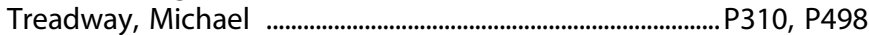

Tremblay, Marie-Eve ................................................................................ P114

Tremblay, Richard E. ……………......................................................P153

Tretyak, Valeria ........................................................................................ P332

Trevaskis, Natalie ................................................................................. P415

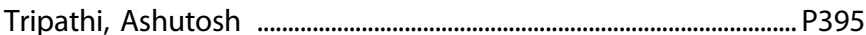

Tripi, Jordan .................................................................................................P705

Trivedi, Madhukar ................................... 47, P1, P20, P241, P349, P394

Trombetta, Bianca A. ............................................................................ P411

Tromp, Do .............................................................................................. P85

Troyer, Emily A. .......................................................................................... P29

Truby, Natalie ...........................................................................................P723

Trujillo Pisanty, Ivan ...........................................................................P785

Truong, Jennifer ............................................................................................P767

Truong, Peter ...........................................................................................P579

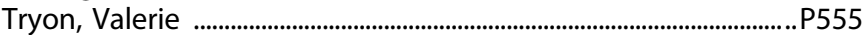

Tsai, Shangying ............................................................................................ P17

Tsang, Erica .................................................................................................P481

Tsen, Chieh-En J. ............................................................................. P388

Tseng, Angela ...........................................................................................P150

Tseng, George ........................................................................ P532

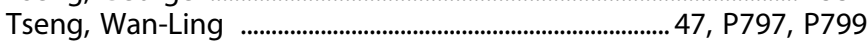

Tso, Ivy ...................................................................................................P436

Tsuang, Ming T .................................................................................... P545

Tsugawa, Sakiko ......................................................................................... P579

Tsvetkov, Evgeny …………………………………............................P712

Tuesta, Luis ..............................................................................................P624

Tully, Laura M .......................................................................................P555

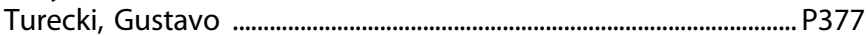

Turetsky, Bruce .................................................................................................. P495

Turkson, Susie ............................................................................................. P170

Turna, Jasmine .............................................................................................. P440

Turner, Cortney ............................................................................................ P336

Turner, Makeda ................................................................................. P598

Tuscher, Jennifer .......................................................................................... P689

Tyburski, Matthew ...................................................................................P728

Tye, Kay

39, 39.2, P27, P267, P672

P247, P302

Tyrka, Audrey ............................................................................. P44

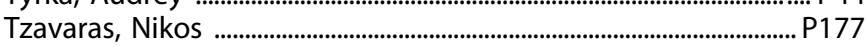

Uchida, Hiroyuki ..................................................................................... P390

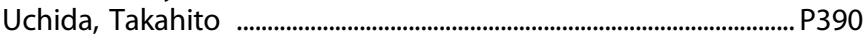

Ueno, Fumihiko ............................................................................................. P5 P59

Uher, Rudolf ....................................................................................................P363

Uhl, George .................................................................................................P782

Uliana, Daniela .............................................................................. 109, P268

Umbricht, Daniel ......................................................................... P460

Umpierrez, Guillermo ................................................................... P48, P72 
Underhill, Suzanne .................................................................................P619

Underwood, Mark ....................................................................................... P264

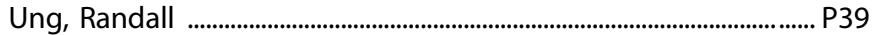

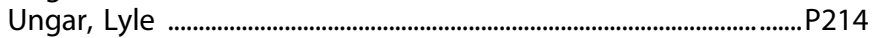

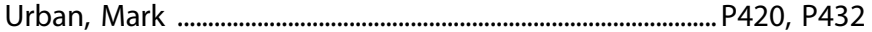

Ursini, Gianluca .............................................................................. 28.3, P558

Uthayathas, Subramaniam ................................................................ P188

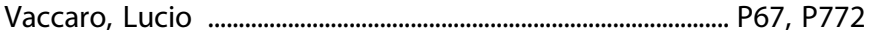

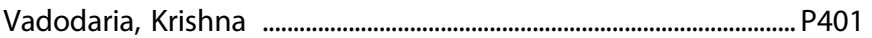

Vaiana, Alexandra .......................................................................................... P12

Vaino, Andrew ......................................................................................P507

Valcarcel, Alessandra .............................................................................P148

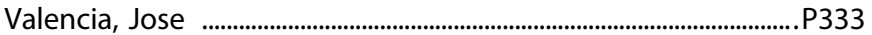

Valenta-Schnidler, Ines .................................................................... P507

Valientes, David ................................................................................... P108

Valkonen-Korhonen, Minna ....................................................................P215

Van Ameringen, Michael ....................................................................P440

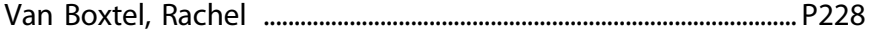

van der Merwe, Celia .........................................................................P124

Van Der Water, Judy .................................................................P165, P543

Van der Zee, Yentl ......................................................P221, P301, P708

van Dijk, Milenna ..................................................................................P299

Van Eck, Kathryn ...........................................................2200, P202, P238

van Harmelen, Anne-Laura .................................................................P364

Van Meter, Anna .................................................................................P133

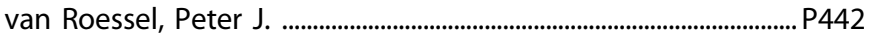

van Rooij, Sanne .............................................................. P79, P788

van Velzen, Laura ..................................................................................P364

van Wingen, Guido ..................................................................................... P318

Vande Voort, Jennifer ...........................................................................P144

Vandekar, Simon ............................................................................................... P148

Vandeloo, Katie ................................................................................................. P376

Vangel, Mark .............................................................................................P565

Vannan, Annika .......................................................................................P634

Vanover, Kimberly ..................................................................................P209

VanRyzin, Jonathan .................................................................P117, P814

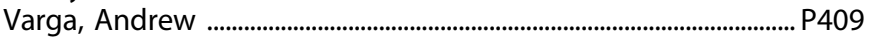

Varney, Mark ...............................................................................................P420

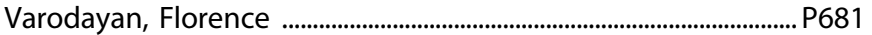

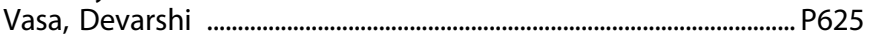

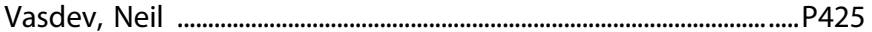

Vassileva, Jasmin .......................................................................................... P693

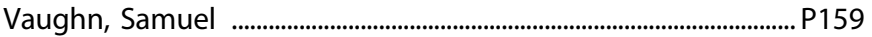

Vazquez, Andres ..............................................................................P745

Vazquez, Gustavo ................................................................................... P382

Veenstra-Vanderweele, Jeremy ……………………………....P154, P446

Vega, Jennifer ............................................................................................P368

Vega-Reyes, Adrianisse ........................................................................... P73

Veizaj, Elvana ......................................................................................P415

Velarde, Nathan ........................................................................................ P677

Velazquez-Hernandez, Geronimo ....................................................... P39

Veldic, Marin ...............................................................P302, P375, P381

Vendruscolo, Leandro .........................................................................P731

Venkareddy, Lalith K. .........................................................................P747

Venkatesh, Shyamala .................................................................................... P686

Venkatsan, Sridevi ............................................................................P431

Venniro, Marco ......................................................................................... P661

Ventriglia, Emilya .................................................................................... P175

Ventura, Joseph .......................................................................................P515

Venuti, Lauren ........................................................................................ P163

Verdoorn, Hannah ................................................................................. P605

Vernon, Anthony ................................................................................................. P463

Verplaetse, Terril ................................................................................................ P58

Versace, Amelia …………………………………................ P284, P304

Versavel, Stacey .......................................................................................... P493

Vickers-Smith, Rachel ............................................................................ P753

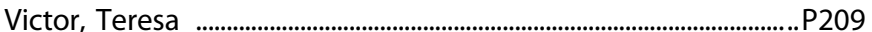

Vieira, Erica

P248, P354
Vieta, Eduard ............................................................................................. P17

Viher, Petra Verena ...................................................................................P487

Vijay, Aishwarya .................................................................................. P766

Vilca, Samara ................................................................................................P624

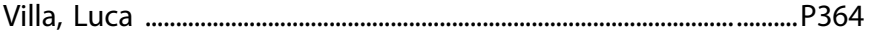

Villarin, Joseph ................................................................................................P207

Villarreal, Jannely .........................................................................................P151

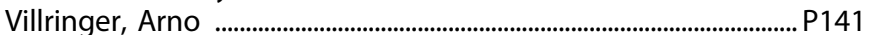

Vinkers, Christiaan ............................................................................ P218, P318

Vinogradov, Sophia ....................................................................................... 40

Vinson, Tonette .............................................................................................. P687

Viola, Thiago Wendt .................................................................P134, P675

Vismara, Matteo E.M. ....................................................................P440

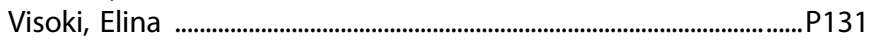

Vitaliano, Gordana ......................................................................................... P389

Vitaro, Frank .............................................................................................P153

Vitela, Melissa ..............................................................................................P671

Vitulano, Lawrence ............................................................................P146

Vivas Miranda, José Garcia ..............................................................P384

Vladut Talor, Monica ................................................................................P569

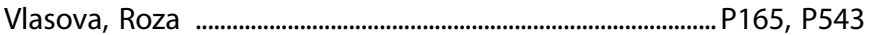

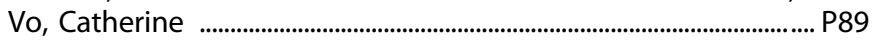

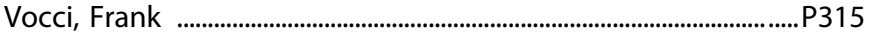

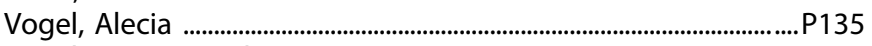

Vogelgsang, Jonathan ......................................................................... P236

Voineskos, Aristotle ............................37.2, P9, P314, P469, P487, P497

Voineskos, Daphne ................................................................................ P232

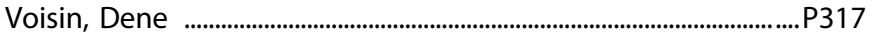

Volkow, Nora ............................ 7, P197, P285, P679, P692, P699, P709

Vollenweider, Franz ...................................................................................... 2.3

Volmar, Claude-Henry ................................................................................. P2

Volmer, Kelsey ............................................................................................... P713

Voloudakis, Georgios .........................................................................P575

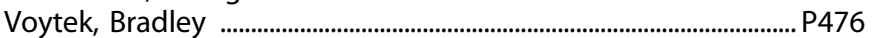

Vutukuri, Rlthvik .............................................................................................. P89

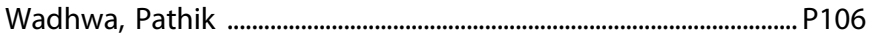

Wadsworth, Ann ...................................................................................... P308

Wadsworth, Paul A. ....................................................................................P308

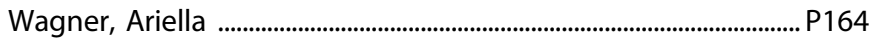

Wagner, Florence F. ............................................................................... P472

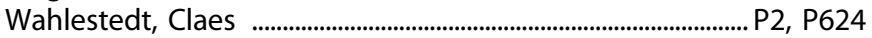

Wakeford, Alison ............................................................................................ P317

Wakschlag, Lauren ......................................................................................... 47

Waldman, Irwin ................................................................................................. P703

Walker, Deena .............................................................................. 10.1, P294

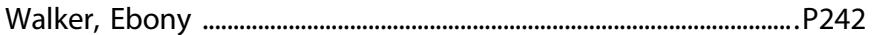

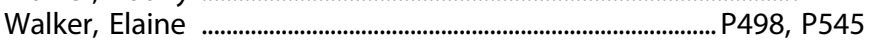

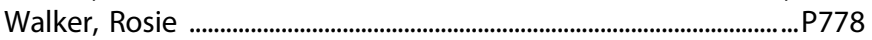

Waller, Cameron ................................................................................... P762

Walling, David ................................................................................................. P493

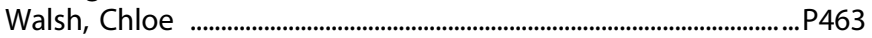

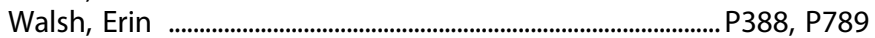

Walsh, Jessica ..........................................................................................P177

Walsh, Margaret ............................................................................................ P156

Walsh, Sheila ..................................................................P678, P680, P700

Walss-Bass, Consuelo ....................................................P358, P362, P688

Walter, Jordan .......................................................................................... P815

Walters, Brandon ..................................................................................... P49

Walther, Donna ........................................................................................P732

Walther, Sebastian ........................................................................................ P487

Wandel, Christoph .................................................................................. P154

Wang, Changning ...................................................................................... P422

Wang, Gene-Jack ...................................... P197, P679, P692, P699, P709

Wang, Hee-Hwan ..................................................................................... P132

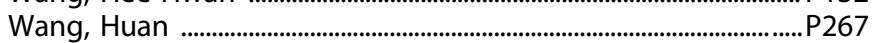

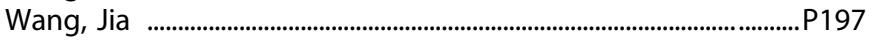

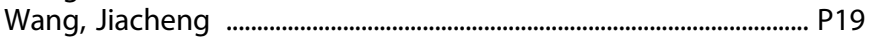

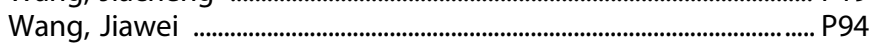


Wang, Jun

P352, P522

Wang, Junying

.. P404

Wang, Lei

Wang, Liewei

P472, P522, P791

Wang, Pingyuan

P349

Wang, Rong

P308, P563

Wang, Szu-Yung (Ariel)

P768

Wang, Wei

P692

Wang, Weiwei

P314, P497, P782

.P647

Wang, Wengang

... P13

Wang, Wen-Tung

P699, P709

Wang, Xinchen ............................................................................................ P566

Wang, Xingmei ........................................................................................... P554

Wang, Xingming ..................................................................................... P405

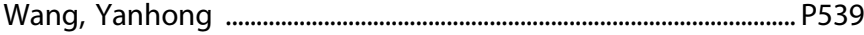

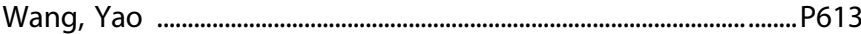

Wang, Yunwanbin ...............................................................................P594

Wang, Zhe ...............................................................................................P522

Wang, Zhoheng ...........................................................................................P171

Wang, Zi-Jun ...............................................................................................P594

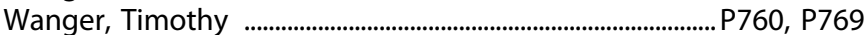

Ward, Heather .......................................................................................... P475

Ward, Sara ............................................................................................... P26

Warner, Barbara ........................................................................................ P168

Warren, Brandon ....................................................................................P721

Warsh, Jerry ..................................................................................P767

Washington, Kamryn ............................................................................... P345

Watson, Kathleen ......................................................................................... P22

Watson Jr., Stanley ......................................................................................... P336

Watson Lin, Katie ............................................................................. P312

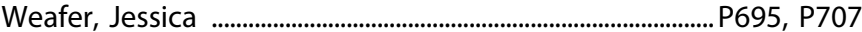

Wearick, Luis Eduardo ...............................................................................P134

Weaver, Darian ........................................................................................ P678

Webb, Bradley ............................................................................................P226

Webb, Lauren ................................................................................................P375

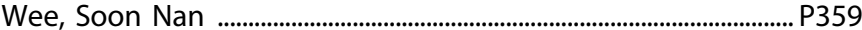

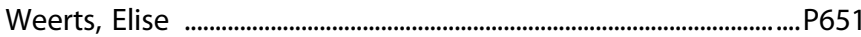

Wegener, Amy ......................................................................................... P23

Wei, Haichao ...............P688

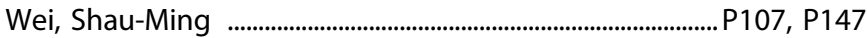

Weickert, Thomas …………………………………………........52, 52.3

Weinberg, Lauren ......................................................................................... P336

Weinberger, Daniel ............................................................. 28, P521, P539

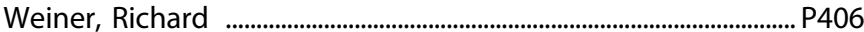

Weinschutz Mendes, Hellen ..................................................................P171

Weinshenker, David ...........................................................................P437

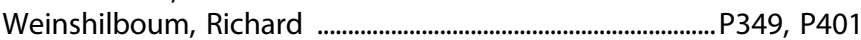

Weintraub, Sandra .................................................................................P791

Weiser, Mark ............................................................13, 13.2, 19, P544

Weisler, Rlchard ...................................................................................... P28

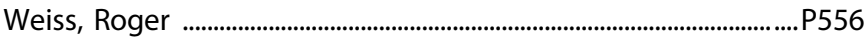

Weiss, Tamina Leonie .............................................................................. P711

Weissman, Myrna .......................................................................P149, P299

Weisstaub, Noelia .................................................................... P802

Weïwer, Michel .................................................................................................. P4

Wellek, Stefan ……………………….........................................................P350

Wels, Joseph .................................................................................................P247

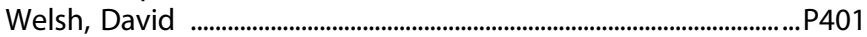

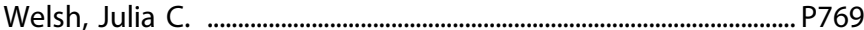

Wen, Zhenfu ........................................................................................... P76, P92

Wenderoth, Nicole .............................................................................P137

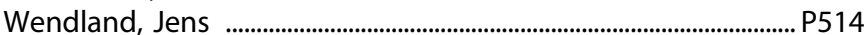

Wengler, Kenneth .....................................................................P404, P473

Wennerholm, Michelle ......................................................................P423

Wesley, Michael ................................................................................................. P707

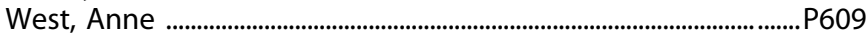

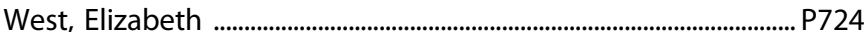

West, Jef ..................................................................................................P456
Westlund Schreiner, Melinda ............................................................P115

Westphal, Annaka ................................................................................P713

Wetherell, Julie .............................................................................. P354

Wetherill, Leah .............................................................................................. P184

Wetherill, Reagan ................................................................................... P692

Wetsel, William ...........................................................................P547, P596

Whalen, Diana ............................................................................................... P135

Whalley, Heather ......................................................................................... P778

Wheaton, Michael ........................................................................................ P54

Wheelan, Sarah ........................................................................................ P558

Wheeler, Grace .................................................................................................. P606

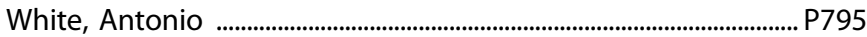

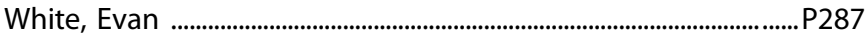

White, Lauren ..............................................................................................P495

Whitehead, Carl .............................................................................................. P395

Whiteley, Laura ................................................................................................... P2 206

Whitfield-Gabrieli, Susan .....................................................................P467

Whiting, Kimberly .................................................................................... P263

Whye, Alicia ..............................................................................................................P7

Wichmann, Juergen ................................................................................... P56

Wichmann, Romy ......................................................................................... P267

Wiedmeyer, Brandi .................................................................................... P673

Wiers, Corinde …………………………………..........2285, P679, P692

Wig, Philip ...................................................................................P420, P432

Wilkerson, Jr., Johnny .......................................................................... P790

Wilkinson, Samuel T. .................................................................................. P271

Williams, Alexia ........................................................................................ P294

Williams, Jamal .............................................................................. P612

Williams, Leanne ......................................... 37.1, P253, P386, P442, P828

Williams, Lisa .................................................................................................. P85

Williams, Mariah ............................................................................................... P26

Williams, Nolan .............................................................................176, P811

Williams, Rapheal ................................................................................... P644

Williams, Tammy ............................................................................................... P777

Williford, Kellie ................................................................................................. P86

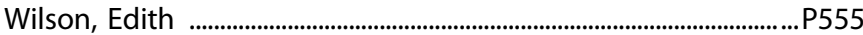

Wilson, Kathleen ......................................................................................... P29

Wilson, Kayla ................................................................................................P786

Wilson, Sean D. ................................................................................................. P517

Wilton, Angelina ........................................................................................ P349

Wimmer, Mathieu ..................................................P26, P294, P684, P737

Winder, Danny .................................................................... P86, P599, P601

Wingfield, Kelly ............................................................................................. P331

Wingo, Aliza ............................................................................................ P93

Wingo, Thomas ................................................................................................... P93

Winham, Stacey ............................................................P375, P381, P762

Winstanley, Catharine .....................................................................................4. 46.4

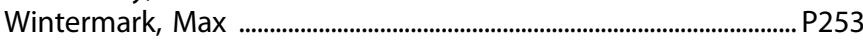

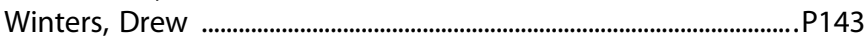

Wiseman, Jennifer ........................................................................................ P461

Wiskerke, Joost ........................................................................................ P36

Wisniewski, Thomas ..................................................................................... P409

Withey, Sarah ............................................................................................P653

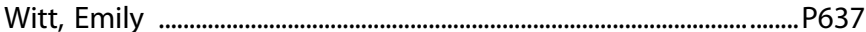

Wittmann, Marion ........................................................................................... P235, P283

Witwer, Kenneth .................................................................................... P830

Wojcik, Joanne D. ............................................................................... P487

Wojtas, Ireneusz .....................................................................................P607

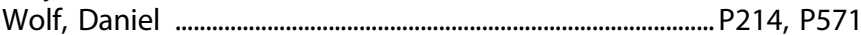

Wolfe, Sarah ...............................................................................................P681

Wolff, Max ...........................................................................................P350

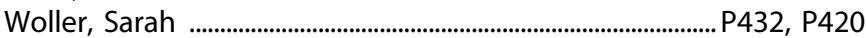

Wong, Brenden ................................................................................................ P7 P

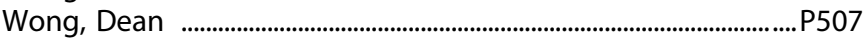

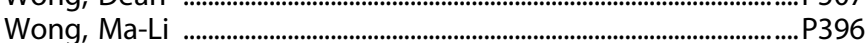

Wong, Wan-wa ....................................................................................... P49

Woo, Junsung …………………………………………………………...P358

Wood, Andrea .............................................................................................. P556 
Wood, Elizabeth

P691

Wood, Marcelo

Wood, Samuel

Woodcock, Eric

Woodhouse, Kristen

Woodruff, Grace

Woods, Scott W.

Woodside, Blake

Woodward, Neil

Woolley, Josh ...

Woolwine, Bobbi

Worster, Brooke

Wright, Clinton B.

Wright, Michael

Wright, Rachael

Wu, Jiaqian

$\mathrm{Wu}$, Mon-Ju

Wu, Qiong

Wu, Sally

$\mathrm{Wu}$, Weimiao

$\mathrm{Wu}$, Xiaojun

Wu, Xizi

Wu, Yue

Wulff, Andreas

Wyatt, Ryan

Xia, Cedric Huchuan

Xia, Naomi

Xiao, Jingyuan

Xiao, Lan

Xiao, Yang

Xie, Yang

Xiong, Qiaojie

$\mathrm{Xu}$, Cynthia

Xu, Ellie

$X u$, Heng

$\mathrm{Xu}$, Junqian

$\mathrm{Xu}, \mathrm{Li}$

$X u$, Ting

$\mathrm{Xu}$, Xiangmin

Xu, Xiaoyan

$\mathrm{Xu}$, Zhun

Xue, Xianging

Yala, Joy

Yamada, Mei

Yamamoto, Bryan

Yamamoto, Jun

Yan, Rongzhen

Yan, Zhen

Yang, Hyerim

Yang, Jie

Yang, Jun

Yang, Lingling

Yang, Minsu

Yang, Yunting

Yao, Emily

Yao, Wei-Dong

Yasmin, Farzana

Yates, John

Yatomi, Taisuke

Ye, Fengdan

Ye, Zengyou

Yeh, Hung-wen

Yeh, Szu-ying

Yehuda, Rachel

Yeisley, Jenna

Yen, William

Yepes, Beatriz

Yesavage, Jerome
....P3

P712

P592

P612

P330, P423

P545

P189

P486

.P465

P310

P633

P429

P739

P122

P688

P803

P814

P509, P559

P171

P322

..P688

P562

P598

P617

P148, P214

..P213

P797

P386

..P264

P594

P535

P425

P403, P799

P753

P752

P711

P137

P387

P210

P555

P370

P17

..P553

34.4

P528

P613

16.1

P725

P404

P189

P472

P754

P400

P694

P744

P342

P591

P390

P749

..P539

P209

..P657

2, P62

P207

P331

P172

P253
Yip, Sarah

P749

Yocum, Anastasia

P765

Yohn, Samantha

P510

Yokell, Daniel

P500

Yokose, Jun

P528

Yonga, Michele-Vera

Yorgason, Jordan

Yost, Jonathan

Young, Andrea

Young, Dmitri

Young, Jared

Young, Keith

Young, Kymberly

P679, P699, P709

P366

P200, P202, P238

Young, Larry

Young, Robert

Youngstrom, Eric

... P42

$\mathrm{Yu}$, Eunah

24, 42.4, P815

Yu, Lei .

Yu, Nam-Kyung

$\mathrm{Yu}$, Qiongru

Yu, Xiao-man

... P94

Yuan, Kai

Yuan, Qiaoping

43.2

Yue, Shuwen

Yueh, Hannah

Yun, Sujin

Yurgelun-Todd, Deborah

Yuruk, Deniz

Yushmanov, Victor

Yusofova, Lyubov

Zabik, Nicole

Zachariou, Venetia

Zachry, Jennifer

Zai, Gwyneth

Zakiniaeiz, Yasmin

Zammit, Gary

Zanao, Tamires

Zand Vakili, Amin

Zandi, Peter

Zandvakili, Amin

Zanos, Panos

Zapetis, Sarah

Zapp, Christian

Zarate, Carlos

Zarate Jr., Carlos A.

P112, P252

P406

P160, P200, P202, P238

P472

P757

P591

.. P28

P522

... P699, P709

P138

..P594

P446

P617

P34, P237, P281

P144

P284

P216

P59

Zatorski, Nicole

Zavala, Arturo

Zeak, Jennifer

Zell, Vivien

Zelli, Danielle

Zeni, Courtney

Zerbi, Valerio

Zeto, Amy

Zetterberg, Henrik

Zhang, Fan

Zhang, Feng

Zhang, Huaibo

Zhang, Huaiyu

Zhang, Jason

Zhang, Jiahe

Zhang, Jian

Zhang, Jing

Zhang, Rui ..

Zhang, Sheng

Zhang, Wenchao

Zhang, Xiaolu

Zhang, Xue

Zhang, Yan-Ling
P417, P419, P730

P655

P146

P766

P582

$\mathrm{P} 803$

P250

$\mathrm{P} 276$

P384

P233, P327

P816

P799

40, P240, P402

P233

P120

P704

P739

P330

P254

P491

P137

.P535

P306

P565

P267

P747

..P42

P129

P467

P184

P94

.P285, P692, P699, P709

P780

P197

P559

P253, P386, P828

P472 
Zhang, Yi

Zhao, Hongyu

Zhao, Joan

Zhao, Zhongming

P355, P688

Zharovsky, Elizabeth .............................................................................. P461

Zheng, Dan ...........................................................................................P415

Zheng, JunQi .............................................................................................P750

Zheng, MingQiang ................................................................................ P668

Zhou, Charles ...............................................................................................P337

Zhou, Chengwen ....................................................................................P529

Zhou, Hang ..................................................................................... P753, P819

Zhou, Jia .................................................................................... P308, P563

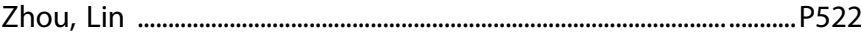

Zhou, Pengcheng ....................................................................................... P39

Zhou, Zhenhe ........................................................................................P522

Zhu, J. Julius ....................................................................................30, 30.4

Zhu, Wei ...............................................................................................P522

Zhu, Ziyu ................................................................................... P397, P398

Ziausyte, Ugne .................................................................................................. P687

Ziegler, Thomas ............................................................................................. P361

Zilverstand, Anna ............................................................................................. P595

Zimering, Jeffrey ...................................................................................P419

Zimmermann, Jan ................................................................................ P277

Zipunnikov, Vadim ..................................................................................P121

Zoghbi, Anthony ........................................................................... 48.1, P566

Zohar, Joseph ...........................................................................................P240

Zomorrodi, Reza .................................................................... P232, P314

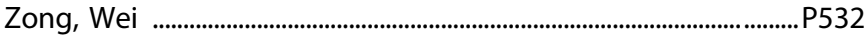

Zonshayn, David ...................................................................................... P98

Zopfs, Malte .................................................................................................P447

Zorrilla, Eric ...........................................................................................................46.3

Zou, Kai ..................................................................................................P522

Zsido, Rachel G ....................................................................................P141

Zuardi, Antonio W. ................................................................................P424

Zubieta, Jon-Kar ................................................................................... P819

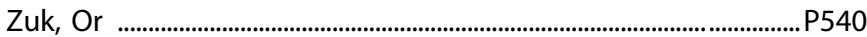

Zuo, Yantao ..............................................................................................P716

Zurcher, Nicole ........................................................................ 16.3, P388

Zwicker, Alyson ......................................................................................... P363 